\title{
Aspects of high scale leptogenesis with low-energy leptonic CP violation
}

\author{
A. Granelli, ${ }^{a}$ K. Moffat ${ }^{b}$ and S. T. Petcov ${ }^{a, c, d}$ \\ ${ }^{a} S I S S A / I N F N$, \\ Via Bonomea 265, 34136 Trieste, Italy \\ ${ }^{b}$ Institute for Particle Physics Phenomenology, Department of Physics, Durham University, \\ South Road, Durham DH1 3LE, U.K. \\ ${ }^{c}$ Kavli IPMU (WPI), University of Tokyo, \\ 5-1-5 Kashiwanoha, 277-8583 Kashiwa, Japan \\ ${ }^{d}$ Institute of Nuclear Research and Nuclear Energy, Bulgarian Academy of Sciences, \\ 1784 Sofia, Bulgaria \\ E-mail: agranell@sissa.it, kristianpascalmoffat@gmail.com, \\ petcov@sissa.it
}

ABSTRACT: Using the density matrix equations (DME) for high scale leptogenesis based on the type I seesaw mechanism, in which the $\mathrm{CP}$ violation (CPV) is provided by the low-energy Dirac or/and Majorana phases of the neutrino mixing (PMNS) matrix, we investigate the 1-to-2 and the 2-to-3 flavour regime transitions, where the 1, 2 and 3 leptogenesis flavour regimes in the generation of the baryon asymmetry of the Universe $\eta_{B}$ are described by the Boltzmann equations. Concentrating on the 1-to-2 flavour transition we determine the general conditions under which $\eta_{B}$ goes through zero and changes sign in the transition. Analysing in detail the behaviour of $\eta_{B}$ in the transition in the case of two heavy Majorana neutrinos $N_{1,2}$ with hierarchical masses, $M_{1} \ll M_{2}$, we find, in particular, that i) the Boltzmann equations in many cases fail to describe correctly the generation of $\eta_{B}$ in the 1, 2 and 3 flavour regimes, ii) the 2-flavour regime can persist above (below) $\sim 10^{12} \mathrm{GeV}\left(\sim 10^{9} \mathrm{GeV}\right)$, iii) the flavour effects in leptogenesis persist beyond the typically considered maximal for these effects leptogenesis scale of $10^{12} \mathrm{GeV}$. We further determine the minimal scale $M_{1 \text { min }}$ at which we can have successful leptogenesis when the CPV is provided only by the Dirac or Majorana phases of the PMNS matrix as well as the ranges of scales and values of the phases for having successful leptogenesis. We show, in particular, that when the CPV is due to the Dirac phase $\delta$, there is a direct relation between the sign of $\sin \delta$ and the sign of $\eta_{B}$ in the regions of viable leptogenesis in the case of normal hierarchical light neutrino mass spectrum; for the inverted hierarchical spectrum the same result holds for $M_{1} \lesssim 10^{13} \mathrm{GeV}$. The considered different scenarios of leptogenesis are testable and falsifiable in low-energy neutrino experiments.

Keywords: Neutrino Physics, Cosmology of Theories beyond the SM, CP violation ARXIV EPRINT: 2107.02079 


\section{Contents}

1 Introduction 1

2 The framework $\quad 4$

2.1 Neutrino masses and neutrino (lepton) mixing 4

$\begin{array}{ll}2.2 & \text { The seesaw mechanism }\end{array}$

2.3 The baryon asymmetry of the universe and flavoured leptogenesis 9

3 The baryon asymmetry sign change $\quad 15$

$\begin{array}{lll}3.1 & \text { Strong wash-out regime } & 18\end{array}$

$\begin{array}{ll}3.2 \text { Weak wash-out regime } & 19\end{array}$

$\begin{array}{lll}3.2 .1 & \text { Vanishing initial abundance } & 19\end{array}$

$\begin{array}{ll}3.2 .2 & \text { Thermal initial abundance } \\ \end{array}$

3.3 Transitions between different flavour regimes: detailed analysis 20

4 The case of decoupled $N_{3}$

4.1 CP violation from low-energy CPV phases of the PMNS matrix 24

$\begin{array}{lll}4.2 & \mathrm{CP} \text { violation due to the Dirac phase } & 27\end{array}$

4.2.1 The case of real $R$-matrix $(x \neq 0$ and $y=0) \quad 28$

4.2.2 Purely imaginary $R_{11} R_{12}\left(R_{12} R_{13}\right)(x=k \pi, k=0,1,2, y \neq 0)$

$\begin{array}{lll}\text { 4.3 } & \mathrm{CP} \text { violation due to the Majorana phases } & 37\end{array}$

5 Summary and conclusions $\quad 44$

$\begin{array}{lll}\text { A DMEs from the three- to the two-flavour basis } & 47\end{array}$

B Approximated solutions to the BEs in various regimes $\quad 50$

B.1 Strong wash-out regime 51

B.2 Weak wash-out regime 51

B.2.1 Vanishing initial abundance $\quad 51$

B.2.2 Thermal initial abundance $\quad 52$

C Approximate 1-to-2 flavour transitional mass scale 53

\section{Introduction}

In spite of the fact that the leptogenesis idea of the origin of the matter-antimatter, or baryon, asymmetry of the Universe is 35 years old [1,2], the leptogenesis scenario of the asymmetry generation continues to be actively investigated (see, e.g., the recent review article [3] which includes also an extended list of references). A very attractive version 
of leptogenesis is that based on type I seesaw mechanism of neutrino mass generation [48], which also corresponds to the original scenario proposed in [1]. The type I seesaw mechanism provides a natural explanation of the smallness of neutrino masses and via leptogenesis establishes a link between the existence and smallness of neutrino masses and the existence of the baryon asymmetry. A basic ingredient of the seesaw scenario are the singlet RH neutrinos $\nu_{l R}$ (singlet RH neutrino fields $\nu_{l R}(x)$ ), by which the Standard Model (SM) can be extended without modifying its fundamental properties. Such an extension with two RH neutrinos is the minimal set-up in which leptogenesis can take place, satisfying the three Sakharov conditions [9] for a dynamical generation of the baryon asymmetry. In leptogenesis the requirement of lepton charge non-conservation is satisfied, as is well known, due to a Majorana mass term of the RH neutrinos $\nu_{l R}$ and the Yukawa coupling $\mathcal{L}_{\mathrm{Y}}(x)$ of $\nu_{l R}$ with the Standard Model lepton and Higgs doublets, $\psi_{l L}(x)$ and $\Phi(x)$, while the requisite $\mathrm{C}$ - and $\mathrm{CP}$-symmetry violations are ensured by the $\nu_{l R}$ Majorana mass term and/or the Yukawa coupling $\mathcal{L}_{\mathrm{Y}}(x)$. Both the $\nu_{l R}$ Majorana mass term and $\mathcal{L}_{\mathrm{Y}}(x)$ respect the $\mathrm{SU}(2)_{L} \times \mathrm{U}(1)_{Y_{W}}$ symmetry of the SM. In the diagonal mass basis of the RH neutrinos $\nu_{l R}$ and the charged leptons $l^{ \pm}, l=e, \mu, \tau, \mathcal{L}_{\mathrm{Y}}(x)$ and the Majorana mass term are given by:

$$
\mathcal{L}_{\mathrm{Y}, \mathrm{M}}(x)=-\left(Y_{l i} \overline{\psi_{l L}}(x) i \tau_{2} \Phi^{*}(x) N_{i R}(x)+\text { h.c. }\right)-\frac{1}{2} M_{i} \overline{N_{i}}(x) N_{i}(x),
$$

where $Y_{l i}$ is the matrix of neutrino Yukawa couplings (in the chosen basis) and $N_{i}\left(N_{i}(x)\right)$ is the heavy Majorana neutrino (field) possessing a mass $M_{i}>0$.

In the present article we revisit the high (GUT) scale flavoured leptogenesis scenario, which is realised for masses of the heavy Majorana neutrinos in the range $M_{i} \sim\left(10^{9}-\right.$ $\left.10^{14}\right) \mathrm{GeV}$, i.e., for $M_{i}$ by a few to several orders of magnitude smaller than the unification scale of electroweak and strong interactions, $M_{G U T} \cong 2 \times 10^{16} \mathrm{GeV}$. The values of the masses $M_{i}$, i.e., their scale and spectrum, set the scale of leptogenesis. In this scenario, the out-of-equilibrium decays $N_{j} \rightarrow l^{+}+\Phi^{(-)}$and $N_{j} \rightarrow l^{-}+\Phi^{(+)}$of the heavy Majorana neutrinos $N_{j}$, caused by the CP non-conserving neutrino Yukawa couplings in eq. (1.1), proceed with different rates, producing $\mathrm{CP}$ violating (CPV) asymmetries in the flavour lepton charges $L_{l}, l=e, \mu, \tau$, and in the integral lepton charge $L=L_{e}+L_{\mu}+L_{\tau}$, of the Universe. The lepton asymmetries thus generated are converted into a baryon asymmetry by $(B-L)$-conserving, but $(B+L)$-violating, sphaleron processes which exist in the SM and are effective at temperatures $T \sim\left(132-10^{12}\right) \mathrm{GeV}$.

For heavy Majorana neutrinos $N_{1,2,3}$ with hierarchical mass spectrum, $M_{1} \ll M_{2} \ll$ $M_{3}$, there exists, as is well known, a lower bound on the mass of the lightest $N_{1}$ for which the matter-antimatter asymmetry can be generated in leptogenesis: $M_{1} \gtrsim 10^{9} \mathrm{GeV}$ [10]. Furthermore, when flavour effects in leptogenesis [11-13] (see also [14-16]) are taken into account, leptogenesis was shown to be possible at scales compatible with the quoted lower bound with the requisite $\mathrm{CP}$ violation provided exclusively by the Dirac and/or Majorana phases in the Pontecorvo, Maki, Nakagawa, Sakata (PMNS) neutrino (lepton) mixing matrix $U_{\text {PMNS }}$ [17-24]. Rather detailed studies performed in 2018 in [25, 26] have shown that in the case of spectrum of masses of the heavy Majorana neutrinos with mild hierarchy, $M_{2} \sim 3 M_{1}, M_{3} \sim 3 M_{2}$, the "flavoured" leptogenesis can successfully generate the observed 
baryon asymmetry at scales as low as $\sim 10^{6} \mathrm{GeV}$, i.e., for $M_{1} \gtrsim 10^{6} \mathrm{GeV}$, and that in this case as well the Dirac or Majorana CPV phases present in the PMNS matrix can be the unique source of the required $\mathrm{CP}$ violation. Moreover, in [27] the same conclusion concerning the Dirac or Majorana phases being the source of CP violation in leptogenesis, was shown to hold in the case of the so-called "Neutrino Option" seesaw scenario [28] in which the term quadratic in the Higgs field in the Higgs potential, responsible for the breaking of the SM electroweak symmetry, is generated radiatively at one loop by the neutrino Yukawa coupling in eq. (2.5).

The studies performed in $[25,26]$ were based on the density matrix equations (DME) for leptogenesis [29-31], in which, in particular, the flavour decoherence effects associated with the charged lepton Yukawa couplings are accounted for in the various regimes they go through continuously (from negligible to non-negligible but non-thermalised to fully thermalised) in the expanding and cooling Universe. In contrast, the Boltzmann equations (BE) describe flavour effects in leptogenesis only for either negligible or fully thermalised charged lepton Yukawas. As a consequence, the DME approach to leptogenesis opened up the possibility to investigate quantitatively the behaviour of the baryon asymmetry $\eta_{B}$ in transitions between the different flavour regimes in leptogenesis. The analysis done in [26] revealed that in the case of heavy Majorana neutrinos with hierarchical mass spectrum, $M_{1} \ll M_{2} \ll M_{3}$, the baryon asymmetry $\eta_{B}$ can go through zero, changing sign at certain scale $M_{1}$ in the transition between the unflavoured (or single flavoured) and the two flavoured leptogenesis regimes, associated with the $\tau$-Yukawa coupling. Moreover, when the $\mathrm{CP}$ violation is provided in leptogenesis by the low-energy CPV phases present in the PMNS matrix, the width of the transition was shown to become extremely large and to lead to the existence of a "plateau" in the baryon asymmetry dependence on the scale $M_{1}$.

In the present article we continue to investigate the transitions between the different flavour regimes in high scale leptogenesis based on type I seesaw mechanism with hierarchical heavy Majorana neutrinos, $M_{1} \ll M_{2} \ll M_{3}$, began in [26]. We consider the case in which the $\mathrm{CP}$ violation (CPV) in leptogenesis is provided by the low-energy Dirac or/and Majorana phases of the PMNS neutrino mixing matrix. Using the density matrix equations (DME) to describe high scale leptogenesis, we investigate in detail the 1-to-2 and the 2-to-3 flavour regime transitions, where the 1, 2 and 3 leptogenesis flavour regimes in the generation of the baryon asymmetry $\eta_{B}$ are described by the Boltzmann equations. Concentrating on the 1-to-2 flavour transition we determine the general conditions under which $\eta_{B}$ goes through zero and changes sign in the transition and $\left|\eta_{B}\right|$ reaches a plateau as $M_{1}$ increases. We analyse further in detail the behaviour of $\eta_{B}$ in the transition under these conditions in the case of two heavy Majorana neutrinos $N_{1,2}$ with hierarchical masses, $M_{1} \ll M_{2}$, and identify, in particular, cases in which the baryon asymmetry exhibits a "non-standard" behaviour in the transition. We determine the minimal scale $M_{1 \text { min }}$ as well as the corresponding ranges of $M_{1}$ and of the Dirac and Majorana CPV phases for which we can have successful leptogenesis when the requisite $\mathrm{CP}$ violation in leptogenesis is provided only either by the Dirac or by the Majorana phases of the PMNS matrix.

The paper is organised as follows. In section 2 we summarise the existing data on the neutrino masses and mixing that we use in our analysis, the basics of the type I 
seesaw scenario, and introduce the 1, 2 and 3 flavour Boltzmann equations as well as the density matrix equations that are employed in our study, elucidating the role of the charged lepton Yukawa couplings. In section 3 we investigate the baryon asymmetry $\left(\eta_{B}\right)$ sign change in the transition between the one and two flavour regimes in the case of three heavy Majorana neutrinos $N_{1,2,3}$ with hierarchical masses, $M_{1} \ll M_{2} \ll M_{3}, M_{1} \gtrsim$ $10^{9} \mathrm{GeV}$, determine the general conditions under which the sign change takes place and then perform a detailed analysis of the transitions between the different flavour regimes. To make the discussion as transparent as possible we investigate in section 4 the behaviour of the baryon asymmetry under the general conditions under which the sign change of the baryon asymmetry $\eta_{B}$ in the 1-to-2 flavour regimes is possible in the case of decoupled $N_{3}$, in which the number of parameters is significantly smaller than in the general case with three heavy Majorana neutrinos. The thorough analysis is performed in this section with the $\mathrm{CP}$ violation necessary for the generation of the baryon asymmetry provided by the low-energy Dirac or/and Majorana phases present in the PMNS neutrino mixing matrix. We conclude in section 5 with a summary of our results.

\section{The framework}

\subsection{Neutrino masses and neutrino (lepton) mixing}

Throughout the present study we employ the reference 3-neutrino mixing scheme (see, e.g., [32]):

$$
\nu_{\alpha L}(x)=\sum_{j=1}^{3} U_{\alpha j} \nu_{j L}(x),
$$

where $\nu_{\alpha L}(x), \alpha=e, \mu, \tau$, is the left-handed (LH) flavour neutrino field (which enters into the expression of the weak interaction Lagrangian), $\nu_{j L}(x), j=1,2,3$, is the LH component of the field of a light neutrino $\nu_{j}$ with mass $m_{j}$, and $U$ is the $3 \times 3$ unitary Pontecorvo-MakiNakagawa-Sakata (PMNS) neutrino (lepton) mixing matrix. We consider the case of light massive neutrinos $\nu_{j}$ being Majorana particles and will use in our analysis the standard parametrisation of the PMNS matrix [32] in this case:

$$
U=\left(\begin{array}{ccc}
c_{12} c_{13} & s_{12} c_{13} & s_{13} \mathrm{e}^{-i \delta} \\
-s_{12} c_{23}-c_{12} s_{23} s_{13} \mathrm{e}^{i \delta} & c_{12} c_{23}-s_{12} s_{23} s_{13} \mathrm{e}^{i \delta} & s_{23} c_{13} \\
s_{12} s_{23}-c_{12} c_{23} s_{13} \mathrm{e}^{i \delta} & -c_{12} s_{23}-s_{12} c_{23} s_{13} \mathrm{e}^{i \delta} & c_{23} c_{13}
\end{array}\right) \times\left(\begin{array}{ccc}
1 & 0 & 0 \\
0 & \mathrm{e}^{\frac{i \alpha_{21}}{2}} & 0 \\
0 & 0 & \mathrm{e}^{\frac{i \alpha_{31}}{2}}
\end{array}\right) .
$$

Here $c_{i j} \equiv \cos \theta_{i j}, s_{i j} \equiv \sin \theta_{i j}$, the angles $\theta_{i j}=[0, \pi / 2], \delta=[0,2 \pi)$ is the Dirac CP violation (CPV) phase, and $\alpha_{21}$ and $\alpha_{31}$ are the two Majorana CPV phases [33], $\alpha_{21(31)}=[0,4 \pi]{ }^{1}$ The Dirac and Majorana phases can be sources of low-energy leptonic CP violation. In the case of CP invariance, we have $\delta=0, \pi$ and $\alpha_{21(31)}=k_{2(3) 1} \pi, k_{2(3) 1}=0,1,2,3,4$.

In what concerns the light neutrinos masses $m_{1,2,3}$, we use the "standard" convention of numbering the neutrino mass eigenstates in which $\Delta m_{21}^{2} \equiv m_{2}^{2}-m_{1}^{2}>0$ and $\Delta m_{31(32)}^{2} \equiv$

\footnotetext{
${ }^{1}$ Within the type I seesaw mechanism of neutrino mass generation we will consider the mass-eigenstate neutrinos to be Majorana fermions and it proves convenient to work with this extended range of possible values of the two Majorana phases $\alpha_{21,31}[22]$ (see further).
} 


\begin{tabular}{c|cccccc}
\hline Ordering & $\begin{array}{c}\theta_{12} \\
\left({ }^{\circ}\right)\end{array}$ & $\begin{array}{c}\theta_{13} \\
\left({ }^{\circ}\right)\end{array}$ & $\begin{array}{c}\theta_{23} \\
\left({ }^{\circ}\right)\end{array}$ & $\begin{array}{c}\delta \\
\left(^{\circ}\right)\end{array}$ & $\begin{array}{c}\Delta m_{\odot}^{2} \\
\left(10^{-5} \mathrm{eV}^{2}\right)\end{array}$ & $\begin{array}{c}\Delta m_{\mathrm{atm}}^{2} \\
\left(10^{-3} \mathrm{eV}^{2}\right)\end{array}$ \\
\hline NO & $33.44_{-0.74}^{+0.77}$ & $8.57_{-0.12}^{+0.12}$ & $49.2_{-1.2}^{+0.9}$ & $197_{-24}^{+27}$ & $7.42_{-0.20}^{+0.21}$ & $2.517_{-0.028}^{+0.026}$ \\
\hline IO & $33.45_{-0.75}^{+0.78}$ & $8.60_{-0.12}^{+0.12}$ & $49.3_{-1.1}^{+0.9}$ & $282_{-30}^{+26}$ & $7.42_{-0.20}^{+0.21}$ & $-2.498_{-0.028}^{+0.028}$ \\
\hline
\end{tabular}

Table 1. Best-fit values and $1 \sigma$ allowed ranges of the neutrino mixing angles $\theta_{12}, \theta_{13}, \theta_{23}$, and of the $\Delta m_{\odot}^{2} \equiv \Delta m_{21}^{2}$ and $\Delta m_{\text {atm }}^{2} \equiv \Delta m_{31}^{2}\left(\Delta m_{\text {atm }}^{2} \equiv \Delta m_{32}^{2}\right)$ in the case of NO (IO) light neutrino mass spectrum, obtained in [36]. We quote also the best-fit value and $1 \sigma$ allowed ranges of the Dirac CPV phase $\delta$ from [36]. However, these data on $\delta$ are not used in our analyses.

$m_{3}^{2}-m_{1(2)}^{2}$ are associated, together respectively with the angles $\theta_{12}$ and $\theta_{23}$, with the observed flavour conversion of solar (electron) neutrinos $\nu_{e}$ and the dominant oscillations of atmospheric muon neutrinos and antineutrinos, $\nu_{\mu}$ and $\bar{\nu}_{\mu}$, while the angle $\theta_{13}$, together with $\Delta m_{31(32)}^{2}$, is associated with the reactor $\bar{\nu}_{e}$ oscillations observed in the Daya Bay, RENO and Double Chooz experiments [32]. The enormous amount of neutrino oscillation data accumulated over many years of research (see, e.g., [34]) made it possible to determine $\Delta m_{21}^{2}, \sin ^{2} \theta_{12},\left|\Delta m_{31}^{2}\right|\left(\left|\Delta m_{32}^{2}\right|\right), \sin ^{2} \theta_{23}$ and $\sin ^{2} \theta_{13}$ with remarkably high precision (see, e.g., $[35,36])$. We report in table 1 the best-fit values and $1 \sigma$ ranges of the three neutrino mixing (or PMNS) angles and the two neutrino mass squared differences obtained from the global neutrino oscillation data analysis in [36]. In the numerical analyses we will perform, we will use the best-fit values of the three neutrino mixing angles and of the two neutrino mass squared differences quoted in table 1.

The existing neutrino data, as is well known, do not allow to determine the sign of $\Delta m_{31(32)}^{2}$ and the two values of $\operatorname{sgn}\left(\Delta m_{31(32)}^{2}\right)$ correspond to two possible types of light neutrino mass spectrum - with normal ordering (NO) and inverted ordering (IO), which is reflected in table 1 . In the adopted convention, the two spectra read:

- Normal Ordering (NO): $m_{1}<m_{2}<m_{3}, \Delta m_{31}^{2} \equiv \Delta m_{\text {atm }}^{2}>0$;

- Inverted Ordering (IO): $m_{3}<m_{1}<m_{2}, \Delta m_{32}^{2} \equiv \Delta m_{\text {atm }}^{2}<0$.

Depending on the value of the lightest neutrinos mass, the neutrino mass spectrum can also be:

- Normal Hierarchical (NH): $0 \simeq m_{1} \ll m_{2}<m_{3}$, with $m_{2} \simeq \sqrt{\Delta m_{21}^{2}}$ and $m_{3} \simeq \sqrt{\Delta m_{31}^{2}} ;$

- Inverted Hierarchical (IH): $0 \simeq m_{3} \ll m_{1}<m_{2}$, with $m_{1} \simeq \sqrt{\left|\Delta m_{32}^{2}\right|-\Delta m_{21}^{2}}$ and $m_{2} \simeq \sqrt{\left|\Delta m_{32}^{2}\right|} ;$

- Quasi Degenerate (QD): $m_{1} \simeq m_{2} \simeq m_{3}$, with $m_{1,2.3}^{2} \gg\left|\Delta m_{31(32)}^{2}\right|$.

All considered spectra are compatible with the existing data on the light neutrino masses [32]. The IO spectrum is disfavoured at approximately $2.7 \sigma$ C.L. with respect 
to the NO spectrum by the global neutrino oscillation data [36]. In our further analyses we will mostly be interested in the $\mathrm{NH}$ and $\mathrm{IH}$ spectra.

A few comments relevant for our further discussion are in order. As it follows from table 1, we have $\Delta m_{21}^{2} \ll\left|\Delta m_{31(32)}^{2}\right|, \Delta m_{21}^{2} /\left|\Delta m_{31(32)}^{2}\right| \cong 1 / 30$. Apart from some hints from the data of the $\mathrm{T} 2 \mathrm{~K}$ and $\mathrm{NO} \nu \mathrm{A}$ experiments $[37,38]$ that the Dirac phase $\delta \sim 3 \pi / 2$, no other experimental information on the Dirac and Majorana CPV phases in the PMNS matrix is available at present. The values of $\delta$ obtained in the global analyses $[35,36]$ have relatively large uncertainties. In view of this we will treat both the Dirac phase $\delta$ and the Majorana phases $\alpha_{21}$ and $\alpha_{31}$ as free parameters in our study. We recall that with $\theta_{13} \cong$ 0.15 , the Dirac phase $\delta$ can generate CP violating effects in neutrino oscillations $[33,39,40]$, i.e., a difference between the probabilities of the $\nu_{\alpha} \rightarrow \nu_{\beta}$ and $\bar{\nu}_{\alpha} \rightarrow \bar{\nu}_{\beta}$ oscillations, $\alpha \neq$ $\beta=e, \mu, \tau$. The magnitude of CP violation in $\nu_{\alpha} \rightarrow \nu_{\beta}$ and $\bar{\nu}_{\alpha} \rightarrow \bar{\nu}_{\beta}$ oscillations $(\alpha \neq \beta)$ is determined by [41] the rephasing invariant

$$
J_{\mathrm{CP}}=\operatorname{Im}\left(U_{\mu 3} U_{e 3}^{*} U_{e 2} U_{\mu 2}^{*}\right),
$$

which $^{2}$ in the standard parametrisation of the PMNS matrix has the form:

$$
J_{C P} \equiv \operatorname{Im}\left(U_{\mu 3} U_{e 3}^{*} U_{e 2} U_{\mu 2}^{*}\right)=\frac{1}{8} \cos \theta_{13} \sin 2 \theta_{12} \sin 2 \theta_{23} \sin 2 \theta_{13} \sin \delta .
$$

If the hints that $\delta$ has a value close to $3 \pi / 2$ are confirmed by future more precise data one would have $J_{C P} \cong-0.03$, implying that the CP violating effects in neutrino oscillations would be relatively large and observable in currently running and/or future neutrino oscillation experiments (T2K, NO $\nu \mathrm{A}$, T2HK, DUNE, see, e.g., [32, 34]).

In what concerns the Majorana CPV phases in the PMNS matrix, the flavour neutrino oscillation probabilities $P\left(\nu_{\alpha} \rightarrow \nu_{\beta}\right)$ and $P\left(\bar{\nu}_{\alpha} \rightarrow \bar{\nu}_{\beta}\right), \alpha, \beta=e, \mu, \tau$, do not depend on these phases [33, 43]. The Majorana phases can play important role, e.g., in $|\Delta L|=2$ processes like neutrinoless double beta $\left((\beta \beta)_{0 \nu^{-}}\right)$decay $(A, Z) \rightarrow(A, Z+2)+e^{-}+e^{-}, L$ being the total lepton charge, in which the Majorana nature of massive neutrinos $\nu_{i}$ manifests itself (see, e.g., refs. [44-46]).

Our interest in the Dirac and Majorana CPV phases present in the neutrino mixing matrix is stimulated also by the intriguing possibility that the Dirac phase and/or the Majorana phases in the PMNS matrix $U$ can provide the $\mathrm{CP}$ violation necessary for the generation of the observed baryon asymmetry of the Universe [17, 18]. In the present article we continue to explore this intriguing and very appealing possibility.

Finally we comment briefly on the current limits on the absolute scale of light neutrino masses (or equivalently on the lightest neutrino mass). Using the existing best lower bounds on the $(\beta \beta)_{0 \nu}$-decay half-lives of ${ }^{136} \mathrm{Xe}[47]$ and ${ }^{76} \mathrm{Ge}$ [48] one can obtain the following "conservative" upper limit on the light Majorana neutrino masses, which is in the range of the QD spectrum [49]: $m_{1,2,3} \lesssim 0.58 \mathrm{eV}$.

The most stringent upper limit on the light neutrino masses, which does not depend on the nature of massive neutrinos, was obtained in KATRIN experiment

\footnotetext{
${ }^{2}$ The $J_{\mathrm{CP}}$ factor in eq. (2.3) is analogous to the rephasing invariant associated with the Dirac CPV phase in the quark mixing matrix [42].
} 
by measuring the spectrum of electrons near the end point in ${ }^{3} \mathrm{H} \beta$-decay $[50,51]$ : $m_{1,2,3}<0.8 \mathrm{eV}$ (90\%C.L.).

The Cosmic Microwave Background (CMB) data of the WMAP and PLANCK experiments, combined with supernovae and other cosmological and astrophysical data can be used to obtain information in the form of an upper limit on the sum of neutrino masses. Depending on the model complexity and the input data used one typically finds [52] (see also [35]): $\sum_{j} m_{j}<(0.11-0.54) \mathrm{eV}(95 \% \mathrm{CL})$.

\subsection{The seesaw mechanism}

The leptogenesis we are going to discuss in the present article is based on the type I seesaw mechanism of neutrino mass generation [4-8]. This rather simple mechanism is realised, as is well known, by extending the Standard Model (SM) with $n \geq 2$ right-handed $(\mathrm{RH})$ neutrinos $\nu_{l R}\left(\mathrm{RH}\right.$ neutrino fields $\left.\nu_{l R}(x)\right)$, that are singlets under $\mathrm{SU}(2)_{L} \times \mathrm{U}(1)_{Y_{W}}$, possess a Majorana mass term and couple through a Yuakawa-type interaction to the SM lepton and Higgs doublets, $\left(\psi_{\alpha L}(x)\right)^{T}=\left(\nu_{\alpha L}^{T}(x) \quad \ell_{\alpha L}^{T}(x)\right)$, with $\alpha=e, \mu, \tau$, and $(\Phi(x))^{T}=\left(\Phi^{(+)}(x) \Phi^{(0)}(x)\right)$. The minimal type I seesaw scheme in which leptogenesis can be realised is with $n=2 \mathrm{RH}$ neutrinos. In this scenario the lightest neutrino $-\nu_{1}\left(\nu_{3}\right)$ for NO (IO) neutrino mass spectrum - is massless at tree and one-loop level. We will consider leptogenesis with both $n=3$ and $n=2 \mathrm{RH}$ neutrinos.

Without loss of generality, we work in the basis in which i) the Majorana mass matrix of RH neutrinos, $M$, is diagonal and positive, $M=\operatorname{diag}\left(M_{1}, M_{2}, M_{3}\right)$ with $M_{i}>0$, and ii) the charged lepton Yukawa couplings are flavour diagonal. In the chosen basis, the neutrino Yukawa and the RH neutrino Majorana mass terms are given by:

$$
\mathcal{L}_{\mathrm{Y}, \mathrm{M}}(x)=-\left(Y_{\alpha i} \overline{\psi_{\alpha L}}(x) i \tau_{2} \Phi^{*}(x) N_{i R}(x)+\text { h.c. }\right)-\frac{1}{2} M_{i} \overline{N_{i}}(x) N_{i}(x),
$$

where $Y_{\alpha i}$ is the matrix of the neutrino Yukawa coupling and $N_{i}(x)=N_{i R}(x)+N_{i L}^{c}(x)=$ $C\left(\overline{N_{i}}(x)\right)^{T}$, with $N_{i L}^{c}(x) \equiv C\left(\overline{N_{i R}}(x)\right)^{T}$ and $C$ being the charge-conjugation matrix. The fields $N_{1,2,3}(x)$ correspond to Majorana neutrinos $N_{1,2,3}$ with masses $M_{1,2,3}>0$ which in high scale leptogenesis can have values $M_{1,2,3} \sim\left(10^{6}-10^{14}\right) \mathrm{GeV}$, and so we will refer to $N_{1,2,3}$ further on as "heavy Majorana neutrinos" or just "heavy neutrinos". After the spontaneous breaking of the electroweak symmetry, the neutral component of the Higgs doublet acquires a non-vanishing vacuum expectation value (VEV) $v=246 \mathrm{GeV}$, generating a neutrino Dirac mass term which, together with the $N_{1,2,3}$ mass term, can be cast in the form:

$$
\mathcal{L}_{\nu}^{m}=-\frac{1}{2}\left(\overline{\nu_{\alpha L}} \overline{N_{i L}^{c}}\right)\left(\begin{array}{cc}
\mathbb{O}_{\alpha \beta} & \frac{v}{\sqrt{2}} Y_{\alpha j} \\
\frac{v}{\sqrt{2}}\left(Y^{T}\right)_{i \beta} & M_{i} \delta_{i j}
\end{array}\right)\left(\begin{array}{c}
\nu_{\beta R}^{c} \\
N_{j R}
\end{array}\right)+\text { h.c. },
$$

where $\alpha, \beta=e, \mu, \tau(i, j=1,2,3)$ and $\nu_{\beta R}^{c}(x) \equiv C\left(\overline{\nu_{\beta L}}(x)\right)^{T}$. This mass term can be diagonalised by means of the Takagi transformation, which, at leading order in the seesaw expansion parameter $\left|v Y_{\alpha i}\right| / M_{i} \ll 1$, leads to the well known expression for the tree-level light neutrino mass matrix $m_{\nu}^{\text {tree }}$

$$
\left(m_{\nu}^{\text {tree }}\right)_{\alpha \beta} \cong-\frac{v^{2}}{2} Y_{\alpha i} M_{i}^{-1}\left(Y^{T}\right)_{i \beta} .
$$


In the version of the high scale leptogenesis with heavy Majorana neutrino masses which are not hierarchical and have relatively low values, $M_{1} \sim 10^{6} \mathrm{GeV}, M_{2} \cong 3 M_{1}$, $M_{3} \cong 3 M_{2}$, the one-loop radiative correction to the light neutrino mass matrix can be non-negligible $[25,26] .{ }^{3}$ Moreover, the one-loop correction to light neutrino mass matrix has an opposite sign with respect to the tree-level contribution. This, in principle, allows for a partial cancellation between the two contributions. In certain cases the cancellation has to be fine-tuned in order to obtain neutrino masses compatible with the existing data. A rather detailed study of this fine-tuning problem was performed in [26] and we refer the reader to this article for further details.

We will be interested in high scale leptogenesis with hierarchical masses of the heavy Majorana neutrinos, $M_{1} \ll M_{2} \ll M_{3}$, in which the CP violation is provided exclusively by the Dirac and/or Majorana CPV phases present in the PMNS matrix. In this case successful leptogenesis is possible for $M_{1} \gtrsim 10^{10} \mathrm{GeV}[18,26]$. Under these conditions the one-loop contribution to the light neutrino mass matrix $m_{\nu}$, as our numerical study has shown, is sub-leading and amounts to $\sim(10 \%-20 \%)$ effects so that no fine-tuning problem is present in the scenarios considered in this study. Nevertheless, we have included the one-loop contribution in our calculations. This contribution is given by [54-56] (see also, e.g., [57]):

$$
\left(m_{\nu}^{1 \text {-loop }}\right)_{\alpha \beta}=Y_{\alpha i} \frac{M_{i}}{32 \pi^{2}}\left(\frac{\log \left(M_{i}^{2} / m_{H}^{2}\right)}{M_{i}^{2} / m_{H}^{2}-1}+3 \frac{\log \left(M_{i}^{2} / m_{Z}^{2}\right)}{M_{i}^{2} / m_{Z}^{2}-1}\right)\left(Y^{T}\right)_{i \beta},
$$

where $m_{H}=125 \mathrm{GeV}$ and $m_{Z}=91.2 \mathrm{GeV}$ are the Higgs and $\mathrm{Z}$ boson masses, respectively. The light neutrino mass matrix including the one-loop correction reads:

$$
\left(m_{\nu}\right)_{\alpha \beta} \equiv\left(m_{\nu}^{\text {tree }}+m_{\nu}^{1-\text { loop }}\right)_{\alpha \beta}=-\frac{v^{2}}{2} Y_{\alpha i} f\left(M_{i}\right)\left(Y^{T}\right)_{i \beta},
$$

with

$$
f\left(M_{i}\right) \equiv M_{i}^{-1}-\frac{M_{i}}{16 \pi^{2} v^{2}}\left(\frac{\log \left(M_{i}^{2} / m_{H}^{2}\right)}{M_{i}^{2} / m_{H}^{2}-1}+3 \frac{\log \left(M_{i}^{2} / m_{Z}^{2}\right)}{M_{i}^{2} / m_{Z}^{2}-1}\right) .
$$

It can be diagonalised as:

$$
\hat{m}_{\nu}=U^{\dagger} m_{\nu} U^{*}
$$

where $\hat{m}_{\nu} \equiv \operatorname{diag}\left(m_{1}, m_{2}, m_{3}\right)$. With $m_{\nu}$ given by eq. (2.9), the Casas-Ibarra parametrisation [58] of the neutrino Yukawa couplings takes the form [53]:

$$
Y_{\alpha j}= \pm i \frac{\sqrt{2}}{v} U_{\alpha a} \sqrt{m_{a}} R_{j a} \sqrt{f^{-1}\left(M_{j}\right)}
$$

where $R$ is a complex orthogonal matrix. In the present study we adopt the following parametrisation of the $R$-matrix:

$$
R=\left(\begin{array}{ccc}
1 & 0 & 0 \\
0 & c_{1} & s_{1} \\
0 & -s_{1} & c_{1}
\end{array}\right)\left(\begin{array}{ccc}
c_{2} & 0 & s_{2} \\
0 & 1 & 0 \\
-s_{2} & 0 & c_{2}
\end{array}\right)\left(\begin{array}{ccc}
c_{3} & s_{3} & 0 \\
-s_{3} & c_{3} & 0 \\
0 & 0 & 1
\end{array}\right),
$$

\footnotetext{
${ }^{3}$ The higher-order corrections to the light neutrino mass matrix were shown to be suppressed with respect to the tree-level and one-loop contributions [25, 53].
} 
where $c_{j} \equiv \cos \left(x_{j}+i y_{j}\right)$ and $s_{j} \equiv \sin \left(x_{j}+i y_{j}\right), x_{j}$ and $y_{j}$ being free real parameters $(j=1,2,3)$.

\subsection{The baryon asymmetry of the universe and flavoured leptogenesis}

The baryon asymmetry of the Universe (BAU) can be parametrised by the baryon-tophoton ratio

$$
\eta_{B} \equiv \frac{n_{B}-n_{\bar{B}}}{n_{\gamma}}
$$

where $n_{B}, n_{\bar{B}}$ and $n_{\gamma}$ are the number densities of baryons, anti-baryons and photons, respectively. Alternatively, it can be expressed in terms of the baryonic density parameter

$$
\Omega_{B} h^{2}=\eta_{B} \frac{m_{p} n_{\gamma}}{\rho_{c} h^{-2}} \cong \frac{\eta_{B}}{2.73 \times 10^{-8}},
$$

where $m_{p}$ is the proton mass, $\rho_{c}$ is the critical density of the Universe and $h$ is the Hubble expansion rate of the Universe $(H)$ per unit of $100(\mathrm{~km} / \mathrm{s}) / \mathrm{Mpc}$ (the numerical values of the constants are taken from [59]). The present BAU has been determined independently from the estimates of the Big Bang Nucleosynthesis (BBN) and, with higher precision, from the measurements of the Cosmic Microwave Background (CMB) anisotropies made by the Planck observatory. The following results have been reported (at 68\% C.L.) in [60, 61]: ${ }^{4}$

$$
\begin{aligned}
& \Omega_{B} h^{2}=0.02235 \pm 0.00049(\mathrm{BBN}) \\
& \Omega_{B} h^{2}=0.02242 \pm 0.00014(\mathrm{CMB})
\end{aligned}
$$

From both we obtain the best-fit value of

$$
\eta_{B} \cong 6.1 \times 10^{-10}
$$

that is going to be our reference value in the further analyses.

The generation of a matter-antimatter asymmetry in the expanding Universe can naturally be accomplished within the type I seesaw framework through thermal leptogenesis. Provided the Yukawa couplings in eq. (2.5) are CP violating, the out-of-equilibrium decays of the heavy Majorana neutrinos $N_{i}$ to leptons and Higgs doublets in the early Universe generate CPV asymmetries in the individual lepton flavour charges $L_{\alpha}$, as well as in the total lepton charge $L$. The so generated lepton asymmetry is then translated into an asymmetry in the baryon charge $B$ by the SM $(B+L)$-violating, but $(B-L)$-conserving, sphaleron processes, which are effective at temperatures $T \cong 132-10^{12} \mathrm{GeV}$.

In this work we will concentrate on the case in which the heavy neutrinos $N_{1,2,3}$ have hierarchical masses, namely $M_{1} \ll M_{2} \ll M_{3}$. In this case generically only the CPV decays of $N_{1}$ contribute to the generation of the CPV lepton asymmetry. We shall report in this section the relevant equations for one decaying heavy neutrino that will be used in our analysis.

\footnotetext{
${ }^{4}$ More precisely, the BBN estimate is taken from eq. (14) of [60], while that from CMB corresponds to the one reported in the last column of table 2 in [61].
} 
The charged lepton final states in the decays of the heavy neutrino $N_{i}, N_{i} \rightarrow \Phi^{+} \psi_{i}$ and $N_{i} \rightarrow \Phi^{-} \overline{\psi_{i}}$, are a superposition of the charged lepton flavour states, namely,

$$
\begin{aligned}
\left|\psi_{i}\right\rangle & =\sum_{\alpha=e, \mu, \tau} C_{i \alpha}\left|\psi_{\alpha}\right\rangle, \\
\left|\overline{\psi_{i}}\right\rangle & =\sum_{\alpha=e, \mu, \tau} \bar{C}_{i \alpha}^{*}\left|\overline{\psi_{\alpha}}\right\rangle,
\end{aligned}
$$

with the coefficients $C_{i \alpha}$ and $\bar{C}_{i \alpha}$ at tree-level ${ }^{5}$ given by

$$
C_{i \alpha}=\bar{C}_{i \alpha}=\frac{Y_{\alpha i}}{\sqrt{\left(Y^{\dagger} Y\right)_{i i}}}
$$

We are interested in the decays of $N_{1}, N_{1} \rightarrow \Phi^{+} \psi_{1}$ and $N_{1} \rightarrow \Phi^{-} \overline{\psi_{1}}$, so index $i$ should be replaced with 1 in eqs. (2.19) - (2.21).

If it were not for the SM charged lepton Yukawa interactions, the quantum states $\left|\psi_{1}\right\rangle$ and $\left|\overline{\psi_{1}}\right\rangle$ would be coherent superpositions of the charged lepton flavour states. However, when these interactions are in thermal equilibrium, i.e., their rates are larger than the expansion rate of the Universe, given the difference between the charged lepton Yukawa couplings, $h_{e}, h_{\mu}, h_{\tau}$, the flavour states become distinguishable and each flavour state experiences a different time-evolution - actually, it is enough for the SM $\tau$ - and $\mu$-Yukawa interactions to be in equilibrium for the three lepton flavours to be distinguishable. If the SM charged lepton Yukawa interactions are faster than the process of the heavy neutrino decay into (anti)leptons, then the coherence in $\left|\psi_{1}\right\rangle\left(\left|\overline{\psi_{1}}\right\rangle\right)$ is efficiently destroyed [30] (see, e.g., also [62]) - in this sense these are decoherence effects. The relevant processes are the interchanges between the $\mathrm{LH}$ leptons with their respective $\mathrm{RH}$ components and vice verse through scattering processes involving the Higgs doublet. By means of the optical theorem, the rates of these processes involving the tauon and the muon, $\Gamma_{\tau}$ and $\Gamma_{\mu}$, are given by the imaginary part of the $\tau, \mu$ thermal self-energy and read [25, 31] (see also, e.g., [63] and references therein): $\Gamma_{\tau, \mu} \cong 8 \times 10^{-3} h_{\tau, \mu}^{2} T$. The comparison of $\Gamma_{\tau}$ and $\Gamma_{\mu}$ with the Hubble expansion rate $H$ gives: $^{6}$

$$
\begin{aligned}
& \frac{\Gamma_{\tau}}{H} \cong \frac{M_{P}}{T} 4.85 \times 10^{-8} \cong\left(\frac{1 \mathrm{GeV}}{T}\right) 5.92 \times 10^{11}, \\
& \frac{\Gamma_{\mu}}{H} \cong \frac{M_{P}}{T} 1.72 \times 10^{-10} \cong\left(\frac{1 \mathrm{GeV}}{T}\right) 2.10 \times 10^{9},
\end{aligned}
$$

where $M_{P} \cong 1.22 \times 10^{19} \mathrm{GeV}$ is the Planck mass. At $T \gg 10^{12} \mathrm{GeV}$, the rates of the $\tau$ and $\mu$-Yukawa interactions are much smaller than the expansion rate of the Universe as

\footnotetext{
${ }^{5}$ The one-loop contributions to the $N_{i}$ decay would lead to corrections to $C_{i \alpha}$ and $\bar{C}_{i \alpha}$ (see, e.g., eqs. (24) and (25) of [31]). However, as these corrections would lead to sub-leading $\mathcal{O}\left(\epsilon^{2}\right)$ effects, we decided not to take them into account.

${ }^{6}$ The $\tau$ - and $\mu$-Yukawa couplings are given by $h_{\tau}=\sqrt{2} m_{\tau} / v \cong 1.02 \times 10^{-2}$ and $h_{\mu}=\sqrt{2} m_{\mu} / v \cong$ $6.08 \times 10^{-4}$, where $m_{\tau}$ and $m_{\mu}$ are the $\tau^{ \pm}$and $\mu^{ \pm}$masses, respectively, and $v=246 \mathrm{GeV}$. Given the smallness of the $e$-Yukawa coupling $h_{e}=\sqrt{2} m_{e} / v \cong 2.94 \times 10^{-6}, m_{e}$ being the $e^{\mp}$ mass, the $e$-Yukawa interactions come into thermal equilibrium only at $T \lesssim 10^{5} \mathrm{GeV}$, being therefore ineffective at the temperatures of interest in the present work.
} 
$\Gamma_{\tau, \mu} \ll 1$. As a consequence, the flavour states are indistinguishable and the (anti)leptons produced via the $N_{1}$ 's decay are always found in the coherent superposition defined in eq. (2.19) ((2.20)). This is the unflavoured or single-flavour regime. For $M \gg 10^{12} \mathrm{GeV}$, leptogenesis proceeds in the unflavoured regime for its entire duration and is usually studied within the single-flavour approximation, under which the $\mu$ - and $\tau$-decoherence effects are neglected. Correspondingly, this scenario is typically dubbed unflavoured or singleflavoured leptogenesis. In the single-flavour approximation, the time-evolution of the number densities of $N_{1}$ and $B-L$ charge can be described by the set of semi-classical singleflavoured Boltzmann equations (1BE1F):

$$
\begin{aligned}
\frac{d N_{N_{1}}}{d z} & =-D_{1}\left(N_{N_{1}}-N_{N_{1}}^{e q}\right), \\
\frac{d N_{B-L}}{d z} & =\epsilon^{(1)} D_{1}\left(N_{N_{1}}-N_{N_{1}}^{e q}\right)-W_{1} N_{B-L},
\end{aligned}
$$

where $z \equiv M_{1} / T$. The quantities $N_{N_{1}}$ and $N_{B-L}$ are respectively the number of heavy neutrinos $N_{1}$ and $B-L$ asymmetry in a comoving volume. In the present work the comoving volume is normalised as in [25-27] so that it contains one photon at $\mathrm{z}=0$, i.e., $N_{N_{1}}^{e q}(0)=3 / 4$. This normalisation within the Boltzmann statistics is equivalent to using $N_{N_{1}}^{e q}(z)=\frac{3}{8} z^{2} K_{2}(z)$, where $K_{n}(z), n=1,2, \ldots$, is the modified $n^{\text {th }}$ Bessel function of the second kind.

The decay parameter $D_{1}$ is given by:

$$
D_{1}(z)=\kappa_{1} z \frac{K_{1}(z)}{K_{2}(z)}
$$

where $\kappa_{1}$ is defined as the ratio between the total decay rate of $N_{1}$ at zero temperature, $\Gamma_{N_{1}}^{(0)}=\left(Y^{\dagger} Y\right)_{11} M_{1} / 8 \pi$, and the Hubble expansion rate $H$ at $z=1$. It proves convenient to write $\kappa_{1}$ in the following form:

$$
\kappa_{1}=\frac{\tilde{m}_{1}}{m_{*}}
$$

where

$$
\tilde{m}_{1} \equiv\left(Y^{\dagger} Y\right)_{11} v^{2} / 2 M_{1}, \quad m_{*} \equiv\left(8 \pi^{2} v^{2} / 3 M_{P}\right) \sqrt{g_{*} \pi / 5} \approx 10^{-3} \mathrm{eV},
$$

$g_{*}=106.75$ being the number of relativistic degrees of freedom at $z=1$. The wash-out parameter $W_{1}$ reads:

$$
W_{1}(z)=\frac{1}{2 N_{\ell}^{e q}} D_{1}(z) N_{N_{1}}^{e q}(z),
$$

where $N_{\ell}^{e q}$ is the equilibrium number density of leptons at $z=0$, which, within the adopted normalisation, is given by $N_{\ell}^{e q}=N_{N_{1}}^{e q}(0)=3 / 4 .^{7}$

Finally, the CPV-asymmetry parameter $\epsilon^{(1)}$ is given by [65-67]: ${ }^{8}$

$$
\epsilon^{(1)}=\frac{3}{16 \pi\left(Y^{\dagger} Y\right)_{11}} \sum_{j \neq 1} \Im\left[\left(Y^{\dagger} Y\right)_{1 j}^{2}\right] \frac{\xi\left(x_{j}\right)}{\sqrt{x_{j}}},
$$

\footnotetext{
${ }^{7}$ A detailed derivation of eqs. (2.26) - (2.29) is given, e.g., in [64].

${ }^{8}$ We work with the same sign convention used in [26], so the CP-asymmetry has an opposite sign with respect to that defined in [65]. We note a wrong sign typo in the last expression in eq. (2.44) in [26] - this can be checked by summing eq. (2.53) of the same article over the flavour indices.
} 
with $x_{j} \equiv M_{j}^{2} / M_{1}^{2}$ and

$$
\xi(x) \equiv \frac{2}{3} x\left[(1+x) \log \left(1+\frac{1}{x}\right)-\frac{2-x}{1-x}\right]
$$

We note that for large $x, \xi(x)=1+\mathcal{O}(1 / x)$, so that, in the hierarchical limit $M_{1} \ll M_{2} \ll M_{3}, \epsilon^{(1)} \propto M_{1}$ since $1 / \sqrt{x_{j}}=M_{1} / M_{j}$ and in this limit $\left(Y^{\dagger} Y\right)_{11} \propto M_{1}$ and $\Im\left[\left(Y^{\dagger} Y\right)_{1 j}^{2}\right] \propto M_{1} M_{j} \cdot{ }^{9}$

Since the Yukawas enter in $\epsilon^{(1)}$ only through the product $Y^{\dagger} Y$, there is no dependence on the PMNS matrix. There is therefore no contribution to $\epsilon^{(1)}$ from the CPV Dirac and Majorana phases in the PMNS matrix.

As the mass scale of leptogenesis is lowered to $M_{1} \sim 10^{12} \mathrm{GeV}$, the single-flavour approximation becomes inaccurate since the SM $\tau$-Yukawa interactions enter in equilibrium during the generation of the lepton asymmetry, i.e. $\Gamma_{\tau} / H z \sim 1$. This is a transition regime, which we will refer to as 1-to-2 flavour transition, where the $\tau$-decoherence effects cannot be neglected. Moreover, as was noticed in [26], when the requisite CP violation in leptogenesis is provided exclusively by the Dirac and/or Majorana CPV phases of the PMNS matrix, the 1-to-2 flavour transition proceeds with an unusual behaviour of the baryon asymmetry $\eta_{B}$, which extends into the region of the unflavoured regime at $M>10^{12} \mathrm{GeV}$. This unusual behaviour will be investigated in detail in our work. Here it suffices to mention that due to $\mathrm{CP}$ violating quantum decoherence effects caused by the SM $\tau$-Yukawa interactions, in which CP violation is provided by the low-energy leptonic CPV phases, the generation of BAU in the single-flavour approximation as described by eqs. (2.24) and (2.25) fails and that the observed BAU can still be generated at $M_{1}>10^{12} \mathrm{GeV}$ even if $\epsilon^{(1)}=0$ and the $\tau$-Yukawa interactions are not in full thermal equilibrium. The basic reason is that the flavour asymmetries evolve differently due to washout differences caused by the $\tau$-Yukawa wash-out interactions.

For $10^{9} \ll T / \mathrm{GeV} \ll 10^{12}$, the $\tau$-Yukawa interactions are in thermal equilibrium while that of $\mu$ are not, namely $\Gamma_{\tau} / H \gg 1$ while $\Gamma_{\mu} / H \ll 1$. Correspondingly, the $\tau$-(anti)lepton state becomes distinguishable from the other flavour states and the coherence in $\left|\psi_{1}\right\rangle\left(\left|\overline{\psi_{1}}\right\rangle\right)$ gets eventually destroyed. As a consequence, the CPV asymmetry in $L_{\tau}$ evolves differently with respect to the asymmetry in the sum of $L_{e}$ and $L_{\mu}$ charges, $L_{\tau^{\perp}} \equiv L_{e+\mu} \equiv L_{e}+L_{\mu}$. This corresponds to the two-flavour regime of leptogenesis or two-flavoured leptogenesis.

For $10^{9} \ll M_{1} / \mathrm{GeV} \ll 10^{12}$, the $\tau$-Yukawa ( $\mu$-Yukawa) interactions enter in thermal equilibrium at $z \ll 1(z \gg 1)$ and leptogenesis can be studied within the two-flavour approximation under which only the $\mu$-decoherence effects are neglected. If in addition the $\tau$-Yukawa interactions are assumed to be infinitely (="sufficiently") fast during the whole period of leptogenesis, the two-flavoured Boltzmann equations (1BE2F) can be used to describe the time-evolution of the CPV asymmetries in the $L_{\tau}$ and $L_{\tau^{\perp}}$ charges and of

\footnotetext{
${ }^{9}$ To be more precise, in $\epsilon^{(1)}$ also factors of the form $f^{-1}\left(M_{2,3}\right) / M_{2,3}$, given in eq. (2.10), appear inside the summation. However, the mass dependence of these factors is logarithmic and, in the mass range $10^{9} \lesssim M / \mathrm{GeV} \lesssim 10^{14}$ of interest to us, $f^{-1}(M) / M$ changes only by a factor of 1.1 taking values in the interval $1.1-1.3$.
} 
BAU. The set of 1BE2F equations in the two-flavour approximation reads:

$$
\begin{aligned}
\frac{d N_{N_{1}}}{d z} & =-D_{1}\left(N_{N_{1}}-N_{N_{1}}^{e q}\right), \\
\frac{d N_{\tau \tau}}{d z} & =\epsilon_{\tau \tau}^{(1)} D_{1}\left(N_{N_{1}}-N_{N_{1}}^{e q}\right)-W_{1} p_{1 \tau} N_{\tau \tau}, \\
\frac{d N_{\tau^{\perp} \tau^{\perp}}}{d z} & =\epsilon_{\tau^{\perp} \tau^{\perp}}^{(1)} D_{1}\left(N_{N_{1}}-N_{N_{1}}^{e q}\right)-W_{1} p_{1 \tau^{\perp}} N_{\tau^{\perp} \tau^{\perp}},
\end{aligned}
$$

where $p_{1 \tau}=\left|C_{1 \tau}\right|^{2}$ and $p_{1 \tau^{\perp}}=\left|C_{1 e}\right|^{2}+\left|C_{1 \mu}\right|^{2}=1-p_{1 \tau}$, while $N_{\tau \tau}$ and $N_{\tau^{\perp} \tau^{\perp}}$ are respectively the values of the asymmetries in the charges $\frac{1}{3} B-L_{\tau}$ and $\frac{2}{3} B-L_{\tau^{\perp}}$ in a comoving volume, so that $N_{B-L}=N_{\tau \tau}+N_{\tau^{\perp} \tau^{\perp}}$. The expressions for the relevant CPV lepton asymmetries $\epsilon_{\tau \tau}^{(1)}$ and $\epsilon_{\tau^{\perp} \tau^{\perp}}^{(1)}=\epsilon_{e e}^{(1)}+\epsilon_{\mu \mu}^{(1)}$ will be given below.

As the mass scale is lowered to $M_{1} \sim 10^{9} \mathrm{GeV}$, leptogenesis approaches the 2-to-3 flavour transition, where the $\mu$-decoherence effects cannot be neglected since the $\mu$-Yukawa interactions enter in equilibrium, $\Gamma_{\mu} / H z \sim 1$. Therefore, the two-flavour approximation ceases to be accurate. Actually, as we are going to show in the present study, there are choices of the parameters for which the 1BE2F equations are never accurate and cannot be used for the description of leptogenesis in the whole range $10^{9} \lesssim M_{1} / \mathrm{GeV} \lesssim 10^{12}$. In addition, in certain regions of the parameter space, the scale below which the 1BE2F set of equations starts to be valid can be significantly lower than $\sim 10^{12} \mathrm{GeV}$.

At $T \ll 10^{9} \mathrm{GeV}$, also the $\mu$-Yukawa interactions are in thermal equilibrium, i.e. $\Gamma_{\mu} / H \gg 1$. This is the three-flavour regime: all the flavours are distinguishable, the coherent superposition in $\left|\psi_{1}\right\rangle\left(\left|\overline{\psi_{1}}\right\rangle\right)$ is fully destroyed and the CPV lepton asymmetries in each of the charges $L_{\alpha}(\alpha=e, \mu, \tau)$ evolve separately. At $M_{1} \ll 10^{9} \mathrm{GeV}$, both the $\mu$ - and $\tau$-Yukawa interactions enter in equilibrium at $z \ll 1$ corresponding to the three-flavoured leptogenesis scenario. If the $\mu$ - and $\tau$-Yukawa interactions are assumed to be infinitely (三"sufficiently") fast, then leptogenesis can be described by the three-flavoured Boltzmann equations (1BE3F), namely:

$$
\begin{aligned}
\frac{d N_{N_{1}}}{d z} & =-D_{1}\left(N_{N_{1}}-N_{N_{1}}^{e q}\right), \\
\frac{d N_{e e}}{d z} & =\epsilon_{e e}^{(1)} D_{1}\left(N_{N_{1}}-N_{N_{1}}^{e q}\right)-W_{1} p_{1 e} N_{e e}, \\
\frac{d N_{\mu \mu}}{d z} & =\epsilon_{\mu \mu}^{(1)} D_{1}\left(N_{N_{1}}-N_{N_{1}}^{e q}\right)-W_{1} p_{1 \mu} N_{\mu \mu}, \\
\frac{d N_{\tau \tau}}{d z} & =\epsilon_{\tau \tau}^{(1)} D_{1}\left(N_{N_{1}}-N_{N_{1}}^{e q}\right)-W_{1} p_{1 \tau} N_{\tau \tau},
\end{aligned}
$$

where $p_{1 \alpha}=\left|C_{1 \alpha}\right|^{2}$, while $N_{\alpha \alpha}$ is the value of the asymmetry in the charge $\frac{1}{3} B-L_{\alpha}$ in a comoving volume, so that $N_{B-L}=\sum_{\alpha} N_{\alpha \alpha}$, with $\alpha=e, \mu, \tau$. 
The CPV lepton asymmetries $\epsilon_{\alpha \alpha}^{(1)}$ in the both set of equations $1 \mathrm{BE} 2 \mathrm{~F}$ and $1 \mathrm{BE} 3 \mathrm{~F}$ are given by $[65-67]:^{10}$

$$
\epsilon_{\alpha \alpha}^{(1)}=\frac{3}{16 \pi\left(Y^{\dagger} Y\right)_{11}} \sum_{j \neq 1}\left\{\Im\left[Y_{\alpha 1}^{*} Y_{\alpha j}\left(Y^{\dagger} Y\right)_{1 j}\right] f_{1}\left(x_{j}\right)+\Im\left[Y_{\alpha 1}^{*} Y_{\alpha j}\left(Y^{\dagger} Y\right)_{j 1}\right] f_{2}\left(x_{j}\right)\right\}
$$

where

$$
f_{1}(x) \equiv \frac{\xi(x)}{\sqrt{x}}, \quad f_{2}(x) \equiv \frac{2}{3(x-1)} .
$$

In eq. (2.39), $\alpha=\tau^{\perp}, \tau$ and $\alpha=e, \mu, \tau$ for the 1BE2F and 1BE3F equations (2.33) - (2.34) and $(2.36)-(2.38)$, respectively, and $\epsilon_{\tau^{\perp} \tau^{\perp}}^{(1)}=\epsilon_{e e}^{(1)}+\epsilon_{\mu \mu}^{(1)}$. We have: $\sum_{\alpha} \epsilon_{\alpha \alpha}^{(1)}=\epsilon^{(1)}$, with $\epsilon^{(1)}$ as given in eq. (2.30).

To obtain a better description of the physics of leptogenesis, the decoherence effects should always be included in the calculations. As already shown in, e.g., [29-31], the density matrix equations (DMEs) provide an accurate tool to study thermal leptogenesis accounting for quantum decoherence processes, especially when these are neither infinitely fast nor their effects negligible. The DMEs describe the time-evolution of the entries of the density matrix, which, in the three-flavour basis, is given by

$$
N=\sum_{\alpha, \beta} N_{\alpha \beta}\left|\psi_{\alpha}\right\rangle\left\langle\psi_{\beta}\right|
$$

with $\alpha, \beta=e, \mu, \tau$. The diagonal entries $N_{\alpha \alpha}$ are the already defined number densities for the $\frac{1}{3} B-L_{\alpha}$ asymmetry, so that $N_{B-L}=\operatorname{Tr}(N)=\sum_{\alpha} N_{\alpha \alpha}$. The off-diagonal elements $N_{\alpha \beta}$ describe the degree of coherence between the flavour states. The DMEs in the three-flavour basis explicitly read [29-31]:

$$
\begin{aligned}
\frac{d N_{N_{1}}}{d z}= & -D_{1}\left(N_{N_{1}}-N_{N_{1}}^{\mathrm{eq}}\right) \\
\frac{d N_{\alpha \beta}}{d z}= & \epsilon_{\alpha \beta}^{(1)} D_{1}\left(N_{N_{1}}-N_{N_{1}}^{\mathrm{eq}}\right)-\frac{1}{2} W_{1}\left\{P^{0(1)}, N\right\}_{\alpha \beta} \\
& -\frac{\Gamma_{\tau}}{H z}\left[I_{\tau},\left[I_{\tau}, N\right]\right]_{\alpha \beta}-\frac{\Gamma_{\mu}}{H z}\left[I_{\mu},\left[I_{\mu}, N\right]\right]_{\alpha \beta},
\end{aligned}
$$

where $I_{\tau}$ and $I_{\mu}$ are $3 \times 3$ matrices such that $\left(I_{\tau}\right)_{\alpha \beta}=\delta_{\alpha \tau} \delta_{\beta \tau}$ and $\left(I_{\mu}\right)_{\alpha \beta}=\delta_{\alpha \mu} \delta_{\beta \mu}$, and

$$
P_{\alpha \beta}^{0(1)} \equiv C_{1 \alpha} C_{1 \beta}^{*},
$$

are projection matrices which generalise the notion of the projection probability. They appear in the anti-commutator structure, which explicitly reads:

$$
\left\{P^{0(1)}, N\right\}_{\alpha \beta}=\sum_{\gamma=e, \mu, \tau}\left(C_{1 \alpha} C_{1 \gamma}^{*} N_{\gamma \beta}+C_{1 \gamma} C_{1 \beta}^{*} N_{\alpha \gamma}\right)
$$

The double-commutator structures in eq. (2.43) give rise to an exponentially damping term proportional to $\Gamma_{\tau, \mu} / \mathrm{Hz}$ for the equations describing the off-diagonal elements of $N$. If

\footnotetext{
${ }^{10}$ The need for a double flavour index will later be clarified as in the quantum treatment also the offdiagonal terms are relevant.
} 
these terms are infinitely large, i.e., $\Gamma_{\tau, \mu} \rightarrow+\infty$, the density matrix is driven towards a diagonal form and the DMEs reduce to the three-flavoured set of Boltzmann equations 1BE3F. The CPV-asymmetry parameters are [12, 29, 31, 65-68]:

$$
\begin{aligned}
\epsilon_{\alpha \beta}^{(1)}= & \frac{3}{32 \pi\left(Y^{\dagger} Y\right)_{11}} \sum_{j \neq 1}\left\{i\left[Y_{\alpha 1} Y_{\beta j}^{*}\left(Y^{\dagger} Y\right)_{j 1}-Y_{\beta 1}^{*} Y_{\alpha j}\left(Y^{\dagger} Y\right)_{1 j}\right] f_{1}\left(x_{j}\right)\right. \\
& \left.+i\left[Y_{\alpha 1} Y_{\beta j}^{*}\left(Y^{\dagger} Y\right)_{1 j}-Y_{\beta 1}^{*} Y_{\alpha j}\left(Y^{\dagger} Y\right)_{j 1}\right] f_{2}\left(x_{j}\right)\right\} .
\end{aligned}
$$

Setting $\alpha=\beta$ in the above expression reproduces the asymmetry $\epsilon_{\alpha \alpha}^{(1)}$ defined in eq. (2.39), while the trace coincides with the expression for $\epsilon^{(1)}$ given in eq. (2.30).

In the numerical analyses that follow, we will use the ULYSSES Python package [69] to solve the sets of equations that we have introduced in the present section. The code computes, in particular, $N_{B-L}=N_{e e}+N_{\mu \mu}+N_{\tau \tau}$, which is then converted into the baryon asymmetry of the Universe $\eta_{B}$ expressed in terms of the baryon-to-photon ratio using the following relation:

$$
\eta_{B}=\frac{28}{79} \frac{1}{27} N_{B-L}
$$

where $28 / 79$ is the SM sphaleron conversion coefficient and the $1 / 27$ factor comes from the dilution of the baryon asymmetry due to the change of the photon density between leptogenesis and recombination [64].

\section{The baryon asymmetry sign change}

We first consider two-flavoured leptogenesis in the case of three heavy Majorana neutrinos $N_{1,2,3}$ with hierarchical masses, $M_{1} \ll M_{2} \ll M_{3}, M_{1} \gtrsim 10^{9} \mathrm{GeV}$. In this case generically only the CPV decays of the lighter Majorana neutrino $N_{1}$ contribute to the generation of CPV lepton asymmetry which is converted into a baryon asymmetry by the sphaleron effects. The density matrix equations (DMEs) describing the evolution of the number of $N_{1}$ in a comoving volume, $N_{N_{1}}$, and of the CPV asymmetries in the lepton charges $L_{\tau}$ and $L_{\tau^{\perp}}=L_{e+\mu}=L_{e}+L_{\mu}$ in the two-flavoured leptogenesis have the following form in the case of interest:

$$
\begin{aligned}
\frac{d N_{N_{1}}}{d z} & =-D_{1}\left(N_{N_{1}}-N_{N_{1}}^{e q}\right), \\
\frac{d N_{\tau \tau}}{d z} & =\epsilon_{\tau \tau}^{(1)} D_{1}\left(N_{N_{1}}-N_{N_{1}}^{e q}\right)-W_{1}\left(p_{1 \tau} N_{\tau \tau}+\Re\left[C_{1 \tau^{\perp}} C_{1 \tau}^{*} N_{\tau \tau^{\perp}}\right]\right), \\
\frac{d N_{\tau^{\perp} \tau^{\perp}}}{d z} & =\epsilon_{\tau^{\perp} \tau^{\perp}}^{(1)} D_{1}\left(N_{N_{1}}-N_{N_{1}}^{e q}\right)-W_{1}\left(p_{1 \tau^{\perp}} N_{\tau^{\perp} \tau^{\perp}}+\Re\left[C_{1 \tau^{\perp}} C_{1 \tau^{\prime}}^{*} N_{\tau \tau^{\perp}}\right]\right), \\
\frac{d N_{\tau \tau^{\perp}}}{d z} & =\epsilon_{\tau \tau^{\perp}}^{(1)} D_{1}\left(N_{N_{1}}-N_{N_{1}}^{e q}\right)-\frac{1}{2} W_{1}\left(N_{\tau \tau^{\perp}}+C_{1 \tau} C_{1 \tau^{\perp}}^{*} N_{B-L}\right)-\frac{\Gamma_{\tau}}{H z} N_{\tau \tau^{\perp}} .
\end{aligned}
$$

The $B-L$ asymmetry is given by $N_{B-L}=N_{\tau \tau}+N_{\tau^{\perp} \tau^{\perp}}$. We find that (see appendix A):

$$
N_{B-L}\left(z_{f}\right)=N_{B-L}^{1 \mathrm{BE} 1 \mathrm{~F}}\left(z_{f}\right)+N_{B-L}^{\mathrm{decoh}}\left(z_{f}\right)
$$


where

$$
\begin{aligned}
N_{B-L}^{1 \mathrm{BE} 1 \mathrm{~F}}(z) & \equiv \int_{z_{0}}^{z} e^{-\int_{z^{\prime}}^{z_{f}} W_{1}\left(z^{\prime \prime}\right) d z^{\prime \prime}} \epsilon^{(1)} D_{1}\left(z^{\prime}\right)\left(N_{N_{1}}\left(z^{\prime}\right)-N_{N_{1}}^{e q}\left(z^{\prime}\right)\right) d z^{\prime} \\
N_{B-L}^{\mathrm{decoh}} & (z) \equiv \int_{z_{0}}^{z} e^{-\int_{z^{\prime}}^{z_{f}} W_{1}\left(z^{\prime \prime}\right) d z^{\prime \prime}} W_{1}\left(z^{\prime}\right) \lambda\left(z^{\prime}\right) d z^{\prime}
\end{aligned}
$$

$z_{0}$ corresponding to the beginning of leptogenesis, which we set to $z_{0}=10^{-3}$ in all our numerical calculations, and

$$
\lambda(z) \equiv 2 \int_{z_{0}}^{z} \Re\left[C_{1 \tau}^{*} C_{1 \tau^{\perp}} N_{\tau \tau^{\perp}}\left(z^{\prime}\right) \frac{\Gamma_{\tau}}{H z^{\prime}}\right] d z^{\prime} .
$$

The term $N_{B-L}^{1 \mathrm{BE} 1 \mathrm{~F}}$ is the solution to the single-flavoured Boltzmann equations (1BE1F) and vanishes if the $\mathrm{CP}$ violation in leptogenesis is due only to the physical Dirac and/or Majorana CPV phases in the PMNS matrix since in that case [18] $\epsilon^{(1)}=\epsilon_{\tau \tau}^{(1)}+\epsilon_{\tau^{\perp} \tau^{\perp}}^{(1)}=$ $\epsilon_{\tau \tau}^{(1)}+\epsilon_{e e}^{(1)}+\epsilon_{\mu \mu}^{(1)}=0$. The term $N_{B-L}^{\text {decoh }}$ incorporates the decoherence effects and one can have $N_{B-L}^{\text {decoh }}>(\gg) N_{B-L}^{1 \mathrm{BE} 1 \mathrm{~F}}$. As was shown in [26], $N_{B-L}^{\text {decoh }}$ can be the only source of CPV lepton asymmetry if the $\mathrm{CP}$ violation in leptogenesis is provided exclusively by the physical CPV phases in the PMNS matrix. In the discussion that follows we focus on this case.

The factor $\Lambda_{\tau} \equiv \Gamma_{\tau} / H z=$ const. $/ M_{1}$ and can be taken out of the integration in (3.8). In the high scale regime $\left(M \gtrsim 10^{12} \mathrm{GeV}\right)$ we can work in the limit of $\Lambda_{\tau} \rightarrow 0$ and neglect all the terms of order $\mathcal{O}\left(\Lambda_{\tau}^{2}\right)$ in the lepton asymmetry. Since in our case $N_{B-L}^{\text {decoh }}$ is the only source of lepton asymmetry, at $M \gtrsim 10^{12} \mathrm{GeV}$ we have $N_{B-L}=N_{B-L}^{\text {decoh }}=\mathcal{O}\left(\Lambda_{\tau}\right)$. Solving eq. (3.4) at zero order in $\Lambda_{\tau},{ }^{11}$ with the integrating factor method we find:

$$
N_{\tau \tau^{\perp}}(z)=\int_{z_{0}}^{z} e^{-\frac{1}{2} \int_{z^{\prime}}^{z} W_{1}\left(z^{\prime \prime}\right) d z^{\prime \prime}} \epsilon_{\tau \tau^{\perp}}^{(1)} D_{1}\left(N_{N_{1}}-N_{N_{1}}^{e q}\right) d z^{\prime}+\mathcal{O}\left(\Lambda_{\tau}\right)
$$

Inserting this result in eq. (3.8) we get:

$$
\lambda(z)=\Lambda_{\tau} \mathcal{I}_{1}\left(\kappa_{1} ; z\right)\left(p_{1 \tau^{\perp}} \epsilon_{\tau \tau}^{(1)}+p_{1 \tau} \epsilon_{\tau^{\perp} \tau^{\perp}}^{(1)}\right)+\mathcal{O}\left(\Lambda_{\tau}^{2}\right)
$$

where

$$
\mathcal{I}_{1}\left(\kappa_{1} ; z\right) \equiv \int_{z_{0}}^{z} d z^{\prime} \int_{z_{0}}^{z^{\prime}} d z^{\prime \prime} e^{-\frac{1}{2} \int_{z_{0}}^{z^{\prime}} W_{1}(\tilde{z}) d \tilde{z}} D_{1}\left(z^{\prime \prime}\right)\left(N_{N_{1}}\left(z^{\prime \prime}\right)-N_{N_{1}}^{e q}\left(z^{\prime \prime}\right)\right)
$$

and we have used the relation $2 \Re\left[C_{1 \tau}^{*} C_{1 \tau^{\perp}} \epsilon_{\tau^{\perp}}\right]=p_{1 \tau^{\perp}} \epsilon_{\tau \tau}^{(1)}+p_{1 \tau} \epsilon_{\tau^{\perp} \tau^{\perp}}^{(1)}$ (see appendix A for a derivation of this relation). To leading order in $\Lambda_{\tau}$ the final asymmetry reads:

$$
N_{B-L}\left(z_{f}\right)=\Lambda_{\tau} \mathcal{I}_{2}\left(\kappa_{1} ; z_{f}\right)\left(p_{1 \tau^{\perp}} \epsilon_{\tau \tau}^{(1)}+p_{1 \tau} \epsilon_{\tau^{\perp} \tau^{\perp}}^{(1)}\right)+\mathcal{O}\left(\Lambda_{\tau}^{2}\right)
$$

where

$$
p_{1 \tau^{\perp}} \epsilon_{\tau \tau}^{(1)}+p_{1 \tau} \epsilon_{\tau^{\perp} \tau^{\perp}}^{(1)}=\left(1-2 p_{1 \tau}\right) \epsilon_{\tau \tau}^{(1)}
$$

and

$$
\mathcal{I}_{2}\left(\kappa_{1} ; z\right) \equiv \int_{z_{0}}^{z} e^{-\int_{z_{0}}^{z} W_{1}(\tilde{z}) d \tilde{z}} W_{1}\left(z^{\prime}\right) \mathcal{I}_{1}\left(\kappa_{1} ; z^{\prime}\right) d z^{\prime}
$$

\footnotetext{
${ }^{11}$ The term $\propto N_{B-L}$ in eq. (3.4) can be neglected since for $\epsilon^{(1)}=0$ it leads to correction $\mathcal{O}\left(\Lambda_{\tau}^{2}\right)$ in the asymmetry.
} 

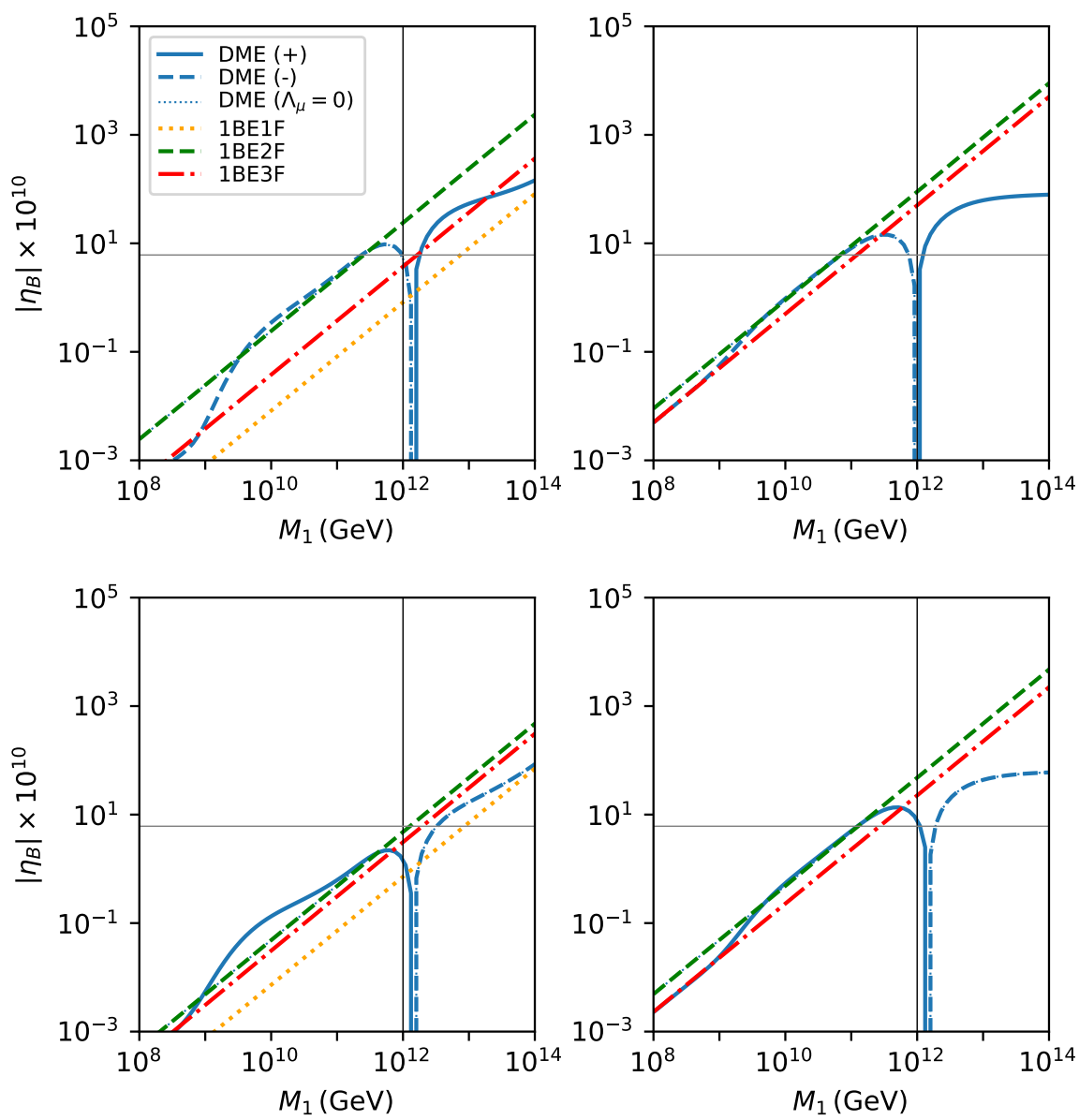

Figure 1. The baryon asymmetry $\left|\eta_{B}\right|$ as a function of the mass scale $M_{1}$ calculated with DME (blue curve), and 1-, 2- and 3-flavoured Boltzmann equations 1BE1F (orange dotted line), 1BE2F (green dashed line) and 1BE3F (red dash-dotted line). The solid (dashed) blue curve corresponds to $\eta_{B}>0\left(\eta_{B}<0\right)$, while the dotted blue curve is obtained for $\Lambda_{\mu}=0$. The lightest neutrino and the heavy Majorana neutrino masses, the Dirac and Majorana CPV phases and Casas-Ibarra parameters are set to: $m_{1}=0.0159 \mathrm{eV}, M_{3}=5 M_{2}=50 M_{1}, \delta=228^{\circ}, \alpha_{21}=200^{\circ}, \alpha_{31}=175^{\circ}$, $x_{1}=-/+10^{\circ}, x_{2}=-/+20^{\circ}, x_{3}=-/+10^{\circ}$ in the top/bottom panels and $y_{1}=y_{2}=0$ and $y_{3}=30^{\circ} / 0$ in the left/right panels, respectively. In the top-right and bottom-right panels the CP violation is due only to the CPV phases in the PMNS matrix $\left(y_{1,2,3}=0\right)$, i.e. $\epsilon^{(1)}=0$, and the corresponding $1 \mathrm{BE} 1 \mathrm{~F}$ solution $\eta_{B}^{1 B E 1 F}=0$. The horizontal (vertical) grey (black) line corresponds to the observed value of $\eta_{B}$ (to $M_{1}=10^{12} \mathrm{GeV}$ ).

Since $\epsilon^{(1)} \propto M_{1}$ and $\Lambda_{\tau} \propto 1 / M_{1}$, the asymmetry given by eq. (3.12) is constant with the mass scale $M_{1}$. Thus, when the CP violation is provided by the CPV phases of the PMNS matrix, at $M_{1}>10^{12} \mathrm{GeV}$ there should exist an interval of values of $M_{1}$ in which the baryon asymmetry $\eta_{B}$ is constant, i.e., does not change with $M_{1}$. Indeed, the numerical solutions of the DMEs show the existence of a plateau at values of $M_{1}>10^{12} \mathrm{GeV}$ [26], as is illustrated in figure 1, right panels. 
We note that if the $\mathrm{CP}$ violation in leptogenesis is due to the Casas-Ibarra matrix and thus $\epsilon^{(1)} \neq 0, N_{B-L}^{1 \mathrm{BE} 1 \mathrm{~F}} \propto M_{1}$ eventually starts to dominate over $N_{B-L}^{\text {decoh }}$ as $M_{1}$ increases, recovering the single-flavour approximation as is clearly seen in figure 1, left panels.

As $M_{1}$ decreases from $M_{1} \sim 10^{12} \mathrm{GeV}, \Lambda_{\tau}$ increases and the solution of DMEs approaches the solution of the two-flavoured Boltzmann equations (1BE2F), which we denote by $N_{B-L}^{1 \mathrm{BE} 2 \mathrm{~F}}$. In the mass range of $10^{9} \lesssim M_{1} / \mathrm{GeV} \lesssim 10^{12}$, the asymmetry is approximately given by $N_{B-L}^{1 \mathrm{BE} 2 \mathrm{~F}}$. However, the transition at $M \sim 10^{12} \mathrm{GeV}$ may take place with a sudden sign change of the baryon asymmetry, as figure 1 shows. The sign change can happen if the solution given in eq. (3.12) has a different sign with respect to the solution $N_{B-L}^{1 \mathrm{BE} 2 \mathrm{~F}} \cdot{ }^{12}$ As figure 1 also indicates, we can have $\eta_{B}>0$ either at $M_{1} \gtrsim 10^{12} \mathrm{GeV}$ or at $M_{1} \lesssim 10^{12} \mathrm{GeV}$. More generally, if we denote by $M_{10}$ the value of $M_{1}$ at which $\eta_{B}=0$, we can have $\eta_{B}>0$ and viable leptogenesis for certain values of $M_{1}$ lying either in the interval $M_{1}>M_{10}$ or in the interval $M_{1}<M_{10}$. Because of the change of the sign of $\eta_{B}$, there is the possibility of finding the predicted $\left|\eta_{B}\right|$ equal to the observed value of the baryon asymmetry but $\eta_{B}$ having the wrong (negative) sign and so no successful leptogenesis (figure 1, bottom-left panel). In view of this, it is of crucial importance to understand the conditions under which $\eta_{B}$ changes sign as well as what determines the value(s) of $M_{1}$ at which $\eta_{B}=0$.

We discuss in the next subsections the circumstances under which the sign change of $\eta_{B}$ can take place in the cases of strong and weak wash-out regimes, for which $\kappa_{1} \gg 1$ and $\kappa_{1} \ll 1$, respectively. We concentrate on the physically interesting possibility of the requisite $\mathrm{CP}$ violation provided only by the Dirac and/or Majorana CPV phases of the PMNS matrix, which leads also to the existence of a "plateau" at $M_{1}>10^{12} \mathrm{GeV}$ where to a good approximation $\eta_{B}$ does not depend on $M_{1}$.

\subsection{Strong wash-out regime}

In the strong wash-out regime the solution to the 1BE2F does not depend on the initial conditions since any initially generated asymmetry is erased by the strong wash-out processes. A sufficiently accurate analytic expression of the solution to the $1 \mathrm{BE} 2 \mathrm{~F}$, valid in the strong wash-out regime, is given by (see appendix B.1):

$$
N_{B-L}^{1 \mathrm{BE} 2 \mathrm{~F}}\left(z_{f}\right) \simeq \frac{2 N_{N_{1}}^{e q}(0)}{\kappa_{1} z_{d}\left(\kappa_{1}\right)} \frac{p_{1 \tau^{\perp}} \epsilon_{\tau \tau}^{(1)}+p_{1 \tau} \epsilon_{\tau^{\perp} \tau^{\perp}}^{(1)}}{p_{1 \tau} p_{1 \tau^{\perp}}}=\frac{2 N_{N_{1}}^{e q}(0)}{\kappa_{1} z_{d}\left(\kappa_{1}\right)} \frac{\left(1-2 p_{1 \tau}\right) \epsilon_{\tau \tau}^{(1)}}{p_{1 \tau}\left(1-p_{1 \tau}\right)} .
$$

Since $p_{1 \tau}\left(1-p_{1 \tau}\right)>0$, a difference in sign between the solution $N_{B-L}^{1 \mathrm{BE} 2 \mathrm{~F}}\left(z_{f}\right)$ given above and the solution of eq. (3.12) occurs when $\mathcal{I}_{2}\left(\kappa_{1} ; z_{f}\right)$ is negative. We show in figure 2 the behaviour of $\mathcal{I}_{2}\left(\kappa_{1} ; z_{f}\right)$ for $z_{f}=1000$ computed numerically with the ULYSSES Python package [69] from eq. (3.13) ${ }^{13}$ in the cases of vanishing initial abundance (VIA) of $N_{1}$, $N_{N_{1}}\left(z_{0}\right)=0$, and thermal initial abundance (TIA) of $N_{1}, N_{N_{1}}\left(z_{0}\right)=N_{N_{1}}^{e q}\left(z_{0}\right)$. As follows

\footnotetext{
${ }^{12} \mathrm{~A}$ sign change of $\eta_{B}$ can occur also at $M \sim 10^{9} \mathrm{GeV}$, where the transition between the two-flavour and three-flavour regimes takes place. If $\epsilon^{(1)} \neq 0$, another sign change can occur at a mass scale $M_{1} \gtrsim 10^{12} \mathrm{GeV}$ when $N_{B-L}^{1 \mathrm{BE} 1 \mathrm{~F}}$ starts dominating over $N_{B-L}^{\text {decoh }}$. However, investigating the conditions under which these sign changes of $\eta_{B}$ take place is beyond the scope of the present study.

${ }^{13}$ We recall that $N_{B-L}(z)$, and therefore also $\mathcal{I}_{2}\left(\kappa_{1} ; z\right)$, is frozen and kept constant after the wash-out processes become ineffective at $z_{d}\left(\kappa_{1}\right) \ll z_{f}$.
} 


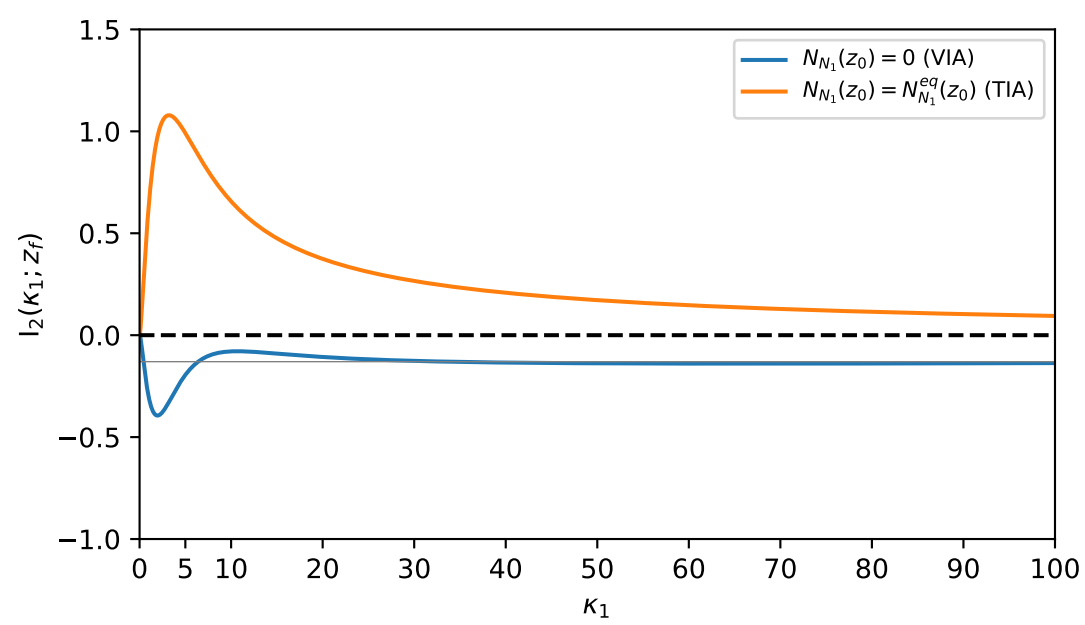

Figure 2. The dependence of the function $\mathcal{I}_{2}\left(\kappa_{1} ; z_{f}\right)$ (defined in eq. (3.13)) on $\kappa_{1}$, computed numerically at $z_{f}=1000$ in the cases of VIA (blue) and TIA (orange) initial abundances of $N_{1}$. Note that in the strong wash-out regime in the VIA case for $k \gtrsim 10$ we have to a good approximation $\mathcal{I}_{2} \approx-0.13$ - the value marked by the horizontal grey line. See text for further details.

from the behaviour of $\mathcal{I}_{2}\left(\kappa_{1} ; z_{f}\right)$ shown in figure 2 , a sign change of $\eta_{B}$ at $M_{1} \sim 10^{12} \mathrm{GeV}$ in the strong wash-out regime always happens for VIA, but never for TIA.

\subsection{Weak wash-out regime}

In the weak wash-out regime we need to consider separately the cases of the two different initial conditions - VIA $\left(N_{N_{1}}\left(z_{0}\right)=0\right)$ and TIA $\left(N_{N_{1}}\left(z_{0}\right)=N_{N_{1}}^{e q}\left(z_{0}\right)\right)$.

\subsubsection{Vanishing initial abundance}

In the VIA case the asymmetry of interest in the two-flavoured leptogenesis reads (see appendix B.2.1):

$$
\begin{aligned}
N_{B-L}^{1 \mathrm{BE} 2 \mathrm{~F}}\left(z_{f}\right) & =\frac{81 \pi^{2}}{1024 N_{\ell}^{e q}} \kappa_{1}^{2}\left(\epsilon_{\tau \tau}^{(1)} p_{1 \tau}+\epsilon_{\tau^{\perp} \tau^{\perp}}^{(1)} p_{1 \tau^{\perp}}\right) \\
& =-\frac{81 \pi^{2}}{1024 N_{\ell}^{e q}} \kappa_{1}^{2} \epsilon_{\tau \tau}^{(1)}\left(p_{1 \tau^{\perp}}-p_{1 \tau}\right) .
\end{aligned}
$$

where to get the last equation we have used the fact that $\epsilon^{(1)}=\epsilon_{\tau \tau}^{(1)}+\epsilon_{\tau^{\perp} \tau^{\perp}}^{(1)}=0$. Using this condition also in eq. (3.12) we find:

$$
N_{B-L}\left(z_{f}\right)=\Lambda_{\tau} \mathcal{I}_{2}\left(\kappa_{1} ; z_{f}\right) \epsilon_{\tau \tau}^{(1)}\left(p_{1 \tau^{\perp}}-p_{1 \tau}\right)+\mathcal{O}\left(\Lambda_{\tau}^{2}\right)
$$

It is then clear from the comparison of the last two equations that $\mathcal{I}_{2}\left(\kappa_{1} ; z_{f}\right)$ needs to be positive in order for a sign change of $\eta_{B}$ to occur at $M_{1} \sim 10^{12} \mathrm{GeV}$. Since, as shown in figure $2, \mathcal{I}_{2}\left(\kappa_{1} ; z_{f}\right)$ is always negative if $N_{N_{1}}\left(z_{0}\right)=0$, the transition at $M \sim 10^{12} \mathrm{GeV}$ in the weak wash-out regime in the VIA case always takes place without a sign change. 


\subsubsection{Thermal initial abundance}

In the case of TIA, for which $N_{N_{1}}\left(z_{0}\right)=N_{N_{1}}^{e q}\left(z_{0}\right)$, and CP violation due only to the CPV phases in the PMNS matrix, the asymmetry of interest in the two-flavoured leptogenesis is described by the following analytic expression (see appendix B.2.2):

$$
N_{B-L}^{1 \mathrm{BE} 2 \mathrm{~F}}\left(z_{f}\right)=\epsilon_{\tau \tau}^{(1)}\left(p_{1 \tau^{\perp}}-p_{1 \tau}\right) \mathcal{A}\left(\kappa_{1} ; z_{f}\right)
$$

where

$$
\mathcal{A}\left(\kappa_{1} ; z\right) \equiv \int_{z_{D}}^{z} d z^{\prime} D_{1}\left(z^{\prime}\right) N_{N_{1}}\left(z^{\prime}\right) \int_{z^{\prime}}^{z} d z^{\prime \prime} W_{1}\left(z^{\prime \prime}\right) d z^{\prime}>0 .
$$

The comparison of (3.17) with eq. (3.16) tells us that no sign change of $\eta_{B}$ should occur in the 1-to-2 flavour transition also in this case, given the fact that $\mathcal{I}_{2}\left(\kappa_{1} ; z_{f}\right)$ is always positive for TIA.

\subsection{Transitions between different flavour regimes: detailed analysis}

The discussion and the results obtained in the preceding subsections led to the important conclusion that we should expect a sign change of the baryon asymmetry at the transition between the single- and two-flavoured leptogenesis in the case of VIA and strong washout regime of baryon asymmetry generation. However, certain important points could not be addressed within the approach used in the discussion leading to this conclusion. For example, the intermediate cases in which the asymmetries in different flavours $N_{\tau \tau}$ and $N_{\tau^{\perp} \tau^{\perp}}$ are generated in different wash-out regimes — strong and weak - could not and have not been considered. The analysis performed by us also does not allow to determine the mass scale $M_{10}$ at which $\eta_{B}=0$ and the transition between the two different flavour regimes considered takes place. Clearly, having different wash-out regimes for the different flavour asymmetries and having a value of $M_{10}$ which differs significantly from $\sim 10^{12} \mathrm{GeV}$, might be possible, in principle, for choices of the parameters, namely the R-matrix angles $x_{1}+i y_{1}, x_{2}+i y_{2}, x_{3}+i y_{3}$, the PMNS phases $\delta, \alpha_{21}, \alpha_{31}$ and the mass of the lightest neutrino $m_{1}$, which differ from the choices considered by us. To address, in particular, the aforementioned points, we use an alternative approach to the problem of interest.

We start from the following equation for $N_{B-L}$ in the case of $\epsilon^{(1)}=0$ (a detailed derivation of this equation is provided in appendix $\mathrm{A}$ ), which is valid as long as $M_{1} \gtrsim$ $10^{9} \mathrm{GeV}$ where $\Lambda_{\mu}$ can be safely neglected:

$$
\frac{d N_{B-L}}{d z}=-W_{1}(z)\left(N_{B-L}(z)-\lambda(z)\right)
$$

The functions $W_{1}(z)$ and $\lambda(z)$ are given in eqs. (2.29) and (3.8), respectively. Within this apparently simple equation for $N_{B-L}$, we have encoded all the decoherence effects on the system in the term $W_{1} \lambda$, which in particular contains both a source and a wash-out term, as will be clarified later on. The term $W_{1} N_{B-L}$ is the usual wash-out term which tends to cancel any initially generated asymmetry. 
Taking into account the expression for $N_{\tau \tau^{\perp}}$ given in eq. (A.10) of appendix A, the function $\lambda(z)$ can be cast in the form:

$$
\begin{aligned}
& \lambda(z)= \\
& \Lambda_{\tau} \epsilon_{\tau \tau}^{(1)}\left(p_{1 \tau^{\perp}}-p_{1 \tau}\right) \int_{z_{0}}^{z} d z^{\prime} \int_{z_{0}}^{z^{\prime}} d z^{\prime \prime} D_{1}\left(z^{\prime \prime}\right)\left(N_{N_{1}}\left(z^{\prime \prime}\right)-N_{N_{1}}^{e q}\left(z^{\prime \prime}\right)\right) e^{-\Lambda_{\tau}\left(z^{\prime}-z^{\prime \prime}\right)} e^{-\frac{1}{2} \int_{z^{\prime \prime}}^{z^{\prime}} d \tilde{z} W_{1}(\tilde{z})} \\
& \quad-\Lambda_{\tau} p_{1 \tau} p_{1 \tau^{\perp}} \int_{z_{0}}^{z} d z^{\prime} \int_{z_{0}}^{z^{\prime}} d z^{\prime \prime} W_{1}\left(z^{\prime \prime}\right) N_{B-L}\left(z^{\prime \prime}\right) e^{-\Lambda_{\tau}\left(z^{\prime}-z^{\prime \prime}\right)} e^{-\frac{1}{2} \int_{z^{\prime \prime}}^{z^{\prime}} d \tilde{z} W_{1}(\tilde{z})} .
\end{aligned}
$$

The first term in this expression for $\lambda(z)$ is the only source of $B-L$ asymmetry, while the second is an integrated wash-out term. In the limit of $\Lambda_{\tau} \rightarrow 0$, i.e., for $M \gg 10^{12} \mathrm{GeV}$, the first term scales as $\Lambda_{\tau}$, while the second term scales as $\Lambda_{\tau}^{2}$ and can be neglected. We note also that the integrated wash-out term can be suppressed by a small value of $p_{1 \tau} p_{1 \tau^{\perp}}$ as well. Given that the source term is proportional to $\Lambda_{\tau} \epsilon_{\tau \tau}^{(1)}\left(p_{1 \tau^{\perp}}-p_{1 \tau}\right)$, also the $B-L$ asymmetry will be proportional to it at any $z$ :

$$
N_{B-L}(z) \equiv \Lambda_{\tau} \epsilon_{\tau \tau}^{(1)}\left(p_{1 \tau^{\perp}}-p_{1 \tau}\right) \tilde{N}_{B-L}(z)
$$

where $\tilde{N}_{B-L}$ can only depend, apart from $z$, on $\kappa_{1}, \Lambda_{\tau}$ and $p_{1 \tau}$ (through the product $\left.p_{1 \tau} p_{1 \tau^{\perp}}\right)$. This means that the function $\lambda(z)$ can be written as:

$$
\lambda(z)=\Lambda_{\tau} \epsilon_{\tau \tau}^{(1)}\left(p_{1 \tau^{\perp}}-p_{1 \tau}\right) \mathcal{S}\left(p_{1 \tau}, \kappa_{1}, \Lambda_{\tau} ; z\right),
$$

with

$$
\begin{aligned}
& \mathcal{S}\left(p_{1 \tau}, \kappa_{1}, \Lambda_{\tau} ; z\right) \equiv \\
& \quad \int_{z_{0}}^{z} d z^{\prime} \int_{z_{0}}^{z^{\prime}} d z^{\prime \prime} D_{1}\left(z^{\prime \prime}\right)\left(N_{N_{1}}\left(z^{\prime \prime}\right)-N_{N_{1}}^{e q}\left(z^{\prime \prime}\right)\right) e^{-\Lambda_{\tau}\left(z^{\prime}-z^{\prime \prime}\right)} e^{-\frac{1}{2} \int_{z^{\prime \prime}}^{z^{\prime}}} d \tilde{z} W_{1}(\tilde{z}) \\
& \quad-\Lambda_{\tau} p_{1 \tau}\left(1-p_{1 \tau}\right) \int_{z_{0}}^{z} d z^{\prime} \int_{z_{0}}^{z^{\prime}} d z^{\prime \prime} W_{1}\left(z^{\prime \prime}\right) \tilde{N}_{B-L}\left(z^{\prime \prime}\right) e^{-\Lambda_{\tau}\left(z^{\prime}-z^{\prime \prime}\right)} e^{-\frac{1}{2} \int_{z^{\prime \prime}}^{z^{\prime}} d \tilde{z} W_{1}(\tilde{z})} .
\end{aligned}
$$

It follows from eqs. (3.19) - (3.23) that in the VIA case of interest we have:

$$
\tilde{N}_{B-L}\left(z_{f}\right)=\int_{z_{0}}^{z_{f}} W_{1}(z) \mathcal{S}\left(p_{1 \tau}, \kappa_{1}, \Lambda_{\tau} ; z\right) e^{-\int_{z}^{z_{f}} W_{1}\left(z^{\prime}\right) d z^{\prime}} d z
$$

The last equation, combined with eq. (3.21), cannot be used to compute the final asymmetry because inside $\mathcal{S}$ a dependence on $\tilde{N}_{B-L}$ is "hidden". However, it is clear from the last expression and eqs. (3.21) and (3.22) that, given the signs of $\epsilon_{\tau \tau}^{(1)}$ and of $\left(p_{1 \tau^{\perp}}-p_{1 \tau}\right)=$ $\left(1-2 p_{1 \tau}\right)$, the sign of the asymmetry $N_{B-L}$ depends on the sign evolution of $\mathcal{S}$. We therefore analyze the behaviour of the function $\mathcal{S}$ to better understand the sign change at the 1-to-2 flavour transition. We construct the function $\mathcal{S}$ by first solving numerically the full set of DMEs with the ULYSSES Python package [69] and then we compute explicitly $\mathcal{S}$ using the definition of $\lambda(z)$ in eq. (3.8) together with eqs. (3.22) and (A.9). ${ }^{14}$

\footnotetext{
${ }^{14}$ The ULYSSES Python code allows to calculate numerically $N_{B-L}, N_{\tau \tau} \perp$ and $\lambda(z)$, which then can be used to obtain $\mathcal{S}$.
} 
We consider the case of heavy Majorana neutrinos having a vanishing initial abundance (VIA), i.e., $N_{N_{1}}\left(z_{0}\right)=0$. At the beginning of leptogenesis at $z>z_{0}$, but $z$ relatively close to $z_{0}$, both the term involving $N_{N_{1}}\left(z^{\prime \prime}\right)$ and the integrated wash-out term in eq. (3.23) are much smaller than the term involving $N_{N_{1}}^{e q}\left(z^{\prime \prime}\right),{ }^{15}$ so that $\mathcal{S}$ starts its evolution with a negative sign. As $z$ increases, $\mathcal{S}$ receives contributions from both terms in eq. (3.23). At values of $z>z_{e q}$, where $z_{e q}$ corresponds to the time of evolution at which $N_{N_{1}}=N_{N_{1}}^{e q}$, we have, as our numerical analysis shows, $N_{N_{1}}(z)-N_{N_{1}}^{e q}(z)>0$. As $z$ increases, the source term in eq. (3.23) goes through zero and becomes positive. Let us call $\tilde{z}_{\Lambda}$ the value of $z$ at which $\mathcal{S}=0$, so that at $z<\tilde{z}_{\Lambda}\left(z>\tilde{z}_{\Lambda}\right)$ we have $\mathcal{S}<0(\mathcal{S}>0)$.

It should be clear from eq. (3.24) that for $z<\tilde{z}_{\Lambda}, \tilde{N}_{B-L}<0$ and therefore also the second (integrated wash-out) term in eq. (3.23) is positive. However, it is significantly smaller than the absolute value of the negative source term involving $N_{N_{1}}^{e q}$. At $z=\tilde{z}_{\Lambda}$, this negative term is compensated by the sum of the source term involving $N_{N_{1}}^{e q}\left(z^{\prime \prime}\right)$ and the integrated wash-out term. At $z>\tilde{z}_{\Lambda}, \mathcal{S}$ is positive and remains so as $z$ increases.

In the TIA case, as our numerical analysis shows, $\tilde{z}_{\Lambda}$ does not exist since, in particular, $N_{N_{1}}(z)>N_{N_{1}}^{e q}(z)$ for $z>z_{0}$ and correspondingly the function $\mathcal{S}$ has a positive sign for the entire period of leptogenesis. This explains why no sign change can be present in the TIA case, as proven in the previous section. In what follows we focus our discussion on the VIA case only.

If in the VIA case the $B-L$ asymmetry is frozen at $z_{f}<\tilde{z}_{\Lambda}$, then, as we have discussed, $\mathcal{S}<0$ and therefore $\tilde{N}_{B-L}\left(z_{f}\right)<0$ (see eq. (3.24)). Thus, we can have $\tilde{N}_{B-L}\left(z_{f}\right)>0$ only if $z_{f}>\tilde{z}_{\Lambda}$.

To highlight this behaviour we focus for a moment on the strong wash-out regime. Suppose that $\kappa_{1} \gg 1$, so that there exist two moments $z_{\text {in }}$ and $z_{\text {out }}$ for which $W_{1}\left(z_{\text {in }}<\right.$ $\left.z<z_{\text {out }}\right) \gtrsim 1$. Then, for $z_{\text {in }}<z<z_{\text {out }}$ to a good approximation we have $d N_{B-L} / d z \cong 0,{ }^{16}$ (see, e.g., [70]), and following the same steps as in appendix B, from eq. (3.19) we get:

$$
N_{B-L}(z) \simeq \lambda(z)
$$

After $z_{\text {out }}$ the asymmetry gets frozen so that:

$$
N_{B-L}(\infty) \simeq \lambda\left(z_{\text {out }}\right)
$$

Hence, as follows from eq. (3.22), the sign of the final asymmetry reads:

$$
\operatorname{sgn}\left(N_{B-L}(\infty)\right)=\left\{\begin{array}{lll}
-\operatorname{sgn}\left(\epsilon_{\tau \tau}^{(1)}\right) \operatorname{sgn}\left(p_{1 \tau^{\perp}}-p_{1 \tau}\right), & \text { if } z_{\text {out }}<\tilde{z}_{\Lambda} \quad(\mathcal{S}<0) ; . \\
\operatorname{sgn}\left(\epsilon_{\tau \tau}^{(1)}\right) \operatorname{sgn}\left(p_{1 \tau^{\perp}}-p_{1 \tau}\right), & \text { if } z_{\text {out }}>\tilde{z}_{\Lambda} \quad(\mathcal{S}>0) .
\end{array} .\right.
$$

At $z_{\text {out }}=\tilde{z}_{\Lambda}$ we have $\mathcal{S}=0$ and therefore $N_{B-L}(\infty)=0$. Analytic expression for both $z_{i n}$ and $z_{\text {out }}$ are given in [64]:

$$
z_{\text {in }}\left(\kappa_{1}\right) \simeq \frac{2}{\sqrt{\kappa_{1}}}, \quad z_{\text {out }} \simeq 1.25 \log \left(25 \kappa_{1}\right) .
$$

\footnotetext{
${ }^{15}$ The integrated wash-out term is negligible because both $W_{1}\left(z^{\prime \prime}\right)$ and $\tilde{N}_{B-L}\left(z^{\prime \prime}\right)$ are strongly suppressed.

${ }^{16}$ We have checked numerically that $d N_{B-L} / d z \cong 0$ is indeed a sufficiently good approximation within the analysis performed by us.
} 
In the weak wash-out regime the analysis is more complicated as the asymmetry may freeze at $z_{f} \neq z_{\text {out }}$ and we do not have any analytic expression for this case. However, on the basis of the numerical analysis we did, we expect leptogenesis to end at $z_{f}$ smaller than a few tens.

We note that $\tilde{z}_{\Lambda}$ depends only on $p_{1 \tau}, \kappa_{1}$ and $\Lambda_{\tau}$, i.e. $\tilde{z}_{\Lambda}=\tilde{z}_{\Lambda}\left(p_{1 \tau}, \kappa_{1}, \Lambda_{\tau}\right)$. If we neglect the weak dependence on the mass scale $M_{1}$ of $\kappa_{1}$, which comes from the loop contribution to the light neutrino masses [25], the only dependence of $\tilde{z}_{\Lambda}$ on $M_{1}$ is inside $\Lambda_{\tau} \propto 1 / M_{1}$. Therefore we have ${ }^{17} \tilde{z}_{\Lambda} \simeq \tilde{z}_{\Lambda}\left(p_{1 \tau}, \kappa_{1}, M_{1}\right)$. In addition, in the limit of $\Lambda_{\tau} \rightarrow 0$, i.e., at $M_{1} \gg 10^{12} \mathrm{GeV}$, the integrated wash-out term - the second term in eq. (3.23) can be neglected so that the dependence of $\mathcal{S}$, and therefore of $\tilde{z}_{\Lambda}$, on $p_{1 \tau}$ drops off, i.e., $\tilde{z}_{\Lambda} \simeq \tilde{z}_{\Lambda}\left(\kappa_{1}, M_{1}\right)$. In terms of the Casas-Ibarra parametrisation this means that $\tilde{z}_{\Lambda}$ does not depend on the PMNS phases. As the mass scale $M_{1}$ decreases, the integrated wash-out term becomes non-negligible activating a dependence on the PMNS phases through the product $p_{1 \tau} p_{1 \tau^{\perp}}$.

In the general case of three heavy Majorana neutrinos having non-generate but also non-hierarchical masses (e.g., $M_{3}=3 M_{2}, M_{2}=3 M_{1}$ ), the discussion is rather complicated due to the large number of parameters present in the Casas-Ibarra parametrisation. To make the discussion as transparent as possible we consider the case of decoupled $N_{3}$, in which the number of parameters is significantly smaller than in the general case.

\section{The case of decoupled $N_{3}$}

In the case of decoupled heavy Majorana neutrino $N_{3}\left(M_{1} \ll M_{2} \ll M_{3}\right)$, the lightest neutrino, as is well known, is massless at tree and one loop level, i.e., $m_{1} \cong 0\left(m_{3} \cong 0\right)$, and the light neutrino mass spectrum is normal (inverted) hierarchical, denoted as $\mathrm{NH}$ (IH). The set of parameters relevant for our discussion includes: the masses of the two heavy Majorana neutrinos $M_{1}$ and $M_{2}$; the three CPV phases $\delta, \alpha_{21}, \alpha_{31}$ of the PMNS matrix; the real and imaginary parts $x$ and $y$ of the complex angle of the Casas-Ibarra orthogonal $R$-matrix. The $R$-matrix for the $\mathrm{NH}$ and $\mathrm{IH}$ light neutrino mass spectra of interest has the form:

$$
\begin{aligned}
R^{(\mathrm{NH})}=\left(\begin{array}{ccc}
0 & R_{12} & R_{13} \\
0 & R_{22} & R_{23} \\
1 & 0 & 0
\end{array}\right)=\left(\begin{array}{ccc}
0 & \cos \theta & \sin \theta \\
0 & -\sin \theta & \cos \theta \\
1 & 0 & 0
\end{array}\right), \\
R^{(\mathrm{IH})}=\left(\begin{array}{ccc}
R_{11} & R_{12} & 0 \\
R_{21} & R_{22} & 0 \\
0 & 0 & 1
\end{array}\right)=\left(\begin{array}{ccc}
\cos \theta & \sin \theta & 0 \\
-\sin \theta & \cos \theta & 0 \\
0 & 0 & 1
\end{array}\right),
\end{aligned}
$$

with $\theta=x+i y$. Both $R$-matrices in eqs. (4.1) and (4.2) have $\operatorname{det}(R)=1$. In the literature, the factor $\varphi= \pm 1$ is sometimes included in the definition of $R$ to allow for the both cases $\operatorname{det}(R)= \pm 1$. We choose instead to work with matrices in eqs. (4.1) and (4.2) but extend the range of the Majorana phases $\alpha_{21(31)}$ from $[0,2 \pi]$ to $[0,4 \pi]$, which effectively accounts for the case of $\operatorname{det}(R)=-1$ [22], so that the same full set of $R$ and Yukawa matrices is considered.

\footnotetext{
${ }^{17}$ Approximate analytic expression for the value of $M_{10}$ at which $\tilde{z}_{\Lambda}=0$ and the 1-to-2 flavour transition takes place is derived in appendix $\mathrm{C}$.
} 
In what follows we will analyse the case of hierarchical mass spectrum of the two heavy Majorana neutrinos, $M_{1} \ll M_{2}$. In the subsequent numerical analysis we use $M_{2}=10 M_{1}$.

\subsection{CP violation from low-energy CPV phases of the PMNS matrix}

We are interested in the scenario of leptogenesis in which the CP violation is due exclusively to the low-energy CPV phases present in the PMNS matrix. Correspondingly, we should avoid contributions to $\mathrm{CP}$ violation in leptogenesis associated with the $R$-matrix. To satisfy this requirement we can [18] either set i) $y=0$ and $x \neq 0$, which corresponds to a real $R$-matrix; or 2) $x=k \pi, k=0,1,2$, and $y \neq 0$, so that in the case of NH (IH) spectrum the product $R_{12} R_{13}\left(R_{11} R_{12}\right)$ of the $R$-matrix elements (see eqs. (4.1) and (4.2)), which enters into the expression for the CPV asymmetry $\epsilon_{\tau \tau}^{(1)}$, is purely imaginary.

We first report the expressions for $\kappa_{1}, p_{1 \tau}$ and $\epsilon_{\tau \tau}^{(1)}$ in the NH case for $y=0$ (real $\left.R_{12} R_{13}\right)$, relevant for our further analysis:

$$
\begin{aligned}
k_{1} & =\frac{1}{m_{*}} \frac{f^{-1}\left(M_{1}\right)}{M_{1}}\left(m_{2} \cos ^{2} x+m_{3} \sin ^{2} x\right), \\
p_{1 \tau} & =\frac{m_{2}\left|U_{\tau 2}\right|^{2} \cos ^{2} x+m_{3}\left|U_{\tau 3}\right|^{2} \sin ^{2} x+\sqrt{m_{2} m_{3}} \Re\left(U_{\tau 2}^{*} U_{\tau 3}\right) \sin 2 x}{m_{2} \cos ^{2} x+m_{3} \sin ^{2} x}, \\
\epsilon_{\tau \tau}^{(1)} & =\frac{3 M_{1}}{16 \pi v^{2}} \frac{f^{-1}\left(M_{2}\right)}{M_{2}} \frac{\sqrt{m_{2} m_{3}}\left(m_{3}-m_{2}\right) \sin 2 x}{m_{2} \cos ^{2} x+m_{3} \sin ^{2} x} \Im\left(U_{\tau 2}^{*} U_{\tau 3}\right)+\mathcal{O}\left(\frac{M_{1}}{M_{2}}\right),
\end{aligned}
$$

where $m_{*}$ and $f\left(M_{1,2}\right)$ are defined in eqs. (2.28) and (2.10). The corresponding expressions for the IH spectrum can formally be obtained from those given above by changing $m_{2(3)} \rightarrow$ $m_{1(2)}$ and $U_{\tau 2(\tau 3)} \rightarrow U_{\tau 1(\tau 2)}$.

For the $M_{1}$ and $M_{2}$ mass ranges we are going to consider, namely $M_{1}=\left(10^{9}-\right.$ $\left.10^{13}\right) \mathrm{GeV}, M_{2}=10 M_{1}$, the factors $f^{-1}\left(M_{1}\right) / M_{1}$ and $f^{-1}\left(M_{2}\right) / M_{2}$ in the expressions for $k_{1}$ and $\epsilon_{\tau \tau}^{(1)}$ vary slowly in the intervals $1.16-1.27$ and $1.19-1.30$, respectively, increasing from the minimal values as $M_{1}$ and $M_{2}$ increase.

The combinations of the PMNS entries that appear in equations (4.3) - (4.5) are given by:

$$
\begin{aligned}
\left|U_{\tau 1}\right|^{2}= & s_{12}^{2} s_{23}^{2}+c_{12}^{2} c_{23}^{2} s_{13}^{2}-2 s_{12} c_{12} s_{23} c_{23} s_{13} \cos \delta \\
\left|U_{\tau 2}\right|^{2}= & c_{12}^{2} s_{23}^{2}+s_{12}^{2} c_{23}^{2} s_{13}^{2}+2 s_{12} c_{12} s_{23} c_{23} s_{13} \cos \delta \\
\left|U_{\tau 3}\right|^{2}= & c_{23}^{2} c_{13}^{2}, \\
\Im\left(U_{\tau 2}^{*} U_{\tau 3}\right)= & c_{23} c_{13}\left[c_{12} s_{23} \sin \left(\frac{\alpha_{21}-\alpha_{31}}{2}\right)+s_{12} c_{23} s_{13} \sin \left(\frac{\alpha_{21}-\alpha_{31}}{2}+\delta\right)\right], \\
\Re\left(U_{\tau 2}^{*} U_{\tau 3}\right)= & -c_{23} c_{13}\left[c_{12} s_{23} \cos \left(\frac{\alpha_{21}-\alpha_{31}}{2}\right)+s_{12} c_{23} s_{13} \cos \left(\frac{\alpha_{21}-\alpha_{31}}{2}+\delta\right)\right], \\
\Im\left(U_{\tau 1}^{*} U_{\tau 2}\right)= & -s_{12} c_{12}\left(s_{23}^{2}-c_{23}^{2} s_{13}^{2}\right) \sin \left(\frac{\alpha_{21}}{2}\right)+ \\
& -s_{23} c_{23} s_{13}\left[c_{12}^{2} \sin \left(\delta-\frac{\alpha_{21}}{2}\right)+s_{12}^{2} \sin \left(\frac{\alpha_{21}}{2}+\delta\right)\right], \\
\Re\left(U_{\tau 1}^{*} U_{\tau 2}\right)= & -s_{12} c_{12}\left(s_{23}^{2}-c_{23}^{2} s_{13}^{2}\right) \cos \left(\frac{\alpha_{21}}{2}\right) \\
& +s_{23} c_{23} s_{13}\left[c_{12}^{2} \cos \left(\delta-\frac{\alpha_{21}}{2}\right)-s_{12}^{2} \cos \left(\frac{\alpha_{21}}{2}+\delta\right)\right] .
\end{aligned}
$$




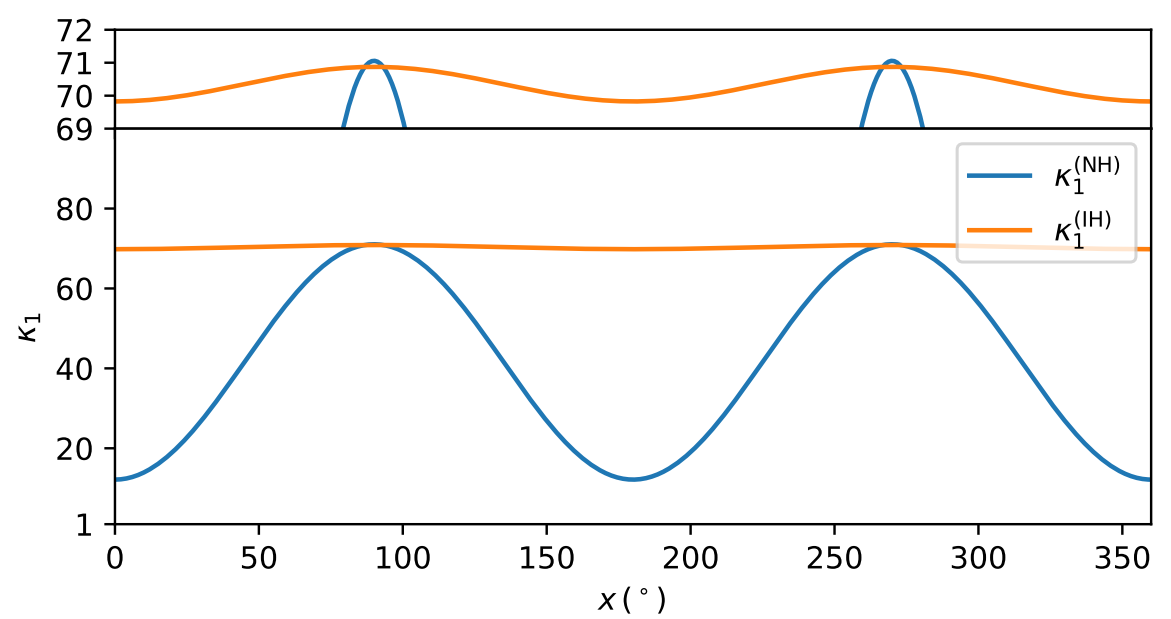

Figure 3. The decay parameter $\kappa_{1}$ versus $x$ for NH (blue) and IH (orange) light neutrino mass spectra with real $R$-matrix, i.e., $y=0$. As the figure shows, $\kappa_{1} \gg 1$, meaning that leptogenesis occurs always in the strong wash-out regime. The figure illustrates also the periodicity of $\pi$ in the $\kappa_{1}$ dependence on $x$. The top panel illustrates the small oscillations of the IH curve.

In the NH (IH) case, only the Majorana CPV phase difference (phase) $\alpha_{21}-\alpha_{31}\left(\alpha_{21}\right)$ is physically relevant. ${ }^{18}$ We note also that the dependence on $\delta$ is always suppressed by $\sin \theta_{13}$. Thus, for the NH (IH) neutrino mass spectrum $\tilde{z}_{\Lambda}$ is predominantly a function of the Majorana phase $\alpha_{23}\left(\alpha_{21}\right)$, of the real part of the $R$-matrix angle $x$ and of the mass scale $M_{1}$, exhibiting also subleading dependence on $\delta$.

For the CP conserving values of the Dirac and Majorana phases, $\delta=0, \pi, \alpha_{21}=k_{21} \pi$ and $\alpha_{31}=k_{31} \pi, k_{21}=0,1,2, \ldots, k_{31}=0,1,2, \ldots$, with $\alpha_{23} \neq \pm 2 n \pi\left(\alpha_{21} \neq 2 n \pi\right), n=$ $0,1,2$, in the $\mathrm{NH}(\mathrm{IH})$ case, and real values of the elements of the $R$-matrix, $x \neq 0, y=0$, the CP-symmetry is nevertheless violated in leptogenesis due to the interplay of the $\mathrm{CP}$ conserving PMNS and real $R$ matrices [18] and $\epsilon_{\tau \tau}^{(1)} \neq 0$, as also follows from eqs. (4.5), (4.9) and (4.11).

We present graphically in figure 3 the dependence of the decay parameter $\kappa_{1}$ on $x$ for $\mathrm{NH}$ and $\mathrm{IH}$ neutrino mass spectra when $y=0$. As figure 3 shows, in both the $\mathrm{NH}$ and $\mathrm{IH}$ cases leptogenesis occurs in the strong wash-out regime, i.e., $\kappa_{1} \gg 1$ for any choice of $x$. We can therefore rely on eq. (3.27) to study the change of sign of $\eta_{B}$ in the 1-to-2 flavour transition when $y=0$ and $x \neq 0$.

For $y=0$ and $x \neq 0$, i.e., for real elements of the $R$-matrix, the final baryon asymmetry $\eta_{B}$ is always suppressed in the IH case with respect to that in the NH case [18]. This is a consequence of the fact that the CPV asymmetry $\epsilon_{\tau \tau}^{(1)}$ in the $\mathrm{NH}$ and $\mathrm{IH}$ cases, $\epsilon^{(\mathrm{NH})}$ and $\epsilon^{(\mathrm{IH})}$, are proportional respectively to $m_{3}-m_{2}$ and $m_{2}-m_{1}$ (see eq. (4.5) and the subsequent discussion), $m_{3,2} \equiv m_{3,2}(\mathrm{NH})$ and $m_{2,1} \equiv m_{2,1}(\mathrm{IH})$ being the corresponding

\footnotetext{
${ }^{18}$ We will call the Majorana phase difference $\alpha_{23}$ simply "Majorana phase" and will denote it as $\alpha_{23}$ in what follows.
} 


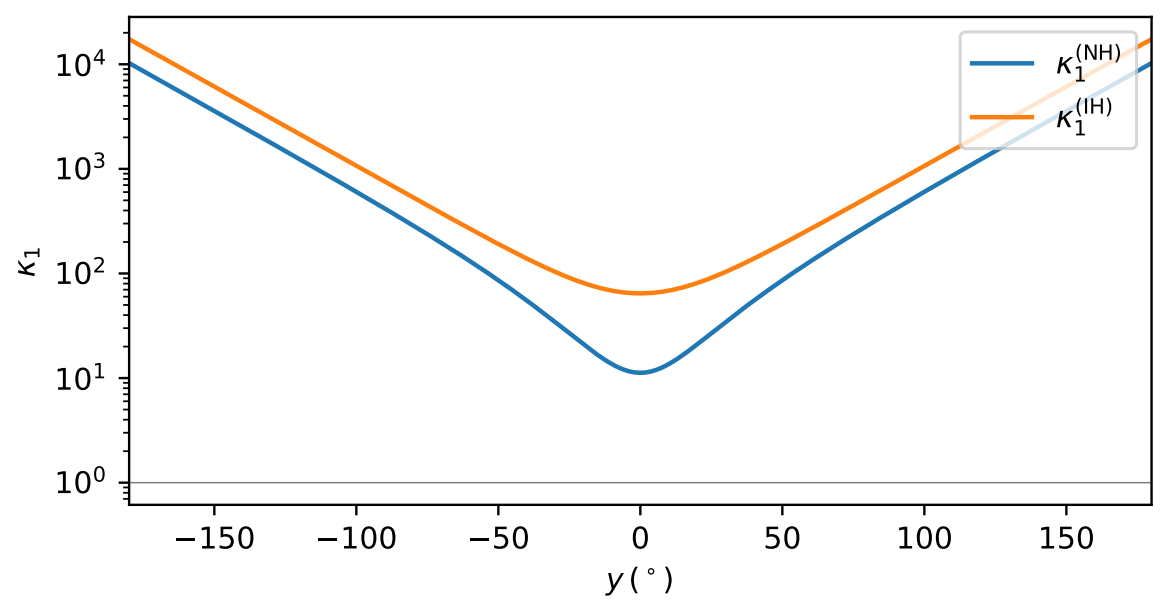

Figure 4. The decay parameter $\kappa_{1}$ as a function of $y$ for $\mathrm{NH}$ (blue) and IH (orange) light neutrino mass spectra in the case of purely imaginary product $R_{12} R_{13}\left(R_{11} R_{12}\right)$, i.e., $x=0, \pi, \ldots$. As the figure shows, $\kappa_{1} \gg 1$, meaning that leptogenesis always takes place in the strong wash-out regime.

light neutrino masses of the two spectra (see section 2.1), so that

$$
\frac{\left|\epsilon^{(\mathrm{IH})}\right|}{\left|\epsilon^{(\mathrm{NH})}\right|} \propto \frac{1}{2}\left(\frac{\Delta m_{21}^{2}}{\Delta m_{\mathrm{atm}}^{2}}\right)^{3 / 4} \approx \frac{1}{30} .
$$

As a consequence, it is impossible to have a successful leptogenesis for IH neutrino mass spectrum with CP violation provided only by the CPV phases in the PMNS matrix and real $R$-matrix for $M_{1} \lesssim 10^{13} \mathrm{GeV}$. The suppression can be avoided in the considered scenario if the product $R_{11} R_{12}$ of the $R$-matrix elements is purely imaginary [18], i.e., if $x=k \pi$, $k=0,1,2$, and $y \neq 0$. Under the conditions $x=k \pi$ and $y \neq 0$, the expressions for $\kappa_{1}, p_{1 \tau}$ and $\epsilon_{\tau \tau}^{(1)}$ in the IH case take the form:

$$
\begin{aligned}
k_{1} & =\frac{1}{m_{*}} \frac{f^{-1}\left(M_{1}\right)}{M_{1}}\left(m_{1} \cosh ^{2} y+m_{2} \sinh ^{2} y\right), \\
p_{1 \tau} & =\frac{m_{1}\left|U_{\tau 1}\right|^{2} \cosh ^{2} y+m_{2}\left|U_{\tau 2}\right|^{2} \sinh ^{2} y-\sqrt{m_{1} m_{2}} \Im\left(U_{\tau 1}^{*} U_{\tau 2}\right) \sinh 2 y}{m_{1} \cosh ^{2} y+m_{2} \sinh ^{2} y}, \\
\epsilon_{\tau \tau}^{(1)} & =-\frac{3 M_{1}}{16 \pi v^{2}} \frac{f^{-1}\left(M_{2}\right)}{M_{2}} \frac{\sqrt{m_{1} m_{2}}\left(m_{2}+m_{1}\right) \sinh 2 y}{m_{1} \cosh ^{2} y+m_{2} \sinh ^{2} y} \Re\left(U_{\tau 1}^{*} U_{\tau 2}\right)+\mathcal{O}\left(\frac{M_{1}}{M_{2}}\right) .
\end{aligned}
$$

The corresponding expressions for the NH spectrum can formally be obtained from those given above by changing $m_{1(2)} \rightarrow m_{2(3)}$ and $U_{\tau 1(\tau 2)} \rightarrow U_{\tau 2(\tau 3)}$.

The suppression of $\eta_{B}$ in the IH case is now avoided due to the presence of the factor $\left(m_{1}+m_{2}\right)$ in the CPV-asymmetry $\epsilon_{\tau \tau}^{(1)}$.

For $x=k \pi, k=0,1,2$, and $y \neq 0$, the strong wash-out condition $\kappa_{1} \gg 1$ is always satisfied, as is shown in figure 4. Thus, also in this case we can rely on eq. (3.27) to discuss the sign change of $\eta_{B}$ at the 1-to-2 flavour transition. 

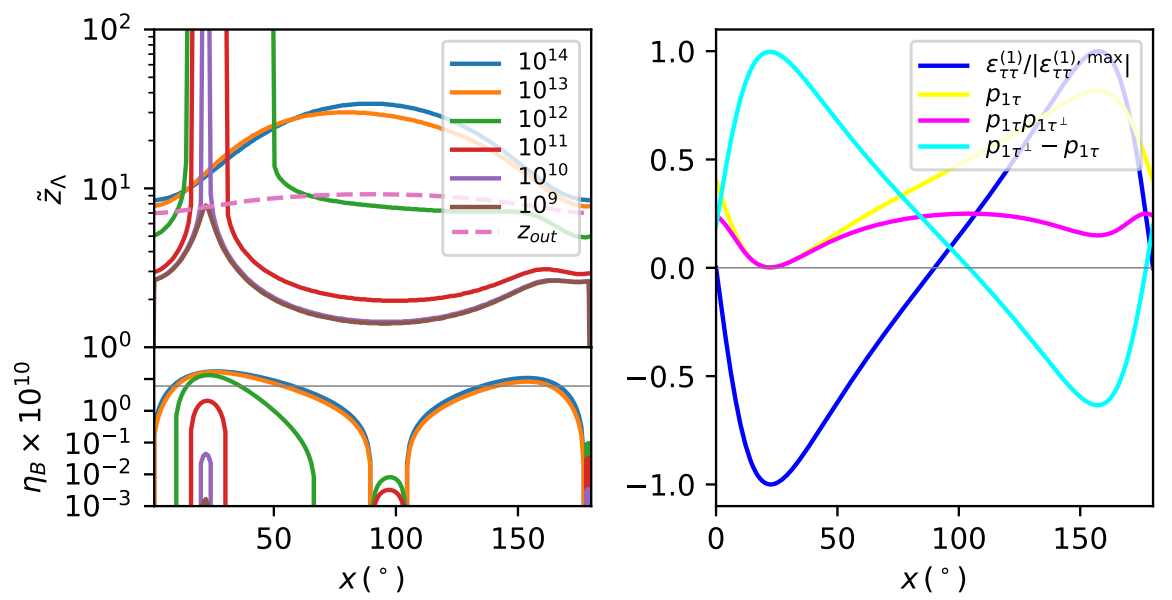

Figure 5. Top-left panel: $\tilde{z}_{\Lambda}$ versus $x$ for different mass scales $M_{1}=10^{14}$ (blue), $10^{13}$ (orange), $10^{12}$ (green), $10^{11}$ (red), $10^{10}$ (purple), $10^{9}$ (brown) GeV. The dashed pink line corresponds to $z_{\text {out }}$, when the generated $\eta_{B}$ gets frozen. Bottom-left panel: the final baryon asymmetry (with the correct positive sign) $\eta_{B}$ versus $x$ for the set of values of $M_{1}$ used to obtain the top panel. The horizontal grey line marks the present BAU at $6.1 \times 10^{-10}$. The right panel shows $\epsilon_{\tau \tau}^{(1)}$ normalised to its maximal value (dark blue), $p_{1 \tau}$ (yellow), $p_{1 \tau} p_{1 \tau^{\perp}}$ (magenta) and $p_{1 \tau^{\perp}}-p_{1 \tau}$ (cyan). The sign of $\epsilon_{\tau \tau}^{(1)}\left(p_{1 \tau^{\perp}}-p_{1 \tau}\right)$ is related to the sign of $\eta_{B}$ via eq. (3.27). The plots are obtained for $M_{2}=10 M_{1}$, $\delta=3 \pi / 2, \alpha_{21}=\alpha_{31}=0$ and NH spectrum. See text for further details.

For the $R$-matrix corresponding to the $\mathrm{NH}(\mathrm{IH})$ spectrum with elements $R_{12}$ and $R_{13}\left(R_{11}\right.$ and $\left.R_{12}\right)$ whose product is purely imaginary, i.e., $x=k \pi$ and $y \neq 0$, the CPsymmetry can also be violated in leptogenesis due to the interplay of the $R$-matrix and the CP conserving PMNS matrix [18]. This possibility is realised for the CP conserving values of the Dirac and Majorana phases, $\delta=0, \pi, \alpha_{21}=k_{21} \pi$ and $\alpha_{31}=k_{31} \pi, k_{21}=0,1,2, \ldots$, $k_{31}=0,1,2, \ldots$, with $\alpha_{23} \neq \pm(2 n+1) \pi\left(\alpha_{21} \neq(2 n+1) \pi\right), n=0,1$, in the NH (IH) case. Under these conditions we have $\epsilon_{\tau \tau}^{(1)} \neq 0$, as also follows from eqs. (4.16), (4.10) and (4.12).

Given that the asymmetry $\eta_{B}$ is approximately constant in the plateau region at $M_{1} \gtrsim 3 \times 10^{12} \mathrm{GeV}$ and decreases with the mass scale (see, e.g., figure 1 and the discussion in section 3 ), the condition ensuring that $\eta_{B}$ is greater than or equal to the present BAU corresponds to the region of the parameter space for which we can have successful leptogenesis at $M_{1} \gtrsim 3 \times 10^{12} \mathrm{GeV}$. Using eqs. (2.18), (2.47) and (3.12), together with the fact that $\mathcal{I}_{2}\left(k_{1} ; z_{f}\right) \approx-0.13$ in the strong wash-out regime in the VIA case (see figure 2 ), we get for the condition of interest:

$$
\eta_{B}=-0.13 \frac{28}{79} \frac{1}{27} \Lambda_{\tau} \epsilon_{\tau \tau}^{(1)}\left(1-2 p_{1 \tau}\right) \gtrsim 6.1 \times 10^{-10} .
$$

\subsection{CP violation due to the Dirac phase}

We consider in this subsection the scenario of leptogenesis with decoupled $N_{3}$ and CP violation due only to the Dirac phase $\delta$. To this end the Majorana phase $\alpha_{23}\left(\alpha_{21}\right)$ is set 
in the $\mathrm{NH}(\mathrm{IH})$ case to the following $\mathrm{CP}$ conserving values: i) $\pm 2 n \pi(2 n \pi), n=0,1,2$, when $x \neq 0$ and $y=0$; ii) $\pm(2 n+1) \pi((2 n+1) \pi), n=0,1$, when $x=k \pi, k=0,1,2$, and $y \neq 0$. With the choices of the values of $\alpha_{23}\left(\alpha_{21}\right)$ made we avoid the situation in which one of the sources of $\mathrm{CP}$ violation in leptogenesis is the interplay of $\mathrm{CP}$ conserving Majorana phases and $R$-matrix elements [18] that could be generated by the first term in the r.h.s. of eq. (4.9) (eq. (4.11)).

\subsubsection{The case of real $R$-matrix $(x \neq 0$ and $y=0)$}

We show in the top-left panel of figure 5 the curves of $\tilde{z}_{\Lambda}$ versus the angle $x$ for different mass scales $M_{1}$ assuming NH mass spectrum. The other parameters are set to: $\delta=3 \pi / 2$, $\alpha_{23}=0$ and $y=0$. The interpretation of the figure in this panel on the basis of eq. (3.27) should be the following. The values of $M_{1}$ and $x$ for which the $\tilde{z}_{\Lambda}$ curve lies above the $z_{\text {out }}$ curve correspond to $\operatorname{sgn}\left(N_{B-L}(\infty)\right)=-\operatorname{sgn}\left(\epsilon_{\tau \tau}^{(1)}\right) \operatorname{sgn}\left(p_{1 \tau^{\perp}}-p_{1 \tau}\right)$. Alternatively, if the $\tilde{z}_{\Lambda}$ curve lies below the $z_{\text {out }}$ one, then $\operatorname{sgn}\left(N_{B-L}(\infty)\right)=\operatorname{sgn}\left(\epsilon_{\tau \tau}^{(1)}\right) \operatorname{sgn}\left(p_{1 \tau^{\perp}}-p_{1 \tau}\right)$. The intersection points correspond to a vanishing $\eta_{B}$ and mark the 1-to-2 flavour transition. In the bottom-left panel the generated baryon asymmetry $\eta_{B}$ (with the correct sign) at different mass scales $M_{1}$ is also depicted. In the right panel we show the behaviour with $x$ of the relevant quantities: $\epsilon_{\tau \tau}^{(1)}$ (normalised to its absolute maximal value), $p_{1 \tau}, p_{1 \tau} p_{1 \tau^{\perp}}$ and $p_{1 \tau^{\perp}}-p_{1 \tau}$.

For the considered choice of the parameters, depending on $x$ we can have different scenarios. At $x \lesssim 10^{\circ}$ and $x \gtrsim 60^{\circ}$, the $10^{13,14} \mathrm{GeV}$ curves lie above the $z_{\text {out }}$ line while the $10^{12,11,10,9} \mathrm{GeV}$ curves lie below (with the $10^{12} \mathrm{GeV}$ curve lying near the $z_{\text {out }}$ line). We can conclude that, for $x \lesssim 10^{\circ}$ and $x \gtrsim 60^{\circ}$, the 1-to- 2 flavour transition occurs at values of $M_{1}$ slightly larger than $10^{12} \mathrm{GeV}$ and with a sign change. According to the bottom-left panel, in the indicated ranges of $x$ we can have successful leptogenesis for values of $x \approx 150^{\circ}$ at $M \gtrsim 10^{13} \mathrm{GeV}$. At $x^{\mathrm{min}} \simeq 22.2^{\circ}$ the magenta curve in the right panel of figure 5 , corresponding to $p_{1 \tau} p_{1 \tau^{\perp}}$, reaches an absolute minimum for the chosen value of $\alpha_{23}=0$. We then note that in the range $10^{\circ} \lesssim x \lesssim 60^{\circ}$, the transition occurs at $M \lesssim 10^{12} \mathrm{GeV}$, and the more the range of $x$ is squeezed around $x^{\mathrm{min}}$, the lower is the mass scale of the transition. At $x^{\text {min }}$ no sign change occurs at the transition, given that all the $\tilde{z}_{\Lambda}$ curves obtained for $M_{1}>10^{9} \mathrm{GeV}$ lie above the $z_{\text {out }}$ line. As is shown in the bottom-left panel of figure 5 , at $x^{\text {min }}$ the final baryon asymmetry $\eta_{B}$ is positive at any mass scale and reaches the observed value at $10^{11} \lesssim M_{1} / \mathrm{GeV} \lesssim 10^{12}$.

In figure 6 we plot $\eta_{B}$ as a function of $M_{1}$, calculated using the density matrix equations (DMEs), two-flavoured (1BEF2) and three-flavoured (1BEF3) Boltzmann equations, for different values of $x$, namely $x=150^{\circ}$ (top-left panel), $40^{\circ}$ (top-right panel), 30 (bottomleft panel) and $22.2^{\circ}$ (bottom-right panel). The PMNS phases and $M_{2}$ are the same as in figure 5. The solid (dashed) blue curve corresponds to $\eta_{B}>0\left(\eta_{B}<0\right)$ and is obtained including the contributions from the $\mu$-Yukawa interactions, thus allowing to account for the 2-to-3 flavour transition when using the DMEs. The sign change of $\eta_{B}$ at the 1-to-2 (2to-3) flavour transition is present in both the top and the bottom-left (in the top-right and bottom-left) panels. The mass scale $M_{1}$ of the 1-to-2 (2-to-3) flavour transition decreases with $x$ as it approaches the value $x^{\mathrm{min}}=22.2^{\circ}$ (stays essentially constant at $\left.M_{1} \simeq 10^{9} \mathrm{GeV}\right)$. 

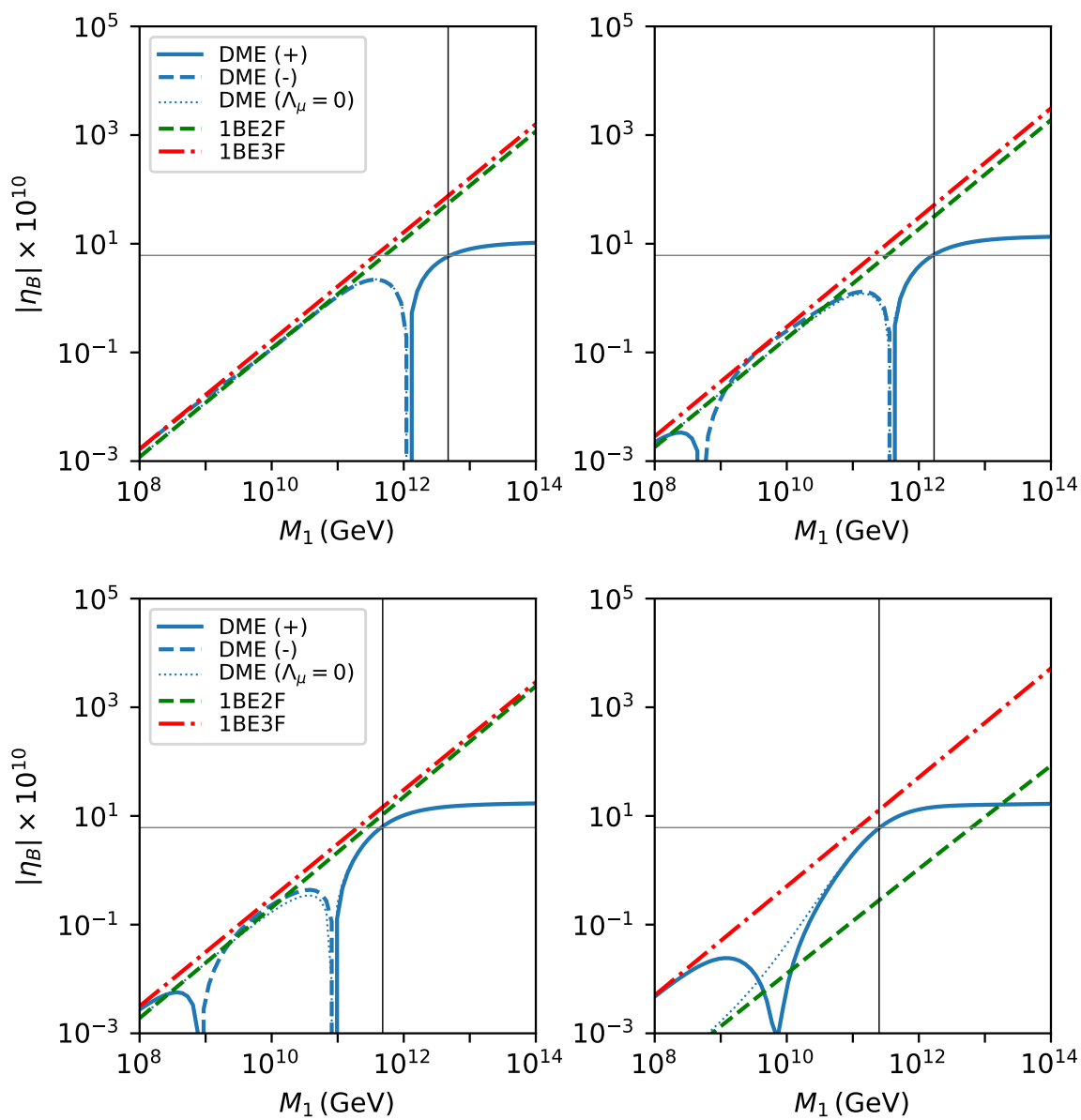

Figure 6. The four plots show the absolute value of the baryon asymmetry $\eta_{B}$ versus $M_{1}$ for $\mathrm{NH}$ spectrum, obtained with different sets of equations: DME (blue), 1BE2F (green), 1BE3F (red). The PMNS phases are chosen as in figure $5, \delta=3 \pi / 2, \alpha_{23}=0$. The top-left, top-right, bottom-left and bottom-right panels are obtained for $x=150^{\circ}, 40^{\circ}, 30^{\circ}$ and $22.2^{\circ}$, respectively. The solid (dashed) blue line corresponds to $\eta_{B}>0\left(\eta_{B}<0\right)$; the dotted blue line is obtained for $\Lambda_{\mu}=0$. The horizontal grey line marks the present BAU at $6.1 \times 10^{-10}$ and is reproduced with the solution of DMEs at the minimal mass scales marked by the vertical black line, namely at $M_{1} \simeq 4.7(1.7) \times 10^{12} \mathrm{GeV}$ top-left (top-right) panel and $4.8(2.5) \times 10^{11} \mathrm{GeV}$ bottom-left (bottom-right) panel. See text for further details.

In the bottom-right panel the two transitions overlap so that the solution to the DMEs never approaches the 1BE2F solution.

For $x=150^{\circ}$ corresponding to the top-left panel in figure 6 , successful leptogenesis takes place at $M_{1} \simeq 4.0 \times 10^{12} \mathrm{GeV}$ and the 1-to-2 flavour transition happens around $M_{1} \simeq 10^{12} \mathrm{GeV}$, as one would have expected from the considerations made after eq. (2.22). This panel shows an example of what we will call "standard" scenario of a 1-to-2 flavour transition at $\sim 10^{12} \mathrm{GeV}$ under the assumption made about the source of CP violation as well as the strong wash-out regime of $\eta_{B}$ generation. We note also that the 2-to-3 flavour transition happens at $M_{1} \sim 10^{9} \mathrm{GeV}$ with no sign change, as the DME solution (solid blue line) interpolates smoothly between the 1BE2F (green) line and the 1BE3F (red) one. 
The remaining three panels of figure 6 represent examples of "non-standard" scenarios of the transition of interest, as the 1-to-2 flavour transition happens at a mass scale which decreases from $10^{12} \mathrm{GeV}$ as $x$ decreases approaching $x^{\text {min }}$, the value of $x$ at which the sign change does not occur and, as we will discuss, $\epsilon_{\tau \tau}^{(1)}$ has an absolute maximum (bottom-right panel). The scenarios in these panels are "non-standard" for the following reasons. Firstly, the product $p_{1 \tau} p_{1 \tau^{\perp}} \simeq p_{1 \tau} \simeq 2.5 \times 10^{-2},\left(1.5 \times 10^{-3}\right)$ for $x=30^{\circ},\left(22.2^{\circ}\right)$ is so small that the integrated wash-out term in $\lambda$ is additionally strongly suppressed, allowing the plateau due the $\tau \tau^{\perp}$-decoherence contribution to extend below $10^{12} \mathrm{GeV}$. Secondly, since for $x=30^{\circ}\left(22.2^{\circ}\right)$ we have

$$
p_{1 \tau} \kappa_{1} \simeq 0.63,\left(2.8 \times 10^{-2}\right), \quad p_{1 \tau^{\perp} \kappa_{1}} \simeq \kappa_{1} \simeq 24,(19),
$$

the CPV asymmetry in the $\tau^{\perp}$-flavour is generated in the strong wash-out regime, while the CPV asymmetry in the $\tau$-flavour is produced in the weak wash-out regime. This scenario could not and was not considered in section $3 .{ }^{19}$ Finally, figure 6 shows that the two-flavour approximation in the range $10^{9} \lesssim M_{1} / \mathrm{GeV} \lesssim 10^{12}$ based on $1 \mathrm{BE} 2 \mathrm{~F}$ is not always accurate. For the case considered in the bottom-right panel of the figure, the DME solution for the asymmetry $\eta_{B}$ is enhanced by a factor of $\sim 10$ with respect to the asymmetry obtained by solving the Boltzmann equations in the two-flavour approximation. This leads, in particular, to successful leptogenesis at $M_{1} \gtrsim 2.5 \times 10^{11} \mathrm{GeV}$. Most remarkably, in the case shown in the bottom-left panel, the asymmetry $\eta_{B}$ predicted by the DMEs (blue solid curve) at $M_{1} \gtrsim 4.8 \times 10^{11} \mathrm{GeV}$ has the correct sign allowing for successful leptogenesis, while the $1 \mathrm{BE} 2 \mathrm{~F}$ solution (green curve) gives $\eta_{B}<0$ in the indicated range of $M_{1}$ and thus non-viable leptogenesis.

We note also that, as the top-right and bottom-left panels in figure 6 show, at the 2-to-3 flavour transitions at $M_{1} \cong 10^{9} \mathrm{GeV}$, the baryon asymmetry $\eta_{B}$ changes sign going through zero, in contrast to the behaviour of $\eta_{B}$ shown in the top-left and bottom-right panels. For the chosen values of the CPV phases of the PMNS matrix the presence of this zero in $\eta_{B}$, as figure 6 indicates, depends on the value of $x$. For $x=22.2^{\circ}$ (bottom-right panel), the 2-to-3 flavour transition takes place with $\eta_{B}$ not going through zero but only through a relatively shallow minimum at $M_{1} \cong 10^{10} \mathrm{GeV}$.

\section{Ranges of $M_{1}$ and $\delta$ for Viable Leptogenesis}

The case illustrated in the bottom-right panel of figure 6 is interesting for the following additional reasons. As our scan of the relevant parameter space shows, it is the case in which successful leptogenesis with two hierarchical in mass heavy Majorana neutrinos and $\mathrm{CP}$ violation provided by the Dirac phases $\delta$ takes place for the minimal for the considered scenario value of $M_{1 \text { min }} \cong 2.5 \times 10^{11} \mathrm{GeV}$. The value of $x=x^{\min }=22.2^{\circ}$ maximises the CPV asymmetry $\epsilon_{\tau \tau}^{(1)}$. Indeed, $\epsilon_{\tau \tau}^{(1)}$ depends on $x$ through the factor

$$
f_{1 \epsilon}(x)=\frac{\sqrt{a} \sin 2 x}{a \cos ^{2} x+\sin ^{2} x}, \quad a \equiv m_{2} / m_{3},
$$

\footnotetext{
${ }^{19}$ Moreover, since $p_{1 \tau} \kappa_{1} \gtrsim 10^{-2}$, the analytic approximation used in section 3 in the weak wash-out regime is not sufficiently accurate [64].
} 
which has an absolute maximum $\mathrm{at}^{20} x^{\mathrm{min}}=22.2^{\circ}: f_{\epsilon}\left(x^{\mathrm{min}}\right) \cong 1.00$. The chosen value of $\delta$ also maximises $\left|\epsilon_{\tau \tau}^{(1)}\right|$. As $M_{1}$ increases from the value $M_{1} \cong 2.5 \times 10^{11} \mathrm{GeV}, \eta_{B}$ also increases from $\eta_{B}=6.1 \times 10^{-10}$ and, as figure 6 bottom-right panel shows, for $x=x^{\text {min }}$ and $\delta=3 \pi / 2$ reaches a plateau at $M_{1}=2.7 \times 10^{12} \mathrm{GeV}$, where $\eta_{B}=1.60 \times 10^{-9}$ and $^{21}$ is larger than the observed value of $\eta_{B}$ by the factor $C_{P 1} \cong 2.62$. For the value of $M_{1} \gtrsim$ $2.7 \times 10^{12} \mathrm{GeV}$ of the plateau, we have $\eta_{B} \propto\left(-\epsilon_{\tau \tau}^{(1)}\right)$ (see eq. (4.17)). Thus, $\eta_{B}$ will be compatible with the observed value of BAU for smaller value of $\left(-\epsilon_{\tau \tau}^{(1)}\right)>0$, i.e., for smaller $\left(-f_{\epsilon}(x) \sin \delta\right)>0$. The plateau value of $\eta_{B}$ corresponds to $x=x^{\min }$ and $\delta=3 \pi / 2$ for which $\left(-f_{\epsilon}\left(x^{\min } \sin (3 \pi / 2)\right)=1\right.$. Thus, fixing $x=x^{\text {min }}$ we get the minimal value of $(-\sin \delta)>0$ for which we can have successful leptogenesis at $M_{1} \gtrsim 2.7 \times 10^{12} \mathrm{GeV}$ :

$$
(-\sin \delta) \gtrsim C_{P 1}^{-1} \cong 0.38, \text { or } 202.4^{\circ} \lesssim \delta \lesssim 337.6^{\circ}
$$

The derived condition on $\delta$ is a necessary condition for successful leptogenesis within the considered scenario. ${ }^{22}$

As $M_{1}$ decreases from $2.7 \times 10^{12} \mathrm{GeV}$ to $2.5 \times 10^{11} \mathrm{GeV}, \eta_{B}$ decreases from the value at the plateau to the observed value and correspondingly, the interval of values of $\delta$ for which one can have viable leptogenesis also decreases shrinking to the point $\delta=3 \pi / 2$ at $M_{1}=2.5 \times 10^{11}$. Clearly, there exists a correlation between the value of $\delta$ and the scale $M_{1}$ of viable leptogenesis. It follows from the preceding discussion also that in the considered scenario of CP violation provided by the Dirac CPV phase $\delta$ of the PMNS matrix, it is possible to reproduce the observed value of BAU for values of $M_{1}$ spanning at least three orders of magnitude, i.e., for $2.5 \times 10^{11} \lesssim M_{1} / \mathrm{GeV} \lesssim 10^{14}$.

In the case we have considered with $\alpha_{23}=0$ and $x=x^{\min }=22.2^{\circ}$, we can have successful leptogenesis, as eq. (4.20) shows, for $\delta$ lying in the interval $\pi<\delta<2 \pi$, where $\sin \delta<0$. So, the sign of $\sin \delta$ is anticorrelated with the sign of the observed $\eta_{B}$. This result holds also for the alternative possible values of $\alpha_{23}= \pm 2 \pi$ and all possible value of $x$, for which we can have viable leptogenesis. In other words, in the case under study there are no values of $\delta$ from the interval $0<\delta<\pi$ where $\sin \delta>0$, for which it is possible to reproduce the observed value of BAU.

Indeed, we note first that there is a periodicity of $\pi$ in the dependence on $x$, and of $4 \pi$ in the dependence on $\alpha_{23}$, of all relevant quantities on which the predicted sign of $\eta_{B}$ depends: $\epsilon_{\tau \tau}^{(1)}, k_{1}$ and $p_{1 \tau}$ (see eqs. $(4.3)-(4.12)$ ). Therefore one gets the same results for

\footnotetext{
${ }^{20}$ To be more precise, $f_{\epsilon}(x)$ has an absolute maximum at $x^{\max }=0.5 \arccos \left(\left(m_{3}-m_{2}\right) /\left(m_{3}+m_{2}\right)\right)=22.5^{\circ}$, where we have made use of $m_{3}=\sqrt{\Delta m_{31}^{2}}, m_{2}=\sqrt{\Delta m_{21}^{2}}$ and the best-fit values of $\Delta m_{31}^{2}$ and $\Delta m_{21}^{2}$ given in table 1 . However, as can be easily checked, $f_{\epsilon}\left(x^{\max }\right)-f_{\epsilon}\left(x^{\mathrm{min}}\right) \cong 10^{-4}$.

${ }^{21}$ As $M_{1}$ increases beyond $2.7 \times 10^{12} \mathrm{GeV}, \eta_{B}$ continues to grow very slowly due to the dependence of $\epsilon_{\tau \tau}^{(1)}$ on $f^{-1}\left(M_{2}\right) / M_{2}$, and at $M_{1}=10^{14} \mathrm{GeV}\left(M_{2}=10 M_{1}\right)$ we have $\eta_{B} \cong 1.67 \times 10^{-9}$.

${ }^{22}$ In [18] in the same scenario the following condition for successful leptogeneis was obtained using the $1 \mathrm{BE} 2 \mathrm{~F}$ and assuming that the two-flavoured leptogenesis regime does not extend beyond $M_{1}=5 \times 10^{11} \mathrm{GeV}$ : $\left|\sin \theta_{13} \sin \delta\right| \gtrsim 0.090$. In the same article the minimal scale of viable leptogenesis was found to be $M_{1 \mathrm{~min}} \cong$ $2.2 \times 10^{11} \mathrm{GeV}$, to be compared with $M_{1 \mathrm{~min}} \cong 2.5 \times 10^{11} \mathrm{GeV}$ found by us. The lower limit on $(-\sin \delta)$ we have obtained in eq. (4.20) implies $\left|\sin \theta_{13} \sin \delta\right| \gtrsim 0.057$, where we have used the best fit value of $\theta_{13}$ from table 1. It is clear that our results based on the DME, in particular, extend the ranges of $\delta$ and $M_{1}$, for which we can have successful leptogenesis, derived in [18].
} 

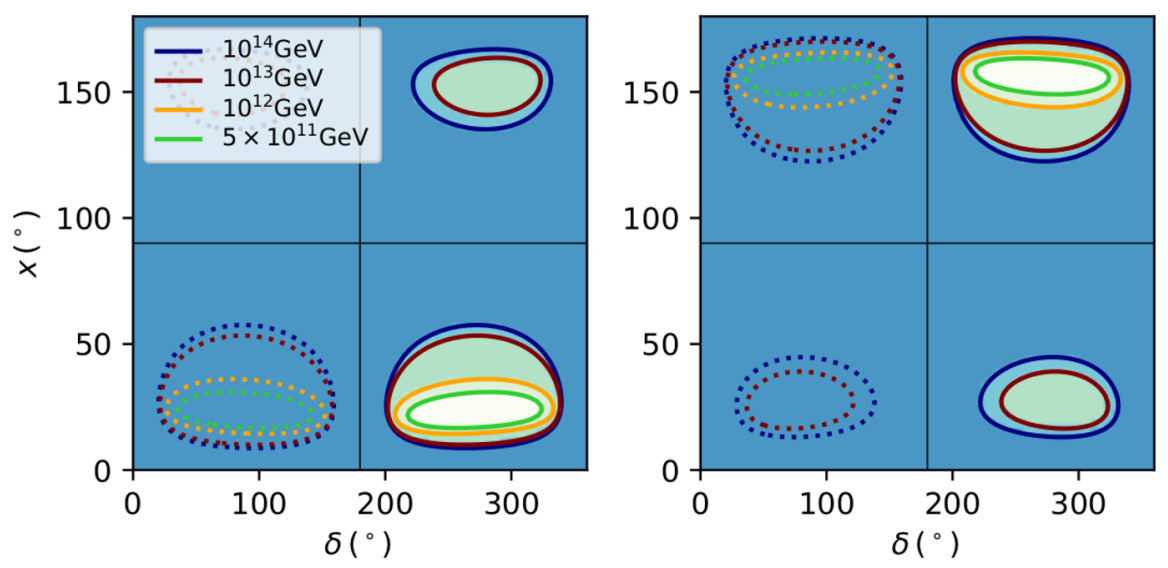

Figure 7. Regions of viable leptogenesis in the $\delta-x$ half plane, $0 \leq x \leq \pi$, for NH spectrum, real $R$-matrix, CP violation due to the Dirac phase $\delta, \alpha_{23}=0$ (left panel) and $2 \pi$ (right panel) and different values of $M_{1}$. The solid contours corresponding to fixed values of $M_{1}$ surround the regions in which there is a combination of values of $\delta$ and $x$ for which $\eta_{B}=6.1 \times 10^{-10}$. The dotted contours surround regions where one can have $\left|\eta_{B}\right|=6.1 \times 10^{-10}$ but $\eta_{B}<0$. The predicted $\eta_{B}$ outside the contours is always smaller in magnitude than the observed BAU. The regions of viable leptogenesis in the half-plane $-\pi \leq x \leq 0$ (or $\pi \leq x \leq 2 \pi$ ), which are not shown, can be obtained from those in the figure by substituting $x$ with $x-\pi$. See text for further details.

$x$ and $x-\pi$. In the example with $\alpha_{23}=0$ and $x=22.2^{\circ}$ we have considered, we get the same result for $x=-157.8^{\circ}$ (or equivalently $x=202.2^{\circ}$ ). If we set $\alpha_{23}=2 \pi$, the results will be the same for $\alpha_{23}=-2 \pi$. Therefore the only possibility to have viable leptogenesis with $\sin \delta>0$ is when $\alpha_{23}=2 \pi$. The quantities $\Re\left(U_{\tau 2}^{*} U_{\tau 3}\right) \sin 2 x \propto-\beta_{\alpha} \sin 2 x$ and $\Im\left(U_{\tau 2}^{*} U_{\tau 3}\right) \sin 2 x \propto \beta_{\alpha} \sin \delta \sin 2 x$, on which respectively $p_{1 \tau}$ and $\epsilon_{\tau \tau}^{(1)}$ depend, change sign when $\alpha_{23}$ is changed from 0 to $2 \pi$ : $\beta_{\alpha}=1(-1)$ for $\alpha_{23}=0(2 \pi)$. In addition $\Re\left(U_{\tau 2}^{*} U_{\tau 3}\right)$ and $p_{1 \tau}$ exhibit a sub-leading dependence on $\delta$ via terms proportional to $\sin \theta_{13} \cos \delta$. Thus, in what concerns the present discussion, changing the sign of $\sin \delta$ has negligible effect on $p_{1 \tau}$. We recall that for $\alpha_{23}=0, p_{1 \tau}$ has a minimum at $x^{\text {min }}=22.2^{\circ}$ where $p_{1 \tau} \ll 1$, so that we have $\left(1-2 p_{1 \tau}\right)>0$ for the quantity on which, in particular, the sign of $\eta_{B}$ depends. The change of the sign of $\Re\left(U_{\tau 2}^{*} U_{\tau 3}\right) \sin 2 x$ leads to a significant change of the value of $p_{1 \tau}$, leading for $x^{\mathrm{min}}=22.2^{\circ}$ to $\left(1-2 p_{1 \tau}\right)<0$ and thus to non-viable leptogenesis for $\sin \delta>0$.

It follows from the preceding considerations that the change of the signs of both $\Re\left(U_{\tau 2}^{*} U_{\tau 3}\right)$ and $\Im\left(U_{\tau 2}^{*} U_{\tau 3}\right)$ in the expressions for $p_{1 \tau}$ and $\epsilon_{\tau \tau}^{(1)}$ can only be compensated simultaneously by changing $x$ to $\pi-x$, i.e., by a change of the sign of $\sin 2 x$. This implies that in addition to the solutions we have found for $\alpha_{23}=0$ for certain ranges of $x$ (e.g., for $0<x<\pi / 2)$ and of $\delta$ in the interval $\pi<\delta<2 \pi$, for $\alpha_{23}=2 \pi$ we will have successful leptogenesis in the range of $\pi-x$ (e.g., for $\pi / 2<x<\pi$ ) and for $\delta$ in the same interval. Thus, in the case of $\alpha_{23}=2 \pi$, a value of $\delta$ from the interval $0<\delta<\pi$ with $\sin 2 x<0$ $(\sin 2 x>0)$ leads either to a wrong sign of $\eta_{B}$ due to the interplay of the signs of $p_{1 \tau}$ and $\epsilon_{\tau \tau}^{(1)}$, or else to a value of $\eta_{B}$ which is smaller than the observed one. 


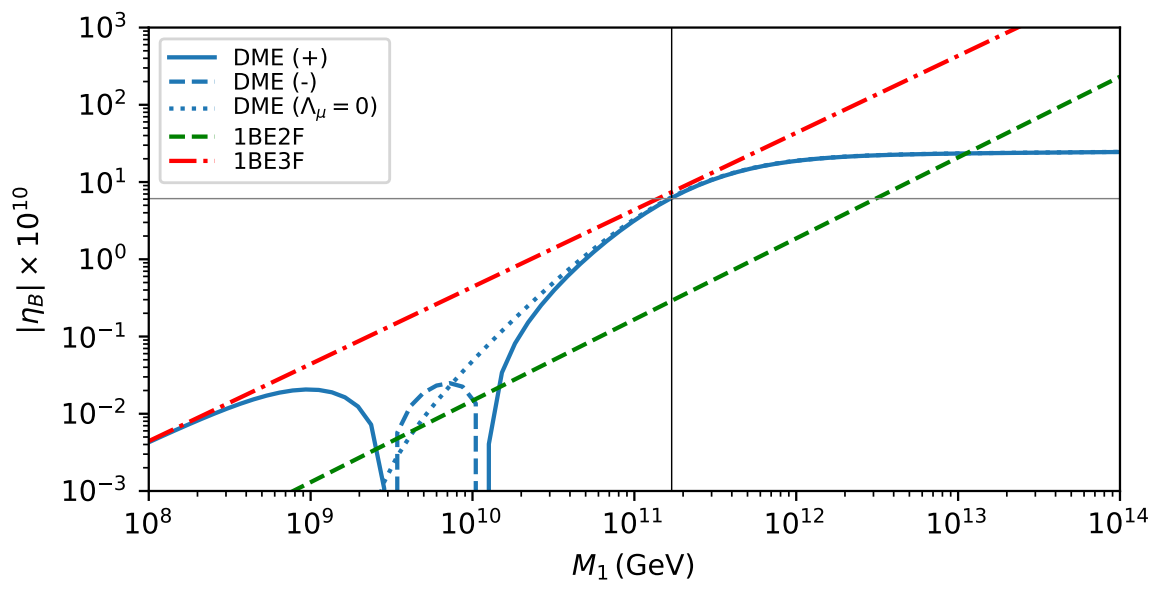

Figure 8. The baryon asymmetry $\eta_{B}$ versus $M_{1}$ for $\mathrm{NH}$ spectrum, $x=0, y=26^{\circ}\left(-26^{\circ}\right)$, $\delta=\pi / 2$ and $\alpha_{23}=\pi(3 \pi)$, for which successful leptogenesis takes place for the minimal value of $M_{1} \cong 1.7 \times 10^{11} \mathrm{GeV}$ (vertical black line). The requisite $\mathrm{CP}$ violation is provided by the Dirac phase $\delta$. The horizontal black line corresponds to the observed $\eta_{B}=6.1 \times 10^{-10}$. See text for further details.

The conclusions of the preceding discussions are confirmed by the numerical scan of the parameters $\delta$ and $x$ in the case of $\alpha_{23}=0$ and $2 \pi$ and several fixed values of $M_{1}$, the results of which are shown in figure 7 . Thus, in the considered scenario there is a direct and unique relation between the sign of $\sin \delta$ and the sign of the baryon asymmetry of the Universe. If the measurement of $\delta$ in the low-energy neutrino oscillation experiments will show that $\delta$ lies in the interval $[0, \pi]$, the considered leptogenesis scenario will be ruled out. If, however, $\delta$ will be found to lie in the lower half-plane, $\pi<\delta<2 \pi$, this will not only lend support for the discussed scenario, but also will allow to obtain constraints on the leptogenesis scale.

Given that for $x \neq 0, y=0$ and CP violation due only to the Dirac phase $\delta$ leptogenesis is unsuccessful at any mass scale in the IH case (see eq. (4.13) and the discussion related to it) we do not consider this case.

\subsubsection{Purely imaginary $R_{11} R_{12}\left(R_{12} R_{13}\right)(x=k \pi, k=0,1,2, y \neq 0)$}

We discuss next the leptogenesis scenario in which $\mathrm{CP}$ violation is still provided by the Dirac phase only, but now $x=k \pi, k=0,1,2$, and $y \neq 0$ so that the product $R_{12} R_{13}\left(R_{11} R_{12}\right)$ is purely imaginary in the $\mathrm{NH}(\mathrm{IH})$ case and the suppression of the CPV asymmetry shown in eq. (4.13) is avoided.

NH spectrum. The analysis is similar to that performed in the preceding subsection. We report below the results on the ranges of $\delta$ and $M_{1}$ for which one can have successful leptogenesis. For the minimal value of $M_{1}$ we get $M_{1}=1.7 \times 10^{11} \mathrm{GeV}$, which is obtained for $\delta=\pi / 2, y=26^{\circ}\left(y=-26^{\circ}\right)$ and $\alpha_{23}=\pi$ or $-3 \pi(3 \pi$ or $-\pi)$. This case is illustrated 


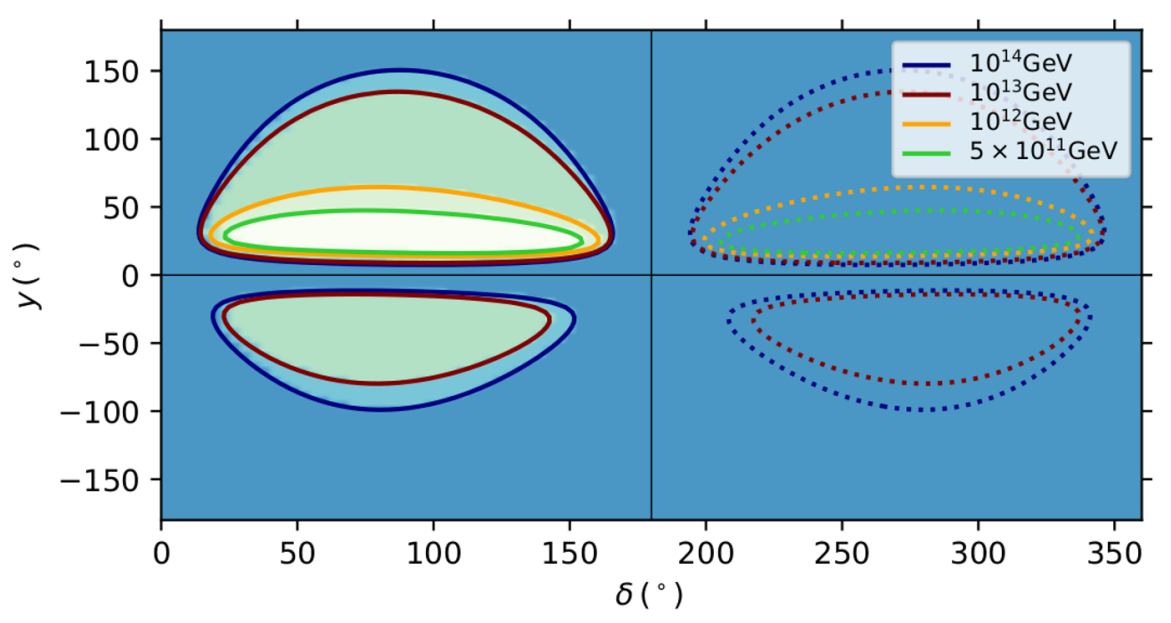

Figure 9. Regions of viable leptogenesis in the $\delta-y$ plane for NH spectrum, $x=k \pi, k=0,1,2$, $\alpha_{23}=\pi$ and different $M_{1}$. The solid contours corresponding to fixed values of $M_{1}$ surround the regions in which there is a combination of values of $\delta$ and $y$ for which $\eta_{B}=6.1 \times 10^{-10}$. The dotted contours surround regions where one can have $\left|\eta_{B}\right|=6.1 \times 10^{-10}$ but $\eta_{B}<0$. The predicted $\eta_{B}$ outside the contours is smaller in magnitude than the present BAU. Setting $\alpha_{23}=3 \pi$ leads to a figure which can be obtained from the present by changing $y$ to $-y$. See text for further details.

by figure 8 . The value of $y$ maximises the factor

$$
f_{2 \epsilon}(x)=\frac{\sqrt{a} \sinh 2 y}{a \cosh ^{2} y+\sinh ^{2} y}, \quad a \equiv m_{2} / m_{3},
$$

in the expression for $\epsilon_{\tau \tau}^{(1)}$, and thus maximises $\epsilon_{\tau \tau}^{(1)}$ with respect to $y$. For $\alpha_{23}=\pi$ or $(-3 \pi)$ the value of $\delta=\pi / 2$ maximises $\epsilon_{\tau \tau}^{(1)}$ which is proportional to $\sin \delta$. At the plateau which begins at $M_{1} \cong 2.1 \times 10^{12} \mathrm{GeV}$ we have $\eta_{B} \cong C_{P 2} 6.1 \times 10^{-10}$ with $C_{P 2} \cong 3.9$. Correspondingly, at $M_{1} \gtrsim 2.1 \times 10^{12} \mathrm{GeV}$ we can have successful leptogenesis for

$$
\sin \delta \gtrsim C_{P 2}^{-1} \cong 0.25, \text { or } 14.6^{\circ} \lesssim \delta \lesssim 165.4^{\circ} \text {, }
$$

As $M_{1}$ decreases from $2.1 \times 10^{12} \mathrm{GeV}$ to $1.7 \times 10^{11} \mathrm{GeV}, \eta_{B}$ decreases from the value at the plateau to the observed value and the width of the intervals of values of $\delta$ in eq. (4.22) decreases. At $M_{1}=1.7 \times 10^{11} \mathrm{GeV}$ it shrinks to the point $\delta=\pi / 2$. In what concerns $M_{1}$, successful leptogenesis is possible for values of $M_{1} \gtrsim 1.7 \times 10^{11} \mathrm{GeV}$, which span at least three orders of magnitude.

It follows from the preceding discussion that for $\alpha_{23}=\pi$ or $(-3 \pi)$, one can have successful leptogenesis for value of $\delta$ from the interval $0<\delta<\pi$ where $\sin \delta>0$. Performing an analysis similar to that in the preceding subsection, we find that also in this case there is a direct relation between the sign of $\sin \delta$ and the sign of the observed BAU in the sense that for the values of the parameters in the considered case, no region with viable leptogenesis exists for $\delta$ from the interval $\pi<\delta<2 \pi$, where $\sin \delta<0$. Changing the value of $\alpha_{23}$ from $\pi$ to $3 \pi$, for example, one finds that the viable regions of values of $y$ and $\delta$ from the interval $0<\delta<\pi$, for which it is possible to reproduce the observed value of BAU, shift to 
the regions corresponding to $(-y)$ with $\delta$ remaining in the same interval $0<\delta<\pi$. This is confirmed by the numerical scan of the $y-\delta$ parameter space for $\alpha_{23}=\pi$ and $3 \pi$, the results of which are shown in figure 9 .

Obviously, the discussed leptogenesis scenario will be ruled out if $\delta$ determined in neutrino oscillation experiments is found definitely to lie in the interval $[\pi, 2 \pi]$, while if $\delta$ is found to be in the upper half-plane, $0<\delta<\pi$, the scenario will be proven viable and it will be possible to obtain also constraints on the leptogenesis scale of the scenario.

IH spectrum. We analyse in somewhat greater detail the case of IH spectrum. We show in figure 10 the modulus of the baryon asymmetry $\eta_{B}$ versus $M_{1}$ for the IH spectrum with $\delta=3 \pi / 2, \alpha_{21}=\pi, y=-100^{\circ}$ (left panel) and $y=-46.5^{\circ}$ (right panel). The dependence of $\eta_{B}$ on $M_{1}$ exhibits a number of interesting features. The 1-to-2 flavour transition described by DME takes place with a sign change of $\eta_{B}$. At values of $M_{1}<M_{10}\left(M_{1}>M_{10}\right), M_{10}$ being the value of $M_{1}$ at which $\eta_{B}=0$, we have $\eta_{B}<0\left(\eta_{B}>0\right)$. In the case illustrated in figure 10 , we have $M_{10} \ll 10^{12} \mathrm{GeV}$. When $y$ changes from $\left(-100^{\circ}\right)$ to $\left(-46.5^{\circ}\right), M_{10}$ decreases from $6.0 \times 10^{10} \mathrm{GeV}$ to $2.4 \times 10^{10} \mathrm{GeV}$. Most importantly, the minimal value of $M_{1}$ at which one can have successful leptogenesis also decreases from $M_{1}=1.6 \times 10^{11} \mathrm{GeV}$ to $M_{1}=6.2 \times 10^{10}$, with both values being $\ll 10^{12} \mathrm{GeV}$.

Further, the DME solution for $\eta_{B}$ shown in the left (right) panel of figure 10 is at $M_{1} \leq 10^{12} \mathrm{GeV}$ larger than (similar in magnitude to) $\left|\eta_{B}\right|$ found with $1 \mathrm{BE} 2 \mathrm{~F}$, except in a narrow region around $M_{10}$. Still, $\eta_{B}$ obtained from the $1 \mathrm{BE} 2 \mathrm{~F}$ equations shown in both panels, in contrast to that derived from DME ones, has a wrong sign, i.e., predicts $\eta_{B}<0$ and thus non-viable leptogenesis. The value of $\left|\eta_{B}\right|$ obtained with 1BE3F is in both cases, as the panels show, significantly smaller than those found with DME and 1BE2F. Moreover, the 2-to-3 flavour transition described by the DME solution takes place at $M_{1} \lesssim 10^{8} \mathrm{GeV}$, with the $\mu$-Yukawa interaction having the effect of enhancing the DME solution for $\left|\eta_{B}\right|$ in the interval $10^{8} \lesssim M_{1} / \mathrm{GeV} \lesssim 10^{10}$. Both these features are in the region of values of $M_{1}$ for which the calculated $\left|\eta_{B}\right|$ is significantly smaller that the observed $\eta_{B}$. However, they might be relevant in a leptogenesis scenario with three heavy Majorana neutrinos with non-hierarchical masses, e.g., with $M_{3} \cong 3 M_{2} \cong 9 M_{1}$.

In what concerns the range of $\delta$ and $M_{1}$ for which we have successful leptogenesis, we find that (see figure 11): i) the minimal value of $M_{1}$ is $M_{1} \cong 4.6 \times 10^{10} \mathrm{GeV}$ and corresponds to the values of $\alpha_{21}=\pi(3 \pi), y=-73^{\circ}\left(y=+73^{\circ}\right)$ and $\delta=211^{\circ}$; ii) the plateau of values of $\eta_{B}$ is present at $M_{1} \gtrsim 1.2 \times 10^{12} \mathrm{GeV}$; iii) at the plateau $\eta_{B} \cong C_{P 3} 6.1 \times 10^{-10}$ with $C_{P 3} \cong 6.1$. Correspondingly, at $M_{1} \gtrsim 1.2 \times 10^{12} \mathrm{GeV}$ successful leptogenesis is possible for

$$
|\sin \delta| \gtrsim C_{P 3}^{-1}\left|\sin \left(\delta=211^{\circ}\right)\right| \cong 0.084, \text { or } 185^{\circ} \lesssim \delta \lesssim 355^{\circ},
$$

where we have used the fact that the plateau value of $\eta_{B}$ corresponds to $\delta=211^{\circ}$.

We note that for the chosen values of $\alpha_{21}=\pi, y=-73^{\circ}, \epsilon_{\tau \tau}^{(1)}$ is proportional to $\sin \delta$ and thus at $\delta=211^{\circ}\left|\epsilon_{\tau \tau}^{(1)}\right|$ is smaller by the factor 0.515 than for $\delta=3 \pi / 2$. However, due to the fact that, as can be shown, the value of $p_{1 \tau}$ at $\delta=211^{\circ}$ is smaller approximately by a factor of 6 than that at $\delta=3 \pi / 2$, the minimal $M_{1}$ at which we can have successful leptogenesis is also smaller than the one for $3 \pi / 2$ which reads $M_{1} \cong 10^{11} \mathrm{GeV}$. At the same 

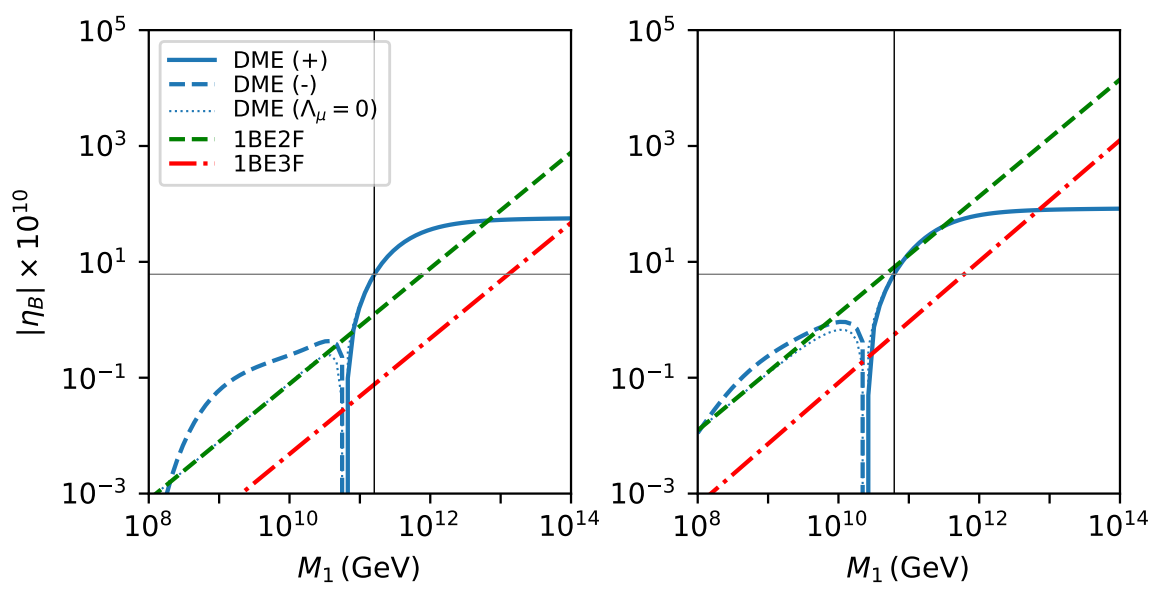

Figure 10. The same as in figure 6 but for $x=0$ and $y \neq 0$ and in the IH case. The two panels correspond to $\mathrm{CP}$ violation from the Dirac phase only with $\delta=3 \pi / 2, \alpha_{21}=\pi, y=-100^{\circ}$ (left panel) and $y=-46.5^{\circ}$ (right panel). The vertical black lines correspond to $M_{1} \simeq 1.6 \times 10^{11} \mathrm{GeV}$ (left panel) and $M_{1} \simeq 6.2 \times 10^{10} \mathrm{GeV}$ (right panel). See text for further details.

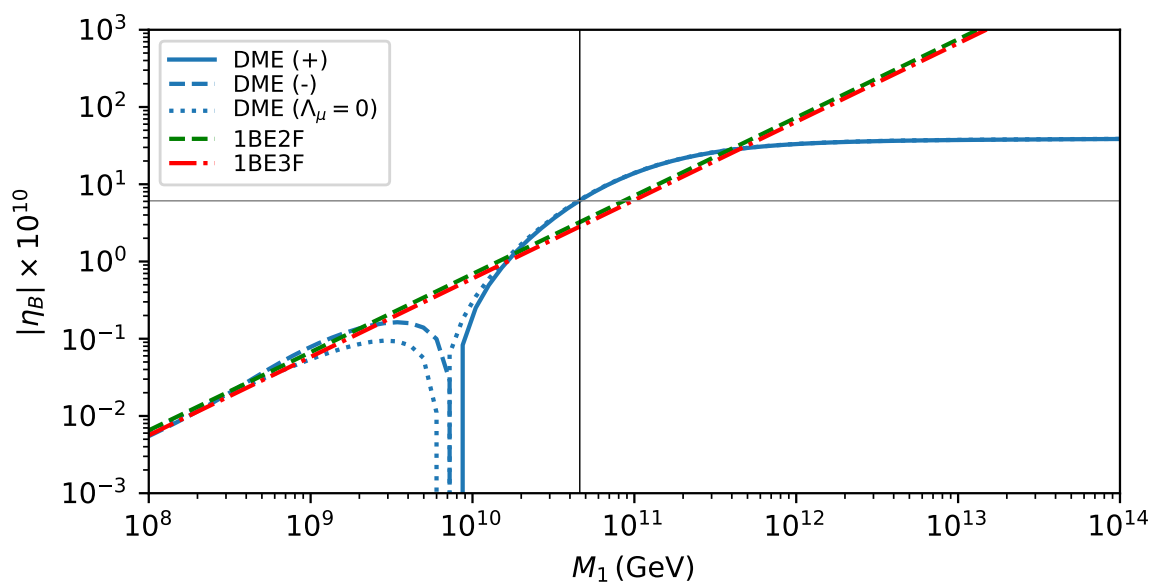

Figure 11. The baryon asymmetry $\eta_{B}$ versus $M_{1}$ for IH spectrum, $x=0, \pi, y=-73^{\circ}\left(y=73^{\circ}\right)$, $\alpha_{21}=\pi(3 \pi)$ and $\delta=211^{\circ}$, for which successful leptogenesis takes place for the minimal value of $M_{1} \cong 4.6 \times 10^{10} \mathrm{GeV}$ (vertical black line). The requisite $\mathrm{CP}$ violation is provided by the Dirac phase $\delta$. The horizontal black line corresponds to the observed $\eta_{B}=6.1 \times 10^{-10}$. See text for further details.

time, $\eta_{B}$ at the plateau for $\delta=3 \pi / 2$ is by a factor of approximately 1.9 times larger than the plateau value of $\eta_{B}$ for $\delta=211^{\circ}$ and reads: $\eta_{B}=7.3 \times 10^{-9}$.

As we have seen, in the discussed case of Dirac CP violation and IH neutrino mass spectrum, successful leptogenesis is possible for values of $\delta$ from the interval $\pi<\delta<2 \pi$ where $\sin \delta<0$. Performing a scan over $y$ and $\delta$ for the possible values of $\alpha_{21}=\pi$ and $3 \pi$ with $x=k \pi, k=0,1,2$, we find that for $M_{1} \gtrsim 10^{13} \mathrm{GeV}$ one can have a successful leptogenesis also for values of $\delta$ from the interval $0<\delta<\pi$, and a small range of values of $y$ from the interval $0<y<50^{\circ}$. The appearance of this second region is related to the slow increase of $\epsilon_{\tau \tau}^{(1)}$ and thus of $\eta_{B}$ with $M_{1}$ due to the factor $f^{-1}\left(M_{2}\right) / M_{2}$. The results of 


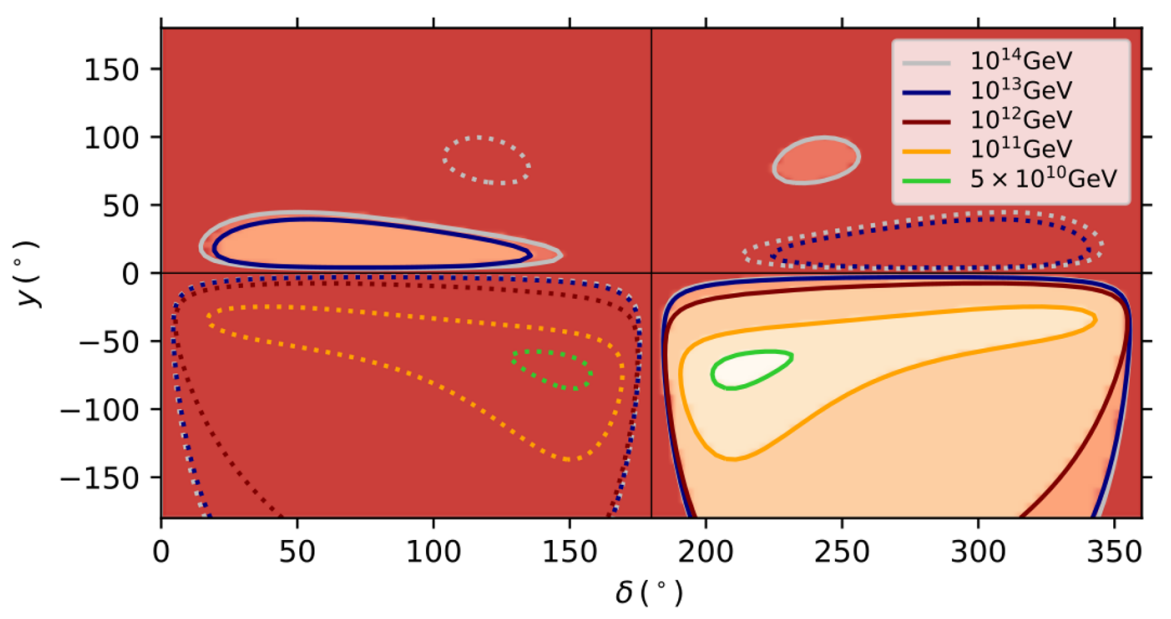

Figure 12. Regions of viable leptogenesis in the $\delta-y$ plane for IH spectrum, $x=k \pi, k=$ $0,1,2, \alpha_{21}=\pi$, corresponding to $\mathrm{CP}$ violation due to the Dirac phase $\delta$, and different $M_{1}$. The solid contours corresponding to fixed values of $M_{1}$ surround the regions in which there exists a combination of values of $\delta$ and $y$ for which $\eta_{B}=6.1 \times 10^{-10}$. The dotted contours surround regions where one can have $\left|\eta_{B}\right|=6.1 \times 10^{-10}$ but $\eta_{B}<0$. The predicted $\eta_{B}$ outside the contours is smaller in magnitude than the present BAU. Setting $\alpha_{21}=3 \pi$ leads to a figure which can be obtained from the present by changing $y$ to $-y$. See text for further details.

the scan are presented graphically in figure 12. Thus, in this case we have a direct relation between the sign of $\sin \delta$ and the sign of the baryon asymmetry of the Universe only for $M_{1}<10^{13} \mathrm{GeV}$.

\subsection{CP violation due to the Majorana phases}

We investigate next the considered leptogenesis scenario with two heavy Majorana neutrinos with hierarchical masses in which the CP violation is provided only by the Majorana phases of the PMNS matrix. Thus, the Dirac phase and the $R$-matrix elements are chosen not to contribute to the $\mathrm{CP}$ violation necessary for the generation of BAU. We note that in the case of $\mathrm{CP}$ violation due to the Majorana phases, the additional CP violation due to the Dirac phase has sub-leading effects in leptogenesis as a consequence of the suppression by the factor $\sin \theta_{13}$. However, in certain cases these effects are non-negligible.

NH spectrum. For real $R$-matrix it is impossible to have successful leptogenesis for IH light neutrino mass spectrum and $\mathrm{CP}$ violation originating from CPV phases of the PMNS matrix, as we have already discussed, so we consider only the case of $\mathrm{NH}$ spectrum. We show in figure 13, top-left (top-right) panel, example of the behaviour of $\eta_{B}$ as a function of $M_{1}$ for $y=0$ (real $R$-matrix), $x=30^{\circ}\left(20.4^{\circ}\right), \delta=\pi$, and $\alpha_{23}=3 \pi / 2\left(700^{\circ}\right)$. The vertical black lines are at $M_{1} \simeq 2.7 \times 10^{12} \mathrm{GeV}$ (left panel) and $M_{1} \simeq 1.7 \times 10^{11} \mathrm{GeV}$ (right panel) and intersect the horizontal grey line of the observed BAU at the points of successful leptogenesis. The scenarios illustrated in the top-left and top-right panels of figure 13 correspond respectively to what we dubbed "standard" and "non-standard" behaviour of the baryon asymmetry $\eta_{B}$. The salient features of the behaviour of $\eta_{B}$ in the two cases are analogous to those discussed in detail in the two preceding subsections and we are not going to comment on them further. 

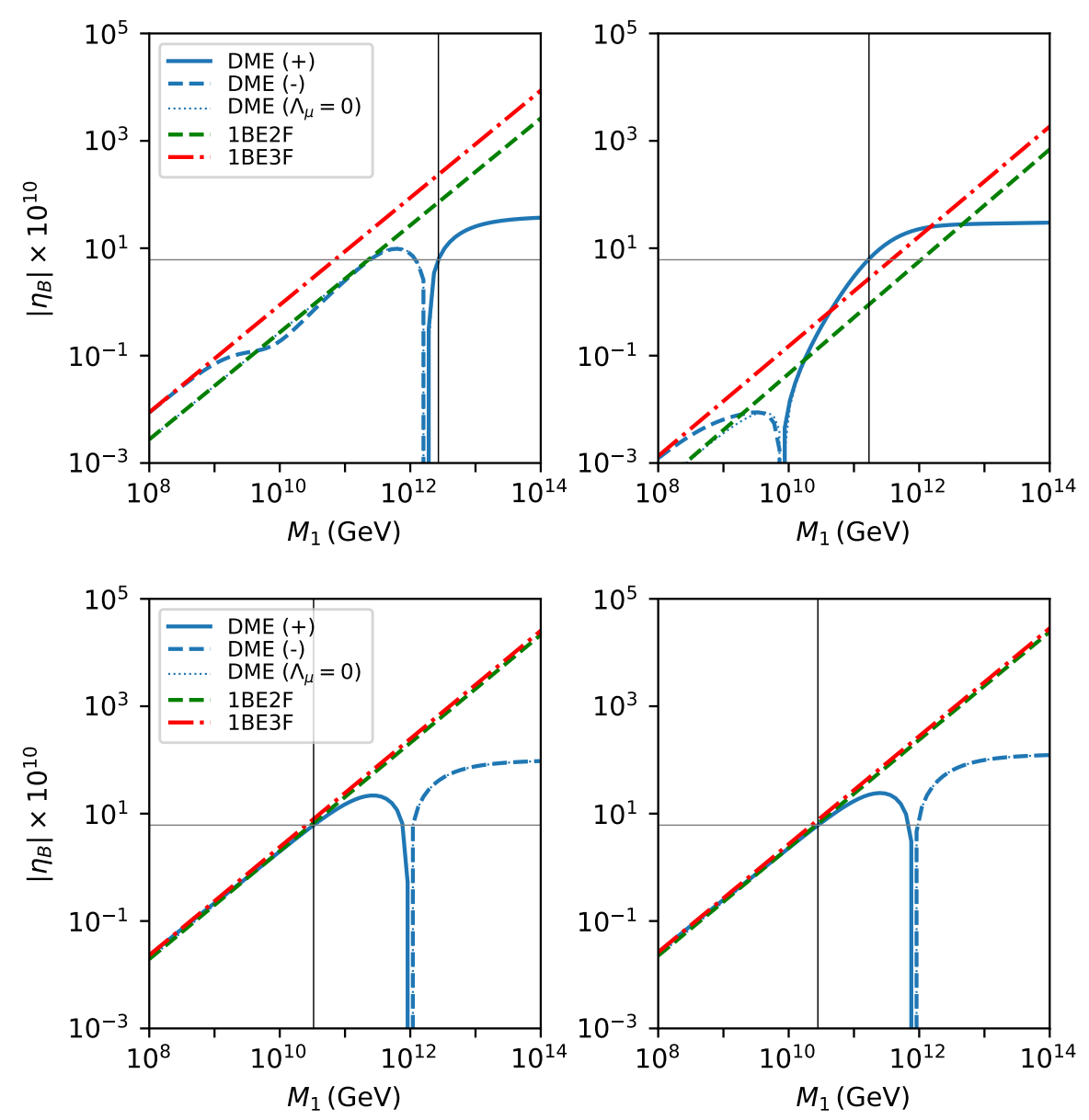

Figure 13. Leptogenesis with CP violation due to the Majorana phases of the PMNS matrix in the case of NH spectrum. Top panels: Two examples of "standard" (left) and "non-standard" (right) behaviour of $\eta_{B}$ as a function of $M_{1}$. The results shown are obtained as those in figure 6 . The parameters in the left (right) panel are set to $\delta=\pi, y=0, x=30^{\circ}\left(20.4^{\circ}\right)$ and $\alpha_{23}=3 \pi / 2\left(700^{\circ}\right)$. The vertical black lines are at $M_{1} \simeq 2.7 \times 10^{12} \mathrm{GeV}$ (left) and $M_{1} \simeq 1.7 \times 10^{11} \mathrm{GeV}$ (right) and intersect the horizontal grey line of the observed BAU at the points of successful leptogenesis. Bottom panels: The left (right) panel show the dependence of $\eta_{B}$ on $M_{1}$ for real (purely imaginary) $R_{12} R_{13}$ and $\delta=\pi$ in the case of minimal $M_{1}$ for which it is possible to have successful leptogenesis with $\mathrm{CP}$ violation provided by the Majorana phase $\alpha_{23}$ : the left (right) panel are obtained for $x=20^{\circ}, y=0\left(x=k \pi, k=0,1,2, y=19^{\circ}\right)$ and $\alpha_{23}=102^{\circ}\left(80^{\circ}\right)$. See text for further details.

We show in the bottom-left (right) panel of figure 13 the dependence of $\eta_{B}$ on $M_{1}$ for real (purely imaginary) $R_{12} R_{13}$ and $\delta=\pi$ in the case in which it is possible to have successful leptogenesis with $\mathrm{CP}$ violation provided by the Majorana phase $\alpha_{23}$ for the minimal in the considered scenario value of $M_{1}$. The other relevant parameters have the following values in the left (right) panels: $x=20^{\circ}, y=0\left(x=k \pi, k=0,1,2, y=19^{\circ}\right)$ and $\alpha_{23}=102^{\circ}\left(80^{\circ}\right)$. We will discuss in some detail in what follows first the case of $x=20^{\circ}$, $y=0$ and $\alpha 23=102^{\circ}$, extending the discussion after that to the whole plane $0<x<180^{\circ}$. 
The bottom-left panel of figure 13 illustrates one example of ranges of values of the Majorana phase $\alpha_{23}$ and $M_{1}$, for which one can have successful leptogenesis in the case of $0<x<90^{\circ}, y=0, \delta=\pi$ and $\mathrm{CP}$ violation provided by $\alpha_{23}$. As we have already remarked, this example corresponds to minimal $M_{1}$ for having viable leptogenesis in the case under study and is by far not exhaustive. We identify next qualitatively all the regions of $\alpha_{23}$ in the interval $0<\alpha_{23}<720^{\circ}$ and of $M_{1}$ where it is possible to have viable leptogenesis with $0<x<90^{\circ}, y=0$ and $\delta=\pi$.

We note first that in the case under discussion $\eta_{B}$ goes through zero and changes sign in the 1-to-2 flavour regime transition in any of the regions of the parameter space of interest and there is always a plateau of values of $\left|\eta_{B}\right|$ at $M_{1}>M_{10}, M_{10}$ being the value of $M_{1}$ at which $\eta_{B}=0$. We recall that $\operatorname{sgn}\left(\eta_{B}\right)$ at the plateau is determined by $\operatorname{sgn}\left(-\left(1-2 p_{1 \tau}\right) \epsilon_{\tau \tau}^{(1)}\right)$ (see eq. (4.17)), where $\operatorname{sgn}\left(\epsilon_{\tau \tau}^{(1)}\right)=\operatorname{sgn}\left(\Im\left(U_{\tau 2}^{*} U_{\tau 3}\right) \sin 2 x\right)$. It is not difficult to check that for the best-fit values of the neutrino oscillation parameters $\theta_{12}, \theta_{13}, \theta_{23}, \Delta m_{21}^{2}$ and $\Delta m_{31}^{2}$ given in table 1 , we have:

i) for $5^{\circ}<x<90^{\circ},\left(1-2 p_{1 \tau}\right)>0$ for $0 \leq \alpha_{23}<220^{\circ}$ and $500^{\circ}<\alpha_{23} \leq 720^{\circ}$, with $\left(1-2 p_{1 \tau}\right)=0$ at $\alpha_{23} \cong 220^{\circ}$ and $500^{\circ}$, where the precise values at which $\left(1-2 p_{1 \tau}\right)=0$ depend somewhat on $x$ : those corresponding to $x=10^{\circ}$ are given approximately by $\alpha_{23} \cong 250^{\circ}$ and $470^{\circ}$; for $0<x \lesssim 5^{\circ}$ we have $\left(1-2 p_{1 \tau}\right)>0$ for any $\alpha_{23}$ from the interval $\left[0,720^{\circ}\right]$;

ii) $\operatorname{sgn}\left(\Im\left(U_{\tau 2}^{*} U_{\tau 3}\right) \sin 2 x\right)>0(<0)$ for $0<\alpha_{23}<360^{\circ}\left(360^{\circ}<\alpha_{23}<720^{\circ}\right)$.

We can conclude on the basis if these observations that, except for $0<x \lesssim 5^{\circ}$, the plateau values of $\eta_{B}$

a) are negative approximately for $0<\alpha_{23}<220^{\circ}$ and $360^{\circ}<\alpha_{23}<500^{\circ}$;

b) are positive for $220^{\circ}<\alpha_{23}<360^{\circ}$ and $500^{\circ}<\alpha_{23}<720^{\circ}$.

In the case of $x$ in the interval $0<x \lesssim 5^{\circ}$, the plateau values of $\eta_{B}$ are negative (positive) for $0<\alpha_{23}<360^{\circ}\left(360^{\circ}<\alpha_{23}<720^{\circ}\right)$. However, as our numerical study shows, in this case it is possible to reproduce the observed value of $\eta_{B}$ only in a very a narrow interval of values of $x$, namely, for $2.5^{\circ}\left(1.6^{\circ}\right) \lesssim x \lesssim 5^{\circ}$ with $\alpha_{23}$ in the range $0<\alpha_{23}<250^{\circ}$ $\left(450^{\circ}<\alpha_{23}<720^{\circ}\right)$. As a consequence, this makes only a relatively small addition to regions in the space of parameters of the considered scenario for which one can have successful leptogenesis. Therefore we concentrate further on the case of $5^{\circ}<x<90^{\circ}$.

It follows from the preceding discussion that if we denote by $M_{10}^{(1,2,3,4)}$ the values of $M_{1}$ at which $\eta_{B}=0$ at the 1-to-2 flavour regime transitions taking place when $\alpha_{23}$ lies respectively in the intervals $\left(0,220^{\circ}\right),\left(360^{\circ}, 500^{\circ}\right),\left(220^{\circ}, 360^{\circ}\right)$ and $\left(500^{\circ}, 720^{\circ}\right)$, we can expect successful leptogenesis to occur for certain ranges of values of $M_{1}<M_{10}^{(1)}$ $\left(M_{1}<M_{10}^{(2)}\right)$ if $0<\alpha_{23}<220^{\circ}\left(360^{\circ}<\alpha_{23}<500^{\circ}\right)$, and of $M_{1}>M_{10}^{(3)}\left(M_{1}>M_{10}^{(4)}\right)$ extending into $\eta_{B}$ plateau region when $220^{\circ}<\alpha_{23}<360^{\circ}\left(500^{\circ}<\alpha_{23}<720^{\circ}\right)$. Moreover, the results for $90^{\circ}<x<180^{\circ}$ can be obtained from those derived for $0<x<90^{\circ}$ by making the simultaneous change $x \rightarrow \pi-x$ and $\alpha_{23} \rightarrow \alpha_{23} \pm 2 \pi$ and taking into account that the results are invariant with respect to the change $\alpha_{23} \rightarrow \alpha_{23} \pm 4 \pi$. 


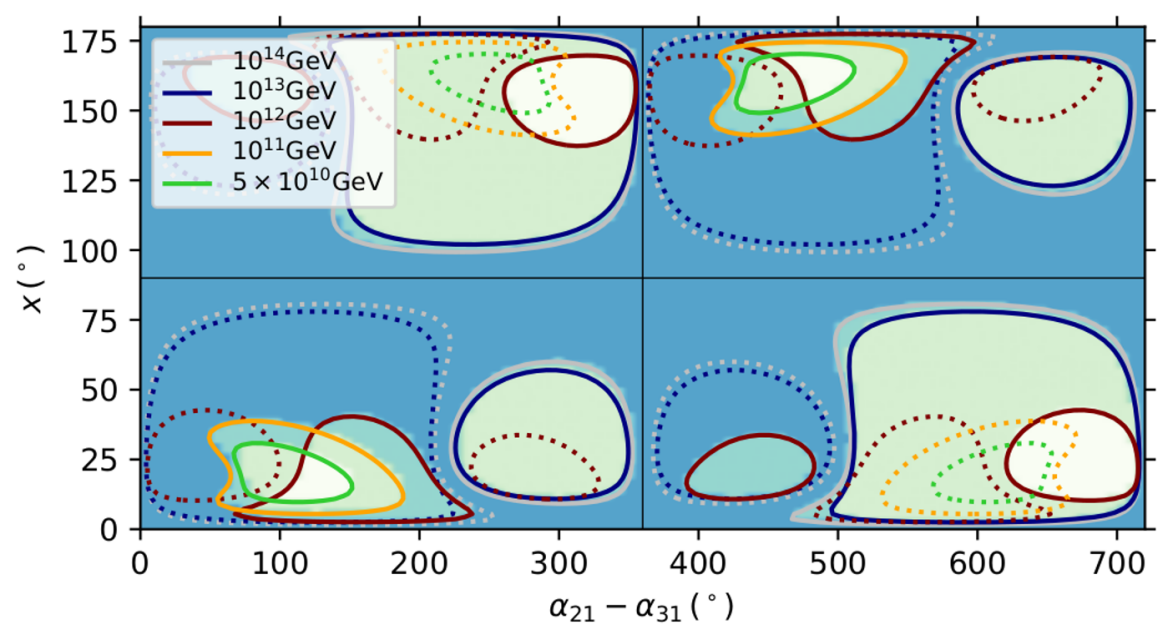

Figure 14. Regions of viable leptogenesis in the $\alpha_{23}-x$ plane for NH spectrum, $y=0, \delta=\pi$, corresponding to CP violation due to the Majorana phase $\alpha_{23}$, and different $M_{1}$. The solid contours corresponding to fixed values of $M_{1}$ surround the regions in which there is a combination of values of $\alpha_{23}$ and $x$ for which $\eta_{B}=6.1 \times 10^{-10}$. The dotted contours surround regions where one can have $\left|\eta_{B}\right|=6.1 \times 10^{-10}$ but $\eta_{B}<0$. The predicted $\eta_{B}$ outside the contours is smaller in magnitude than the present BAU. See text for further details.

These qualitative conclusions are essentially confirmed by a thorough numerical analysis, the results of which are shown graphically in figure 14. We next summarise briefly these results giving the ranges of $\alpha_{23}$ and $M_{1}$ of viable leptogenesis in the four intervals of values of $\alpha_{23}$ identified earlier for $5^{\circ}<x<90^{\circ}$. We will do it for the representative value of $x=20.5^{\circ}$ at which some of the ranges of interest are maximal, commenting first the results for the specific values of $\alpha_{23}$ at which viable leptogenesis occurs for the minimal for the case value of $M_{1}$.

- Region I: $\mathbf{0}<\boldsymbol{\alpha}_{\mathbf{2 3}}<\mathbf{2 2 0}^{\circ}$. The minimal mass scale $M_{1 \text { min }}$ for successful leptogenesis is found at $\alpha_{23}=102^{\circ}: M_{1 \min }=3.3 \times 10^{10} \mathrm{GeV}$. For $\alpha_{23}=102^{\circ}$ :

i) viable leptogenesis is possible for $3.3 \times 10^{10} \mathrm{GeV} \lesssim M_{1} \lesssim 7.7 \times 10^{11} \mathrm{GeV}$;

ii) the maximal value of the baryon asymmetry $\eta_{B}$ is reached at $M_{1} \simeq 2.55 \times$ $10^{11} \mathrm{GeV}$ and reads $\eta_{B}^{\max } \simeq 2.2 \times 10^{-9}$.

The range of $M_{1}$ and the maximal value of $\eta_{B}$ change when $\alpha_{23}$ increases (decreases) from $102^{\circ}$ to $208^{\circ}\left(64.5^{\circ}\right), 208^{\circ}\left(64.5^{\circ}\right)$ being the maximal (minimal) value of $\alpha_{23}$ at which it is possible to have successful leptogenesis (for $x=20.5^{\circ}$ ). As $\alpha_{23}$ increases to $\alpha_{23}=170^{\circ}$, the range of $M_{1}$ corresponding to $\alpha_{23}$ essentially shifts to larger values, with $\eta_{B}^{\max }$ remaining practically unchanged. More specifically, at $\alpha_{23}=120^{\circ}$, for example, the range of $M_{1}$ changes to $\left(3.3 \times 10^{10}-1.1 \times 10^{12}\right) \mathrm{GeV}$; at $170^{\circ}$ it reads $\left(8.2 \times 10^{10}-1.9 \times 10^{12}\right) \mathrm{GeV}$. At $\alpha_{23}=120^{\circ}\left(170^{\circ}\right)$, we have $\eta_{B}^{\max } \simeq 2.75(2.32) \times 10^{-9}$, which occurs at $M_{1} \simeq 3.6(6.3) \times 10^{11} \mathrm{GeV}$. 
As $\alpha_{23}$ increases further, $\eta_{B}^{\max }$ and the range of $M_{1}$ begin to decrease. At $\alpha_{23}=195^{\circ}$ we find $\eta_{B}^{\max } \simeq 1.2 \times 10^{-9}$ and $1.7 \times 10^{11} \lesssim M_{1} / \mathrm{GeV} \lesssim 1.7 \times 10^{12}$, with $\eta_{B}^{\max }$ taking place at $M_{1} \simeq 6.3 \times 10^{11} \mathrm{GeV}$. Finally, at $\alpha_{23} \simeq 208^{\circ}, \eta_{B}^{\max }$ coincides with the observed value of $\mathrm{BAU}$ and the related range of $M_{1}$ reduced to the point $M_{1} \simeq 6.3 \times 10^{11}$.

We find a similar pattern when $\alpha_{23}$ decreases from $102^{\circ}$ to $64.5^{\circ}$. At $\alpha_{23}=90^{\circ}\left(75^{\circ}\right)$, for example, we find for the range of interest of $M_{1}: 3.4(4.4) \times 10^{10} \lesssim M_{1} / \mathrm{GeV} \lesssim$ $5.2(2.7) \times 10^{11}$. The maximal asymmetry is $\eta_{B}^{\max } \simeq 1.7(1.0) \times 10^{-9}$ and occurs at $M_{1} \simeq 2.1(1.4) \times 10^{11} \mathrm{GeV}$. When $\alpha_{23}$ decreases further, the range of $M_{1}$ and $\eta_{B}^{\max }$ also decrease further and, e.g., at $\alpha_{23}=65^{\circ}$ we have: $7.8 \times 10^{10} \lesssim M_{1} / \mathrm{GeV} \lesssim 1.2 \times 10^{11}$, $\eta_{B}^{\max } \simeq 6.3 \times 10^{-10}$ which occurs at $M_{1} \simeq 9.8 \times 10^{10} \mathrm{GeV}$. At $64.5^{\circ} \eta_{B}^{\max }$ coincides with observed value of $\eta_{B}$ and the range of $M_{1}$ reduces to the point $M_{1} \simeq 9.0 \times 10^{10} \mathrm{GeV}$.

The interval of values of $\alpha_{23}$ and the related interval of values of $M_{1}$, where we can have successful leptogenesis, depend also on $x$, although this dependence is relatively weak. We find that at $x=7.5^{\circ}$ we get the largest maximal (smallest minimal) value $\alpha_{23}$ at which we still have successful leptogenesis in the considered range of $x$. These values read: $\alpha_{23}^{\min }=37^{\circ}, \alpha_{23}^{\max }=233^{\circ}$. The ranges of $M_{1}$ corresponding to $\alpha_{23}^{\min }$ and $\alpha_{23}^{\max }$ are just the points $M_{1} \simeq 2.98 \times 10^{11} \mathrm{GeV}$ and $M_{1} \simeq 6.3 \times 10^{11} \mathrm{GeV}$, respectively. For $\alpha_{23}=102^{\circ}$, for example, we have at $x=7.5^{\circ}$ : i) the range of $M_{1}$ of successful leptogenesis is $6.8 \times 10^{10} \lesssim M_{1} / \mathrm{GeV} \lesssim 1.4 \times 10^{12}$, ii) $\eta_{B}^{\max } \simeq 1.69 \times 10^{-9}$, takes place at $M_{1} \simeq 4.33 \times 10^{11} \mathrm{GeV}$ and is bigger than the observed $\eta_{B}$ by the factor $C_{M 1}=2.77$. These values should be compared with those given above for $x=20.5^{\circ}$. When $\alpha_{23}$ increases (decreases) to $233^{\circ}\left(37^{\circ}\right)$, we find quite similar behaviour of the correlation between the values of $\alpha_{23}$ and $M_{1}$ to that described for $x=20.5^{\circ}$, so we are not going to comment on it further.

- Region II: $\mathbf{2 2 0}^{\circ}<\boldsymbol{\alpha}_{\mathbf{2 3}}<\mathbf{3 6 0}^{\circ}$. The minimal mass scale $M_{1 \min }$ for successful leptogenesis is found at $\alpha_{23}=301^{\circ}: M_{1 \min }=3.1 \times 10^{12} \mathrm{GeV}$. For $\alpha_{23}=301^{\circ}$ :

i) the plateau of $\eta_{B}$ begins at $M_{1 P} \simeq 8.9 \times 10^{13} \mathrm{GeV}$;

ii) the asymmetry at the plateau $\eta_{B} \simeq 3.38 \times 10^{-9}$.

The asymmetry at the plateau is larger than the observed value of $\eta_{B}$ by the factor $C_{M 2} \simeq 5.54$. Knowing this factor allows us to determine the minimal and maximal values of $\alpha_{23}$ for having successful leptogenesis. The total range of values of $\alpha_{23}$ of interest reads:

$$
227^{\circ} \lesssim \alpha_{23} \lesssim 352^{\circ}
$$

For the corresponding range of $M_{1}$ we get: $3.11 \times 10^{12} \lesssim M_{1} / \mathrm{GeV} \lesssim 10^{14}$. with the minimal value obtained for $\alpha_{23} \simeq 301^{\circ}$.

- Region III: $360^{\circ}<\boldsymbol{\alpha}_{\mathbf{2 3}}<\mathbf{5 0 0}^{\circ}$. The minimal mass scale of leptogenesis is obtained for $\alpha_{23}=433^{\circ}$. For this choice of $\alpha_{23}$ : 

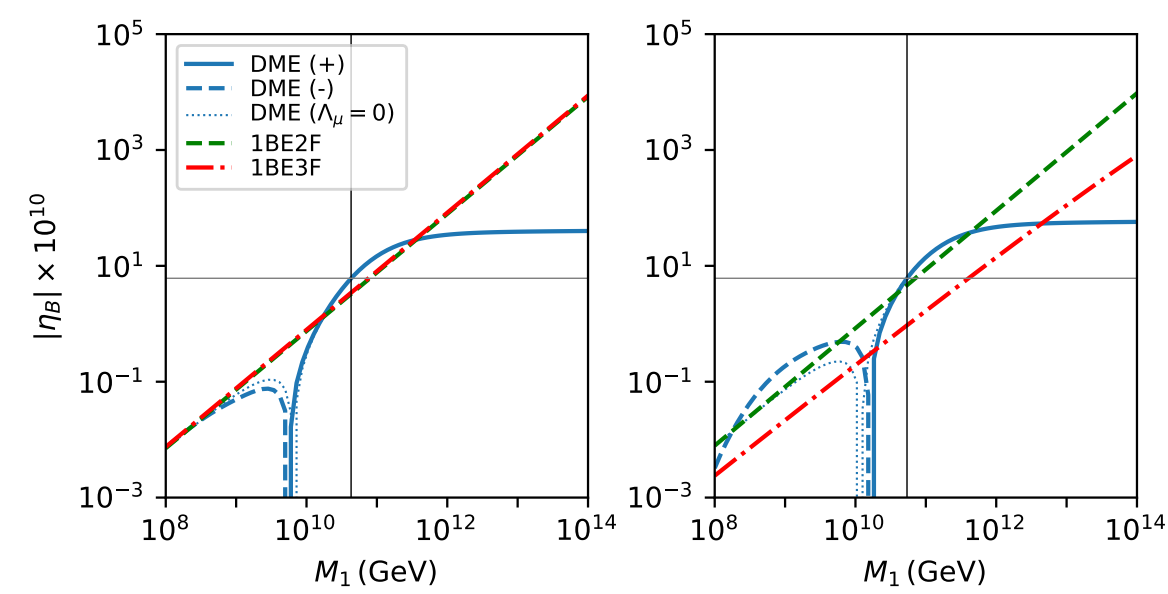

Figure 15. The asymmetry $\eta_{B}$ versus $M_{1}$ in the case of IH spectrum with CP violation due to the Majorana phase $\alpha_{21}$ (Majorana and Dirac phases $\alpha_{21}$ and $\delta$ ) of the PMNS matrix. The results shown in the left (right) panel are obtained for $x=k \pi, k=0,1,2, y \neq 0$ (purely imaginary $R_{11} R_{12}$ ) and $\alpha_{21}=164^{\circ}\left(190^{\circ}\right) \delta=\pi(3 \pi / 2)$ and $y=-76^{\circ}\left(-50^{\circ}\right)$. See text for further details.

i) leptogenesis is successful for $1.54 \times 10^{11} \lesssim M_{1} / \mathrm{GeV} \lesssim 1.59 \times 10^{12}$, where the maximal $M_{1}$ corresponds also to $\alpha_{23}=433^{\circ}$;

ii) the maximal value of $\eta_{B}$ is reached at $M_{1} \simeq 6.28 \times 10^{11} \mathrm{GeV}$ and reads $\eta_{B}^{\max } \simeq$ $1.37 \times 10^{-9}$.

The asymmetry $\eta_{B}$ at its maximum is greater than the observed value by a factor $C_{M 3} \simeq 2.25$. The range of $\alpha_{23}$ of viable leptogenesis is

$$
382^{\circ} \lesssim \alpha_{23} \lesssim 482^{\circ}
$$

- Region IV: $\mathbf{5 0 0}^{\circ}<\boldsymbol{\alpha}_{\mathbf{2 3}}<\mathbf{7 2 0 ^ { \circ }}$. The minimal $M_{1}$ for having successful leptogenesis is found at $\alpha_{23}=691^{\circ}$ and reads $M_{1} \simeq 1.53 \times 10^{11} \mathrm{GeV}$. At this value of $\alpha_{23}$,

i) the plateau of $\eta_{B}$ begins at $M_{1} \simeq 4.98 \times 10^{12} \mathrm{GeV}$;

ii) the asymmetry at the plateau $\eta_{B} \simeq 4.11 \times 10^{-9}$.

This value is larger than the observed value of $\eta_{B}$ by the factor $C_{M 4} \simeq 6.73$. Successful leptogenesis is possible for the following range of values of $\alpha_{23}$ :

$$
506^{\circ} \lesssim \alpha_{23} \lesssim 716^{\circ}
$$

For the quoted range of $\alpha_{23}$ we have viable leptogenesis for $1.54 \times 10^{11} \lesssim M_{1} / \mathrm{GeV} \lesssim$ $10^{14}$. If $x \lesssim 5^{\circ}$, the lower bound in the interval (4.26) is somewhat smaller at $\approx 470^{\circ}$, but successful leptogenesis is possible only for $M_{1} \gtrsim 10^{13} \mathrm{GeV}$.

In all four cases we have discussed there exists a correlation between the value of the CPV phase $\alpha_{23}$ and the scale of leptogenesis $M_{1}$. Thus, obtaining constraints on $\alpha_{23}$ in low-energy experiments, in principle, can constrain the leptogenesis scale of the considered scenario, or even rule out this scenario. 


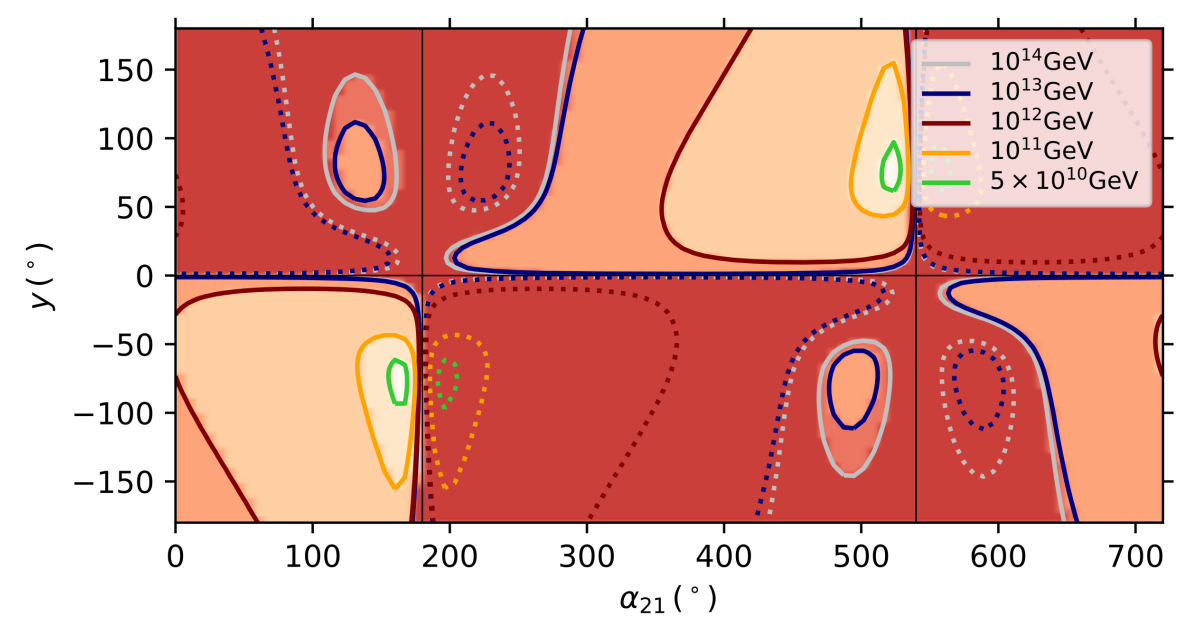

Figure 16. The regions of successful leptogenesis in the $\alpha_{21}-y$ plane in the case of IH spectrum and for $\delta=\pi$, purely imaginary $R_{11} R_{12}$ (i.e. $x=k \pi, k=0,1,2, y \neq$ ) and different $M_{1}$. The solid contours corresponding to fixed values of $M_{1}$ surround the regions where there exists a combination of values of $\alpha_{21}$ and $y$ for which $\eta_{B}=6.1 \times 10^{-10}$. The dotted contours surround regions where one can have $\left|\eta_{B}\right|=6.1 \times 10^{-10}$ but $\eta_{B}<0$. The predicted $\eta_{B}$ outside the contours is smaller in magnitude than the present BAU. The results for $0 \leq y \leq 180^{\circ}$ can be obtained from those derived for $-180^{\circ} \leq y \leq 0$ by making the simultaneous change $y \rightarrow-y$ and $\alpha_{21} \rightarrow \alpha_{21} \pm 2 \pi$. See text for further details.

IH spectrum. Viable leptogenesis in the scenario of interest is possible only for purely imaginary product $R_{11} R_{12}$ of elements of the $R$-matrix $(x=k \pi, k=0,1,2, y \neq 0)$. In figure 15 we show the modulus of $\eta_{B}$ versus $M_{1}$ for $\delta=\pi(3 \pi / 2)$ and values of the other parameters for which successful leptogenesis takes place for the minimal for the considered scenario value of $M_{1}: \alpha_{21}=164^{\circ}\left(190^{\circ}\right), y=-76^{\circ}\left(-50^{\circ}\right)$, for the left (right) panel. The right panel is obtained for $\delta=3 \pi / 2$, so it illustrates the case of viable leptogenesis with CP violation generated by both the Majorana and Dirac CPV phases of the PMNS matrix. It also illustrates the non-negligible effects the $\mathrm{CP}$ violating Dirac phase $\delta$ can have in leptogenesis when the $\mathrm{CP}$ violation is provided by the Majorana phase(s).

In both cases illustrated in figure $15, \eta_{B}$ exhibits a "non-standard" behaviour as a function of $M_{1}$ going through zero in the 1-to-2 flavour transition at $M_{1} \sim 10^{10} \mathrm{GeV} \ll$ $10^{12} \mathrm{GeV}$. In the left (right) panel the minimal value of $M_{1}$ at which the calculated $\eta_{B}$ matches the observed value of $\eta_{B}$ is $M_{1}=4.3 \times 10^{10}\left(5.4 \times 10^{10}\right) \mathrm{GeV}$. The plateau value of $\eta_{B} \cong 3.9 \times 10^{-9}\left(5.6 \times 10^{-9}\right)$ and is reached at $M_{1} \cong 1.1 \times 10^{12}\left(1.8 \times 10^{12}\right) \mathrm{GeV}$. Performing a detailed numerical analysis we have determined the regions of viable leptogenesis in the space of parameters in the considered scenario with IH spectrum, CP violation provided by $\alpha_{21}, x=k \pi, k=0,1,2, y \neq 0$ (purely imaginary $R_{11} R_{12}$ ) and $\delta=\pi$. The values of $y$ were varied in the interval $\left[-180^{\circ},+180^{\circ}\right]$. For $y=0$ we have, obviously, $\eta_{B}=0$. Due to symmetries of the quantities involved in the generation of $\eta_{B}$, the results for $0 \leq y \leq 180^{\circ}$ can formally be obtained from those derived for $-180^{\circ} \leq y \leq 0$ by making the change $y \rightarrow-y$ and $\alpha_{21} \rightarrow \alpha_{21} \pm 2 \pi$ and using the invariance with respect to $\alpha_{21} \rightarrow \alpha_{21} \pm 4 \pi$. The results of this analysis are shown graphically in figure 16. A few comments are in order. 
As we have already pointed out, the minimal value of $M_{1}$ for successful leptogenesis is found to take place at $y=-76^{\circ}$ and $\alpha_{21}=164^{\circ}$. For $y=-76^{\circ}$ and $\alpha_{21}=164^{\circ}$, the plateau value of $\eta_{B}$ given earlier is larger than the observed value of $\eta_{B}$ by the factor $C_{M 5} \simeq 6.39$. We can determine then the range of $\alpha_{21}$ for viable leptogenesis the case of $y=-76^{\circ}$ and $\delta=\pi$ from the condition:

$$
\cos \left(\alpha_{21} / 2\right)\left(1-2 p_{1 \tau}\left(\alpha_{21}\right)\right) \simeq C_{M 5}^{-1} \cos \left(82^{\circ}\right)\left(1-2 p_{1 \tau}\left(164^{\circ}\right)\right)
$$

For the range of interest we get:

$$
0 \leq \alpha_{21} \lesssim 177.5^{\circ} \text { and } 632^{\circ} \lesssim \alpha_{21} \leq 720^{\circ}
$$

When $y$ increases from $\left(-76^{\circ}\right)$ to near zero, the lower bound of the right interval decreases to $\simeq 560^{\circ}$. The corresponding range of values of $M_{1}$ extends from $M_{1} \simeq 4.3 \times 10^{10} \mathrm{GeV}$ to the beginning of the plateau at $M_{1} \cong 1.1 \times 10^{12}$ and further on the plateau at least to $M_{1} \simeq 10^{14} \mathrm{GeV}$. When $M_{1}$ decreases starting from $M_{1} \simeq 1.1 \times 10^{12}$ at the plateau to $M_{1} \simeq 4.3 \times 10^{10} \mathrm{GeV}$, the intervals of values of $\alpha_{21}$ of successful leptogenesis also decrease and at $M_{1} \simeq 4.3 \times 10^{10} \mathrm{GeV}$ shrink to the point $\alpha_{21} \simeq 164^{\circ}$.

There is an additional relatively small region of values of $\alpha_{21}$ and $M_{1}$ around $\alpha_{21} \sim$ $500^{\circ}$, for which it is possible to reproduce the observed value of BAU. It is clearly seen in figure 16. In this case the minimal mass scale takes place at $\alpha_{21} \simeq 493^{\circ}, y \simeq-76^{\circ}$ and reads $M_{1} \simeq 5.9 \times 10^{12} \mathrm{GeV}$. At the plateau we have $\eta_{B} \simeq C_{M 6} 6.1 \times 10^{-10}$, with $C_{M 6} \simeq 1.83$. The range of $\alpha_{21}$ of viable leptogenesis can be determined using the equation:

$$
\cos \left(\alpha_{21} / 2\right)\left(1-2 p_{1 \tau}\left(\alpha_{21}\right)\right) \simeq C_{M 6}^{-1} \cos \left(246.5^{\circ}\right)\left(1-2 p_{1 \tau}\left(493^{\circ}\right)\right)
$$

Solving the preceding equation we get for the range of $\alpha_{21}$ :

$$
468^{\circ} \lesssim \alpha_{21} \lesssim 522^{\circ}
$$

The corresponding range of the leptogenesis scale is $5.9 \times 10^{12} \lesssim M_{1} / \mathrm{GeV} \lesssim 10^{14}$.

As in the previous scenarios discussed by us, also in the scenario considered in the present subsection there is a correlation between the low-energy phase - in this case $\alpha_{21}$ - responsible for the $\mathrm{CP}$ violation in leptogenesis and the scale of leptogenesis $M_{1}$. Clearly, obtaining constraints on $\alpha_{21}$ in low-energy experiments can rule out the considered scenario or constrain the leptogenesis scale of the scenario.

\section{Summary and conclusions}

We have considered the generation of the baryon asymmetry of the Universe $\eta_{B}$ in the high (GUT) scale leptogenesis based on type I seesaw mechanism of neutrino mass generation. Using the density matrix equations (DME) for high scale leptogenesis in which the CP violation is provided by the low-energy Dirac or/and Majorana phases of the neutrino mixing (PMNS) matrix, we have investigated the 1-to-2 and the 2-to-3 flavour regime transitions, where the 1, 2 and 3 flavour regimes are described by the Boltzmann equations. Concentrating on the 1-to-2 flavour transitions in leptogenesis with three heavy Majorana 
neutrinos $N_{1,2,3}$ with hierarchical mass spectrum, $M_{1} \ll M_{2} \ll M_{3}$, we have determined the general conditions under which the baryon asymmetry $\eta_{B}$ goes through zero and changes sign in the transition. We have shown, in particular, that the asymmetry $\eta_{B}$ goes through zero changing its sign when leptogenesis proceeds in the strong wash-out regime with zero initial abundance of the heavy Majorana neutrinos.

In order to make the discussion of all the salient features of the transitions between the different flavour regimes of interest as transparent as possible, we have investigated further the case of decoupled heaviest Majorana neutrinos $N_{3}$, in which the number of parameters in leptogenesis is significantly smaller than in the general case of three heavy Majorana neutrinos. In particular, the complex orthogonal matrix $R$, which makes part of the Casas-Ibarra parametrisation of the neutrino Yukawa couplings we have employed in the analysis, depends only on one complex angle $\theta_{\mathrm{CI}}=x+i y$, where $x$ and $y$ are real parameters. With only two heavy Majorana neutrinos $\left(N_{1,2}\right)$ active in the seesaw mechanism, the light neutrino mass spectrum can only be either normal hierarchical (NH) with $m_{1} \cong 0$, or inverted hierarchical (IH), with $m_{3} \cong 0$. Furthermore, in the case of interest of $\mathrm{CP}$ violation in leptogenesis provided only by the low-energy CPV phases of the PMNS matrix, one can avoid the contributions to the $\mathrm{CP}$ violation associated with the $R$-matrix only if the angle $\theta_{\mathrm{CI}}$ is such that for $\mathrm{NH}(\mathrm{IH})$ spectrum $\sin 2 \theta_{\mathrm{CI}} \neq 0$ is real (purely imaginary) [18], $\sin 2 \theta_{\mathrm{CI}}=\sin 2 x\left(\sin 2 \theta_{\mathrm{CI}}= \pm i \sinh 2 y\right)$.

Analysing in detail the behaviour of $\eta_{B}$ in the transition in the case of two heavy Majorana neutrinos $N_{1,2}$ with hierarchical masses, $M_{1} \ll M_{2}$, allowed us to gain a better understanding of the transitions but also to discover new unexpected features of the transitions. We have found, in particular, that:

i) the Boltzmann equations in many cases fail to describe correctly the generation of the baryon asymmetry $\eta_{B}$ in the 1, 2 and 3 flavour regimes, in particular, underestimating $\eta_{B}$ by a factor $\sim 10$ in certain cases;

ii) depending on the values of the relevant parameters, the transitions between the different flavour regimes can be "non-standard" as, e.g., the 1-to-2 and the 2-to-3 flavour transitions can take place at the same $M_{1}$, with $\eta_{B}$ going through a relatively shallow minimum at the transition value of $M_{1}$;

iii) the two-flavour regime can persist above $5 \times 10^{11}-10^{12} \mathrm{GeV}$ (below $\sim 10^{9} \mathrm{GeV}$ ), and

iv) the flavour effects in leptogenesis persist beyond what is usually thought to be the maximum leptogenesis scale for these effects of $\sim 10^{12} \mathrm{GeV}$, with the requisite $\mathrm{CP}$ violation provided by the Dirac or/and Majorana phases present in the low-energy PMNS neutrino mixing matrix.

At $M_{1} \sim 10^{12} \mathrm{GeV},\left|\eta_{B}\right|$ reaches a "plateau" where it remains practically constant as $M_{1}$ increases and flavour effects are fully operative. We further have determined the minimal scale $M_{1 \text { min }}$ at which we can have successful leptogenesis when the CP violation is provided only by the Dirac $(\delta)$ or Majorana $\left(\alpha_{23} \equiv \alpha_{21}-\alpha_{31}\right.$ or $\left.\alpha_{21}\right)$ phases of the 
PMNS matrix as well as the ranges of the scales and the values of the phases for having successful leptogenesis. In the case of Dirac phase $\mathrm{CP}$ violation we found that $M_{1 \text { min }} \cong$ $2.5(1.7) \times 10^{11} \mathrm{GeV}$ for $\mathrm{NH}$ light neutrino mass spectrum and $\delta$ lying in the interval $\pi<\delta<2 \pi(0<\delta<\pi)$ for real (purely imaginary) Casas-Ibarra parameter $\sin 2 \theta_{\mathrm{CI}}$ (figures 7 and 9). As $M_{1}$ increases from $M_{1 \text { min }}$ to $M_{1} \cong 2.7(2.1) \times 10^{12} \mathrm{GeV}$, at which $\eta_{B}$ reaches the plateau value, the range of interest of $\delta$ increases from the point $\delta=$ $3 \pi / 2(\pi / 2)$ to $202.4^{\circ} \lesssim \delta \lesssim 337.6^{\circ}\left(14.6^{\circ} \lesssim \delta \lesssim 166.4^{\circ}\right)$ and remains practically the same up to $M_{1} \sim 10^{14} \mathrm{GeV}$. We get similar results for $M_{1 \text { min }}$ and the ranges of $\delta$ and $M_{1}$ in the case of IH light neutrino mass spectrum and purely imaginary Casas-Ibarra parameter $\sin 2 \theta_{\mathrm{CI}}$, with $M_{1 \mathrm{~min}} \cong 4.6 \times 10^{10} \mathrm{GeV}$ for $\delta=3 \pi / 2$ and $185^{\circ} \lesssim \delta \lesssim 355^{\circ}$ for $1.2 \times 10^{12} \mathrm{GeV} \lesssim M_{1} \lesssim 10^{14} \mathrm{GeV}$ (figure 12 ). We found also that in the case of $\mathrm{NH}$ spectrum there is a direct relation between the sign of $\sin \delta$ and the sign of the baryon asymmetry of the Universe in the regions of viable leptogenesis; for IH spectrum such a relation holds for $M_{1 \mathrm{~min}} \cong 4.6 \times 10^{10} \mathrm{GeV} \lesssim M_{1} \lesssim 10^{13} \mathrm{GeV}$.

We have investigated also the generation of $\eta_{B}$ when the $\mathrm{CP}$ violation is provided solely by the Majorana phases $\alpha_{21} / 2$ and $\alpha_{31} / 2$ of the PMNS matrix. In the considered scenario with two heavy Majorana neutrinos only the phase $\alpha_{23} \equiv \alpha_{21}-\alpha_{31}\left(\alpha_{21}\right)$ is physically relevant for $\mathrm{NH}(\mathrm{IH})$ light neutrino mass spectrum. We have performed a thorough analysis and have determined the ranges of values of the Majorana phase $\alpha_{23}\left(\alpha_{21}\right)$ and the related ranges of the scale $M_{1}$, for which we can successful leptogenesis with CP violation provided exclusively by $\alpha_{23}\left(\alpha_{21}\right)$ in the case of $\mathrm{NH}(\mathrm{IH})$ spectrum, real (purely imaginary) CasasIbarra factor $\sin 2 \theta_{\mathrm{CI}}=\sin 2 x \neq 0\left(\sin 2 \theta_{\mathrm{CI}}= \pm i \sinh 2 y \neq 0\right)$ and $\mathrm{CP}$ conserving value of $\delta=\pi$ (the results of these analyses are presented graphically in figures 13, 14, 15 and 16). Our results show, in particular, that there exist relatively large regions of the relevant spaces of parameters where it is possible to reproduce the observed value of BAU as well as that in these regions the values of the respective Majorana phases providing the requisite leptogenesis CP violation are strongly correlated with the value of leptogenesis scale $M_{1}$.

We note that in [71], the RGE-corrections to the neutrino Yukawa matrices are included in the calculations of the CPV lepton asymmetry through a modified Casas-Ibarra parametrisation. Without these corrections, within the formalism employed in [71], which is based on the Boltzmann equations, all lepton flavour dependence (contained in the PMNS matrix) cancels at leptogenesis scales $M_{\mathrm{LG}} \gtrsim 10^{12} \mathrm{GeV}$ from the total CPV asymmetries. Once the RGE-corrections are included, this cancellation no longer occurs and a corrective term approximately proportional to the square of the $\tau$-Yukawa coupling is added to the total CPV asymmetry. The authors of [71] then show that this correction is sufficient in some regions of the parameter space at $M_{\mathrm{LG}} \gtrsim 10^{12} \mathrm{GeV}$ for successful leptogenesis from purely low-scale CP violation due to the phases in the PMNS matrix in the absence of the usual flavour effects. However, this correction is subdominant to flavour effects discussed in this work, typically being by a factor of $\sim 10$ to $\sim 100$ smaller in the regions of the parameter space of the scenarios we have considered, where leptogenesis successfully generates the observed BAU. Thus, the mechanism of generation of BAU considered in [71] is subdominant to the mechanism discussed in this work. 
It follows from the results obtained in the present article that, in particular, viable leptogenesis based on type I seesaw mechanism with two hierarchical in mass heavy Majorana neutrinos and CP violation provided by the physical low-energy Dirac or/and Majorana phases, present in the PMNS neutrino mixing matrix, is possible for rather wide ranges of values of the $\mathrm{CP}$ violating phases and of the scale of leptogenesis. The scenarios of leptogenesis investigated by us are obviously falsifiable in low-energy experiments on the nature - Dirac or Majorana - of massive neutrinos. As far as the nature of massive neutrinos is not known or if the massive neutrinos are proven to be Majorana particles, the cases of leptogenesis we have considered are still testable and falsifiable in low-energy experiments on $\mathrm{CP}$ violation in neutrino oscillations, on determination of the type of spectrum neutrino masses obey and of the absolute neutrino mass scale. The data from these experiments can severely constrain the corresponding leptogenesis parameter spaces and even rule out some of, if not all, the cases studied in detail by us. We are looking forward to these experimental data that can provide crucial tests of the leptogenesis scenarios discussed in the present paper.

\section{Acknowledgments}

This work was supported in part by the INFN program on Theoretical Astroparticle Physics (A.G. and S.T.P.) and by the World Premier International Research Center Initiative (WPI Initiative, MEXT), Japan (S.T.P.). K.M. acknowledges the (partial) support from the European Research Council under the European Union Seventh Framework Programme (FP/2007-2013) / ERC Grant NuMass agreement n. [617143].

\section{A DMEs from the three- to the two-flavour basis}

We consider here the scenario of two-flavoured leptogenesis within the DMEs. This is equivalent to set $\Gamma_{\mu} / H z=0$ in the DMEs defined in eqs. (2.42)-(2.43). We describe here in detail how to arrive from the DMEs in the three-flavour basis in eqs. (2.42)-(2.43) to the ones in the two-flavour basis given in eqs. (3.1)-(3.4). We also discuss how the formal solution to those is obtained and how, under the single-flavour approximation, they recover the single-flavoured Boltzmann equations in eqs. (2.24)-(2.25).

In the calculations that follow, it will prove convenient to use the CP-asymmetry in eqs. (2.46) also for the $\tau^{\perp}$-flavour and for the $i^{\text {th }}$ heavy Majorana neutrino $N_{i}$, which, in terms of the $C_{i \alpha}$ coefficients defined in eq. (2.21), is given by

$$
\begin{aligned}
\epsilon_{\alpha \beta}^{(i)}= & \frac{3}{32 \pi} \sum_{j \neq i}\left(Y^{\dagger} Y\right)_{j j}\left\{i\left[C_{i \alpha} C_{j \beta}^{*}\left(C^{\dagger} C\right)_{j i}-C_{i \beta}^{*} C_{j \alpha}\left(C^{\dagger} C\right)_{i j}\right] f_{1}\left(\frac{x_{j}}{x_{i}}\right)\right. \\
& \left.+i\left[C_{i \alpha} C_{j \beta}^{*}\left(C^{\dagger} C\right)_{i j}-C_{i \beta}^{*} C_{j \alpha}\left(C^{\dagger} C\right)_{j i}\right] f_{2}\left(\frac{x_{j}}{x_{i}}\right)\right\},
\end{aligned}
$$

with $i=1,2,3$ and $\alpha, \beta=e, \mu, \tau, \tau^{\perp}$. Explicitly, the diagonal terms are

$$
\epsilon_{\alpha \alpha}^{(i)}=\frac{3}{16 \pi} \sum_{j \neq i}\left(Y^{\dagger} Y\right)_{j j}\left\{\Im\left[C_{i \alpha}^{*} C_{j \beta}\left(C^{\dagger} C\right)_{i j}\right] f_{1}\left(\frac{x_{j}}{x_{i}}\right)+\Im\left[C_{i \alpha}^{*} C_{j \beta}\left(C^{\dagger} C\right)_{j i}\right] f_{2}\left(\frac{x_{j}}{x_{i}}\right)\right\} .
$$


We remind also that $\left(Y^{\dagger} Y\right)_{j j}=\sum_{\gamma}\left|Y_{\gamma j}\right|^{2},\left(C^{\dagger} C\right)_{i j}=\sum_{\gamma} C_{i \gamma}^{*} C_{j \gamma}$ and $p_{i \gamma} \equiv\left|C_{i \gamma}\right|^{2}$ with $\gamma=e, \mu, \tau$, while $p_{i \tau^{\perp}} \equiv p_{i e}+p_{i \mu}$ and $p_{i \tau}+p_{i \tau^{\perp}}=p_{i \tau}+p_{i e}+p_{i \mu}=1$. To use eqs. (A.1) and (A.2) we should define the coefficients $C_{i \tau^{\perp}}$ and $C_{i \tau^{\perp}}^{*}$. However, the relation $\epsilon_{\tau^{\perp} \tau^{\perp}}^{(i)} \equiv$ $\epsilon_{e e}^{(i)}+\epsilon_{\mu \mu}^{(i)}$ imposes that

$$
C_{i \tau^{\perp}} C_{j \tau^{\perp}}^{*}=C_{i e} C_{j e}^{*}+C_{i \mu} C_{j \mu}^{*},
$$

which for $i=j$ means $\left|C_{i \tau^{\perp}}\right|^{2}=\left|C_{i e}\right|^{2}+\left|C_{i \mu}\right|^{2}=p_{i \tau^{\perp}}$. Since the physical quantities (e.g., $N_{B-L}$ ) depend on $\left|C_{i \tau^{\perp}}\right|^{2}$ (in our case of interest $i=1$ ), there is actually no need to define the coefficients $C_{i \tau^{\perp}}$ and $C_{i \tau^{\perp}}^{*}$, apart from imposing the constraint in eq. (A.3), so we are going to let them free in our calculations.

An important relation that derives from eqs. (A.1) and (A.2) and that is going to be used further is

$$
2 \Re\left[C_{i \beta} C_{i \alpha}^{*} \epsilon_{\alpha \beta}^{(i)}\right]=p_{i \beta} \epsilon_{\alpha \alpha}^{(i)}+p_{i \alpha} \epsilon_{\beta \beta}^{(i)},
$$

with $\alpha, \beta=e, \mu, \tau, \tau^{\perp}$.

We now concentrate again on the hierarchical case for which only the decay of the heavy neutrino $N_{1}$ is relevant for leptogenesis (i.e. $i=1$ ). Firstly, we sum the equations for $N_{e e}$ and $N_{\mu \mu}$, which result from taking $\alpha=\beta=e, \mu$ in eqs. (2.43) respectively, and get an equation for $N_{\tau^{\perp} \tau^{\perp}}=N_{e e}+N_{\mu \mu}$ :

$$
\begin{aligned}
\frac{d N_{\tau^{\perp} \tau^{\perp}}}{d z}= & \epsilon_{\tau^{\perp} \tau^{\perp}}^{(1)} D_{1}\left(N_{N_{1}}-N_{N_{1}}^{e q}\right)+ \\
& -W_{1}\left\{p_{1 e} N_{e e}+p_{1 \mu} N_{\mu \mu}+2 \Re\left[C_{1 e} C_{1 \mu}^{*} N_{\mu e}\right]+\right. \\
& \left.+\Re\left[C_{1 e} C_{1 \tau}^{*} N_{\tau e}\right]+\Re\left[C_{1 \mu} C_{1 \tau}^{*} N_{\tau \mu}\right]\right\} .
\end{aligned}
$$

The second line of the above equation is actually $p_{1 \tau^{\perp}} N_{\tau^{\perp} \tau^{\perp}}$. This can be shown by considering the equations for $p_{1 \mu} N_{e e}, p_{1 e} N_{\mu \mu}$ and $2 \Re\left[C_{1 e} C_{1 \mu}^{*} N_{e e}\right]$, from which we can write:

$$
\begin{aligned}
2 \Re\left[C_{1 e} C_{1 \mu}^{*} \frac{d N_{\mu e}}{d z}\right]= & 2 \Re\left[C_{1 e} C_{1 \mu}^{*} \epsilon_{\mu e}^{(1)}\right] D_{1}\left(N_{N_{1}}-N_{N_{1}}^{e q}\right)+ \\
- & W_{1} \Re\left[C_{1 e} C_{1 \mu}^{*}\left\{P^{0(1)}, N\right\}_{\mu e}\right] \\
= & \left(p_{1 \mu} \epsilon_{e e}^{(1)}+p_{1 e} \epsilon_{\mu \mu}^{(1)}\right) D_{1}\left(N_{N_{1}}-N_{N_{1}}^{e q}\right)+ \\
& -W_{1}\left\{p_{1 e} p_{1 \mu}\left(N_{e e}+N_{\mu \mu}\right)+\right. \\
& \left.+\left(p_{1 e}+p_{1 \mu}\right) \Re\left[C_{1 e} C_{1 \mu}^{*} N_{\mu e}\right]+p_{1 \mu} \Re\left[C_{1 e} C_{1 \tau}^{*} N_{\tau e}\right]+p_{1 e} \Re\left[C_{1 \mu} C_{1 \tau}^{*} N_{\tau \mu}\right]\right\} \\
= & p_{1 \mu} \frac{d N_{e e}}{d z}+p_{1 e} \frac{d N_{\mu \mu}}{d z} .
\end{aligned}
$$


By assuming that at the beginning of leptogenesis $\left(z_{0}\right)$ all the asymmetries are zero, the following condition must hold at any $z \geq 0::^{23}$

$$
2 \Re\left[C_{1 e} C_{1 \mu}^{*} N_{\mu e}\right]=p_{1 \mu} N_{e e}+p_{1 e} N_{\mu \mu}
$$

which leads to

$$
p_{1 e} N_{e e}+p_{1 \mu} N_{\mu \mu}+2 \Re\left[C_{1 e} C_{1 \mu}^{*} N_{\mu e}\right]=p_{1 \tau^{\perp}} N_{\tau^{\perp} \tau^{\perp}}
$$

We then define

$$
N_{\tau \tau^{\perp}} \equiv\left(\frac{C_{1 e}}{C_{1 \tau^{\perp}}} N_{\tau e}+\frac{C_{1 \mu}}{C_{1 \tau^{\perp}}} N_{\tau \mu}\right)
$$

and $N_{\tau^{\perp} \tau}=N_{\tau \tau^{\perp}}^{*}$, so that the equations for $N_{\tau \tau}$ and $N_{\tau^{\perp} \tau^{\perp}}$ can be recast in the forms given in eqs. (3.2) and (3.3). By using the relation $C_{i \tau^{\perp}} \epsilon_{\tau \tau^{\perp}}^{(i)}=C_{i e} \epsilon_{\tau e}^{(i)}+C_{i \mu} \epsilon_{\tau \mu}^{(i)}$ (with $i=1$ in our case), which follows from eqs. (A.1) and (A.3), combined with all the previous relations, we get the equation for $N_{\tau \tau^{\perp}}$ as in eq. (3.4) and the DMEs in the two-flavour basis are recovered.

The formal expression of $N_{\tau \tau^{\perp}}$ can be obtained by solving eq. (3.4) with the integrating factor method, which leads to

$$
\begin{aligned}
N_{\tau \tau^{\perp}}(z)= & \epsilon_{\tau \tau^{\perp}}^{(1)} \int_{z_{0}}^{z} D_{1}\left(z^{\prime}\right)\left(N_{N_{1}}\left(z^{\prime}\right)-N_{N_{1}}^{e q}\left(z^{\prime}\right)\right) e^{-\Lambda_{\tau}\left(z-z^{\prime}\right)} e^{-\frac{1}{2} \int_{z^{\prime}}^{z} W_{1} d z^{\prime \prime}} d z^{\prime}+ \\
& -\frac{1}{2} C_{1 \tau} C_{1 \tau^{\perp}}^{*} \int_{z_{0}}^{z} W_{1}\left(z^{\prime}\right) N_{B-L}\left(z^{\prime}\right) e^{-\Lambda_{\tau}\left(z-z^{\prime}\right)} e^{-\frac{1}{2} \int_{z^{\prime}}^{z} W_{1}\left(z^{\prime \prime}\right) d z^{\prime \prime}} d z^{\prime}
\end{aligned}
$$

where the initial asymmetry was assumed to be zero, namely $N_{\tau \tau^{\perp}}\left(z_{0}\right)=0$. Notice that the above expression contains a term with $N_{B-L}$ which cannot be ignored in general, if not, e.g., in the limit of $\Gamma_{\tau} / H z \rightarrow 0$ (see section 3).

To get the resulting equation for $N_{B-L}=N_{\tau \tau}+N_{\tau^{\perp} \tau^{\perp}}$ we first notice that:

$$
\begin{aligned}
2 \Re\left[C_{1 \tau^{\perp}} C_{1 \tau}^{*} \frac{d N_{\tau \tau^{\perp}}}{d z}\right]= & 2 \Re\left[C_{1 \tau^{\perp}} C_{1 \tau}^{*} \epsilon_{\tau \tau^{\perp}}^{(1)}\right] D_{1}\left(N_{N_{1}}-N_{N_{1}}^{e q}\right)+ \\
& -W_{1}\left\{\Re\left[C_{1 \tau^{\perp}} C_{1 \tau^{\prime}}^{*} N_{\tau^{\perp}}\right]+p_{1 \tau} p_{1 \tau^{\perp}} N_{B-L}\right\}-2 \Re\left[C_{1 \tau^{\perp}} C_{1 \tau^{*}}^{*} N_{\tau \tau^{\perp}} \frac{\Gamma_{\tau}}{H z}\right] \\
= & \left(p_{1 \tau^{\perp}} \epsilon_{\tau \tau}^{(1)}+p_{1 \tau} \epsilon_{\tau^{\perp} \tau^{\perp}}^{(1)}\right) D_{1}\left(N_{N_{1}}-N_{N_{1}}^{e q}\right)+ \\
& -W_{1}\left\{\Re\left[C_{1 \tau^{\perp}} C_{1 \tau^{\prime}}^{*} N_{\tau \tau^{\perp}}\right]+p_{1 \tau} p_{1 \tau^{\perp}} N_{B-L}\right\}-2 \Re\left[C_{1 \tau^{\perp}} C_{1 \tau}^{*} N_{\tau \tau^{\perp}} \frac{\Gamma_{\tau}}{H z}\right]
\end{aligned}
$$

Then we write the equation for $p_{1 \tau^{\perp}} N_{\tau \tau}+p_{1 \tau} N_{\tau^{\perp} \tau^{\perp}}$, that is:

$$
\begin{aligned}
\frac{d}{d z}\left(p_{1 \tau^{\perp}} N_{\tau \tau}+p_{1 \tau} N_{\tau^{\perp} \tau^{\perp}}\right)= & \left(p_{1 \tau^{\perp}} \epsilon_{\tau \tau}^{(1)}+p_{1 \tau} \epsilon_{\tau^{\perp} \tau^{\perp}}^{(1)}\right) D_{1}\left(N_{N_{1}}-N_{N_{1}}^{e q}\right)+ \\
& -W_{1}\left\{\Re\left[C_{1 \tau^{\perp}} C_{1 \tau^{*}}^{*} N_{\tau \tau^{\perp}}\right]+p_{1 \tau} p_{1 \tau^{\perp}} N_{B-L}\right\} \\
= & 2 \Re\left[C_{1 \tau^{\perp}} C_{1 \tau}^{*} \frac{d N_{\tau \tau^{\perp}}}{d z}\right]+2 \Re\left[C_{1 \tau^{\perp}} C_{1 \tau^{*}}^{*} N_{\tau \tau^{\perp}} \frac{\Gamma_{\tau}}{H z}\right]
\end{aligned}
$$

\footnotetext{
${ }^{23}$ We stress that this is only valid if $\Gamma_{\mu} / H z=0$, as in our case.
} 
Then, given that

$$
p_{1 \tau^{\perp}} N_{\tau \tau}+p_{1 \tau} N_{\tau^{\perp} \tau^{\perp}}=N_{B-L}-\left(p_{1 \tau} N_{\tau \tau}+p_{1 \tau^{\perp}} N_{\tau^{\perp} \tau^{\perp}}\right)
$$

we get

$$
p_{1 \tau} \frac{d N_{\tau \tau}}{d z}+p_{1 \tau^{\perp}} \frac{d N_{\tau^{\perp} \tau^{\perp}}}{d z}+2 \Re\left[C_{1 \tau^{\perp}} C_{1 \tau}^{*} \frac{d N_{\tau \tau^{\perp}}}{d z}\right]=\frac{d N_{B-L}}{d z}-2 \Re\left[C_{1 \tau^{\perp}} C_{1 \tau^{*}}^{*} N_{\tau \tau^{\perp}} \frac{\Gamma_{\tau}}{H z}\right] .
$$

Since all the asymmetries are assumed to be zero at $z_{0}$, the above relation converts to

$$
p_{1 \tau} N_{\tau \tau}(z)+p_{1 \tau^{\perp}} N_{\tau^{\perp} \tau^{\perp}}(z)+2 \Re\left[C_{1 \tau^{\perp}} C_{1 \tau^{\prime}}^{*} N_{\tau \tau^{\perp}}(z)\right]=N_{B-L}(z)-\lambda(z),
$$

with $\lambda(z)$ defined as in eq. (3.8). We note that $\lambda(z)=0$ in the single-flavour approximation, namely for $\Gamma_{\tau} / H z=0$.

Finally, by summing eqs. (3.2) and (3.3) and using the previous relations we get an equation for $N_{B-L}$ that reads:

$$
\frac{d N_{B-L}}{d z}=\epsilon^{(1)} D_{1}(z)\left(N_{N_{1}}(z)-N_{N_{1}}^{e q}(z)\right)-W_{1}(z) N_{B-L}(z)+W_{1}(z) \lambda(z) .
$$

In the case of $\lambda(z)=0$, the above equation corresponds to the Boltzmann equation for the $B-L$ asymmetry in the single-flavour approximation given in eq. (2.25). Moreover, when $\epsilon^{(1)}=0$, as in the case of CP violation solely provided by the PMNS phases, eq. (A.16) reduces to eq. (3.19).

The formal solution to eq. (A.16) reads:

$$
\begin{aligned}
N_{B-L}(z)= & \int_{z_{0}}^{z} e^{-\int_{z^{\prime}}^{z} W_{1}\left(z^{\prime \prime}\right) d z^{\prime \prime}} \epsilon^{(1)} D_{1}\left(z^{\prime}\right)\left(N_{N_{1}}\left(z^{\prime}\right)-N_{N_{1}}^{e q}\left(z^{\prime}\right)\right) d z^{\prime} \\
& +\int_{z_{0}}^{z} W_{1}\left(z^{\prime}\right) \lambda\left(z^{\prime}\right) e^{-\int_{z^{\prime}}^{z} W_{1} d z^{\prime \prime}} d z^{\prime},
\end{aligned}
$$

where, as usual, we have assumed vanishing initial asymmetry $N_{B-L}\left(z_{0}\right)=0$.

\section{B Approximated solutions to the BEs in various regimes}

In this appendix we illustrate the passages that lead to the analytical approximations to the Boltzmann Equations (BEs) in various regimes. Useful references with similar calculations are $[64,72]$. The BEs are:

$$
\begin{aligned}
& \frac{d N_{N_{1}}}{d z}=-D_{1}\left(N_{N_{1}}-N_{N_{1}}^{e q}\right) \\
& \frac{d N_{\alpha \alpha}}{d z}=\epsilon_{\alpha \alpha}^{(1)} D_{1}\left(N_{N_{1}}-N_{N_{1}}^{e q}\right)-W_{1} p_{1 \alpha} N_{\alpha \alpha},
\end{aligned}
$$

where $\alpha=\tau, \tau^{\perp}$ or $e, \mu, \tau$ in the two- or three-flavour basis, respectively. The single-flavour BEs can be recovered by formally substituting $N_{\alpha \alpha}$ with $N_{B-L}$ and setting $p_{1 \alpha}=1$ in (B.2). The strength of the decays and inverse decays is quantified by $\kappa_{1} p_{1 \alpha}$. When $\kappa_{1} p_{1 \alpha} \gg 1$, the flavour $\alpha$ is said to be in the strong wash-out regime. Conversly, if $\kappa_{1} p_{1 \alpha} \ll 1$, the 
flavour $\alpha$ is in the weak wash-out regime. The formal solution to the BEs can be found by means of the integrating factor method:

$$
\begin{aligned}
N_{N_{1}}(z)= & N_{N_{1}}\left(z_{0}\right) e^{-\int_{z_{0}}^{z} D_{1}\left(z^{\prime}\right) d z^{\prime}}+\int_{z_{0}}^{z} D_{1}\left(z^{\prime}\right) N_{N_{1}}^{e q}\left(z^{\prime}\right) e^{-\int_{z^{\prime}}^{z} D_{1}\left(z^{\prime \prime}\right) d z^{\prime \prime}} d z^{\prime}, \\
N_{\alpha \alpha}(z)= & N_{\alpha \alpha}\left(z_{0}\right) e^{-\int_{z_{0}}^{z} W_{1}\left(z^{\prime}\right) p_{1 \alpha} d z^{\prime}}+ \\
& +\epsilon_{\alpha \alpha}^{(1)} \int_{z_{0}}^{z} D_{1}\left(z^{\prime}\right)\left(N_{N_{1}}\left(z^{\prime}\right)-N_{N_{1}}^{e q}\left(z^{\prime}\right)\right) e^{-\int_{z^{\prime}}^{z} W_{1}\left(z^{\prime \prime}\right) p_{1 \alpha} d z^{\prime \prime}} d z^{\prime} .
\end{aligned}
$$

Assuming zero asymmetry at $z_{0}$, the first term in eq. (B.4) vanishes.

\section{B.1 Strong wash-out regime}

In the strong wash-out regime for a certain lepton flavour $\alpha$, there is a period $z_{\alpha}^{\text {in }} \leq z \leq z_{\alpha}^{\text {out }}$ for which $W_{1}(z) p_{1 \alpha} \geq 1$. Assuming that the wash-outs are effective enough, any asymmetry in the flavour $\alpha$ generated before $z_{\alpha}^{i n}$ is fully erased by wash-outs. Therefore, there is no dependence on the initial condition in this case. An analytical approximation for the asymmetry for $z_{\alpha}^{\text {in }} \leq z \leq z_{\alpha}^{\text {out }}$ can then be found by setting the right-hand side of eq. (B.2) to zero (this corresponds to the so-called strong wash-out balance approximation [70, 72]):

$$
N_{\alpha \alpha}(z) \simeq-\frac{\epsilon_{\alpha \alpha}^{(1)}}{W_{1} p_{1 \alpha}} \frac{d N_{N_{1}}}{d z} \simeq-\frac{\epsilon_{\alpha \alpha}^{(1)}}{W_{1} p_{1 \alpha}} \frac{d N_{N_{1}}^{e q}}{d z}=\frac{2 N_{\ell}^{e q}}{z \kappa_{1} p_{1 \alpha}} \epsilon_{\alpha \alpha}^{(1)}
$$

where we have used the approximation $N_{N_{1}}(z) \simeq N_{N_{1}}^{e q}(z)=\frac{1}{2} z^{2} K_{2}(z) N_{N}^{e q}(0)$ valid in this regime and $d\left(z^{2} K_{2}(z)\right) / d z=-z^{2} K_{1}(z)$. After $z_{\alpha}^{\text {out }}$, the asymmetry in the flavour $\alpha$ gets frozen so that:

$$
N_{\alpha \alpha}(\infty) \simeq \frac{2 N_{\ell}^{e q}}{\kappa_{1}} \frac{\epsilon_{\alpha \alpha}^{(1)}}{z_{\alpha}^{\text {out }} p_{1 \alpha}},
$$

with $z_{\alpha}^{\text {out }} \simeq 1.25 \ln \left(25 \kappa_{1} p_{1 \alpha}\right)$.

In the two-flavour approximation, since usually $z_{d} \equiv z_{\tau}^{\text {out }} \simeq z_{\tau^{\perp}}^{\text {out }}$, the final $B-L$ asymmetry is given by

$$
N_{B-L}^{1 \mathrm{BE} 2 \mathrm{~F}}(\infty) \simeq \frac{2 N_{\ell}^{e q}}{z_{d} \kappa_{1}} \frac{\epsilon_{\tau \tau}^{(1)} p_{1 \tau^{\perp}}+\epsilon_{\tau^{\perp} \tau^{\perp}}^{(1)} p_{1 \tau}}{p_{1 \tau} p_{1 \tau^{\perp}}}
$$

\section{B.2 Weak wash-out regime}

In the weak wash-out regime we need to distinguish between two different initial conditions, namely thermal initial abundance (TIA) and vanishing initial abundance (VIA) for which $N_{N_{1}}\left(z_{0}\right)=N_{N_{1}}^{e q}\left(z_{0}\right)$ and $N_{N_{1}}\left(z_{0}\right)=0$ respectively.

\section{B.2.1 Vanishing initial abundance}

The number of heavy neutrinos evolving with $z$ in the VIA case, for which $N_{N_{1}}\left(z_{0}\right)=0$, follows from eq. (B.3):

$$
\begin{aligned}
N_{N_{1}}(z) & \simeq \int_{z_{0}}^{z} D_{1}\left(z^{\prime}\right) N_{N_{1}}^{e q}\left(z^{\prime}\right) e^{-\int_{z^{\prime}}^{z} D_{1}\left(z^{\prime \prime}\right) d z^{\prime \prime}} d z^{\prime} \\
& =2 N_{\ell}^{e q} \int_{z_{0}}^{z} W_{1}\left(z^{\prime}\right) e^{-\int_{z^{\prime}}^{z} D_{1}\left(z^{\prime \prime}\right) d z^{\prime \prime}} d z^{\prime}
\end{aligned}
$$


We define $z_{e q}$ as the time at which $N_{N_{1}}\left(z_{e q}\right)=N_{N_{1}}^{e q}\left(z_{e q}\right)$, that corresponds to a maximum for $N_{N_{1}}(z)$. Indeed, from eq. (B.1) it follows that, at $z_{e q}, d N_{N_{1}} / d z=0$ and $d^{2} N_{N_{1}} / d z^{2}=D_{1} d N_{N_{1}}^{e q} / d z<0$. The number of RH neutrinos at $z_{e q}$ can be computed using some analytical approximations such as in [64], of which we employ the same result:

$$
N\left(\kappa_{1}\right) \equiv N_{N_{1}}\left(z_{e q}\right) \simeq \frac{9 \pi}{16} \kappa_{1} .
$$

For $z<z_{e q}$, we can assume $N_{N_{1}}^{e q} \gg N_{N_{1}}$. Then, from eq. (B.4) and using eq. (2.29), we find that the asymmetry up to $z_{e q}$ reads:

$$
\begin{aligned}
N_{\alpha \alpha}\left(z_{e q}\right) & \simeq \epsilon_{\alpha \alpha}^{(1)} \int_{z_{0}}^{z_{e q}} D_{1}\left(z^{\prime}\right) N_{N_{1}}^{e q}\left(z^{\prime}\right) e^{-\int_{z^{\prime}}^{z_{e q}} W_{1}\left(z^{\prime \prime}\right) p_{1 \alpha} d z^{\prime \prime}} d z^{\prime} \\
& =2 N_{\ell}^{e q} \frac{\epsilon_{\alpha \alpha}^{(1)}}{p_{1 \alpha}}\left(1-e^{-p_{1 \alpha} \frac{N\left(\kappa_{1}\right)}{2 N_{\ell}^{e q}}}\right) \simeq-N\left(\kappa_{1}\right) \epsilon_{\alpha \alpha}^{(1)}+\frac{N\left(\kappa_{1}\right)^{2}}{4 N_{\ell}^{e q}} \epsilon_{\alpha \alpha}^{(1)} p_{1 \alpha} .
\end{aligned}
$$

For $z>z_{e q}$, we can instead write the asymmetry as:

$$
\begin{aligned}
N_{\alpha \alpha}(z)-N_{\alpha \alpha}\left(z_{e q}\right) & =-\epsilon_{\alpha \alpha}^{(1)} \int_{z_{e q}}^{z} \frac{d N_{N 1}}{d z^{\prime}} e^{-\int_{z^{\prime}}^{z} W_{1}\left(z^{\prime \prime}\right) p_{1 \alpha} d z^{\prime \prime}} d z^{\prime} \\
& \simeq-\epsilon_{\alpha \alpha}^{(1)} \int_{z_{e q}}^{z} \frac{d N_{N 1}}{d z^{\prime}}\left(1-p_{1 \alpha} \int_{z^{\prime}}^{z} W_{1}\left(z^{\prime \prime}\right) d z^{\prime \prime}\right) d z^{\prime} \\
& \simeq \epsilon_{\alpha \alpha}^{(1)}\left(N\left(\kappa_{i}\right)-N_{N_{1}}(z)\right)-p_{1 \alpha} \epsilon_{\alpha \alpha}^{(1)} \int_{z_{e q}}^{z} d z^{\prime} D_{1}\left(z^{\prime}\right) N_{N_{1}}\left(z^{\prime}\right) \int_{z^{\prime}}^{z} d z^{\prime \prime} W_{1}\left(z^{\prime \prime}\right) \\
& \simeq \epsilon_{\alpha \alpha}^{(1)}\left(N\left(\kappa_{i}\right)-N_{N_{1}}(z)\right),
\end{aligned}
$$

where in the last passage we have neglected the (negative) term proportional to $p_{1 \alpha}$. This last approximation may be a bit inaccurate if $10^{-2}<\kappa_{1} p_{1 \alpha}<1$ [64].

The final asymmetry then reads $\left(N_{N_{1}}(\infty)=0\right)$ :

$$
N_{\alpha \alpha}(\infty) \simeq \epsilon_{\alpha \alpha}^{(1)} p_{1 \alpha} \frac{N\left(\kappa_{1}\right)^{2}}{4 N_{\ell}^{e q}} \simeq \frac{81 \pi^{2}}{1024 N_{\ell}^{e q}} \kappa_{1}^{2} \epsilon_{\alpha \alpha}^{(1)} p_{1 \alpha},
$$

which, in the two-flavour approximations results in

$$
N_{B-L}^{1 \mathrm{BE} 2 \mathrm{~F}}(\infty) \simeq \frac{81 \pi^{2}}{1024 N_{\ell}^{e q}} \kappa_{1}^{2}\left(\epsilon_{\tau \tau}^{(1)} p_{1 \tau}+\epsilon_{\tau^{\perp} \tau^{\perp}}^{(1)} p_{1 \tau^{\perp}}\right)
$$

\section{B.2.2 Thermal initial abundance}

In the TIA case, $N_{N_{1}}\left(z_{0}\right)=N_{N_{1}}^{e q}\left(z_{0}\right)$. We define $z_{D}$ so that $z_{D} D_{1}\left(z_{D}\right)=2$, i.e. as the time at which decays are in equilibrium against the expanding Universe. In the weak wash-out regime $z_{D} \gg 1$.

For $z \lesssim 1$ we can consider $N_{N_{1}}^{e q}(z) \simeq N_{N_{1}}^{e q}\left(z_{0}\right)$. Hence,

$$
\begin{aligned}
N_{N_{1}}(z) & \simeq N_{N_{1}}^{e q}\left(z_{0}\right) \int_{z_{0}}^{z} D_{1} e^{-\int_{z^{\prime}}^{z} D_{1}\left(z^{\prime \prime}\right) d z^{\prime \prime}} d z^{\prime}+N_{N_{1}}^{e q}\left(z_{0}\right) e^{-\int_{z_{0}}^{z} D_{1}\left(z^{\prime}\right) d z^{\prime}} \\
& =N_{N_{1}}^{e q}\left(z_{0}\right)\left(1-e^{-\int_{z_{0}}^{z} D_{1}\left(z^{\prime}\right) d z^{\prime}}\right)+N_{N_{1}}^{e q}\left(z_{0}\right) e^{-\int_{z_{0}}^{z} D_{1}\left(z^{\prime}\right) d z^{\prime}}=N_{N_{1}}^{e q}\left(z_{0}\right) .
\end{aligned}
$$


For $1<z \leq z_{D}$, the equilibrium number density is exponentially dropped so that $N_{N_{1}}^{e q}(z) \ll$ $N_{N_{1}}(z)$ and we have

$$
N_{N_{1}}(z) \simeq N_{N_{1}}^{e q}\left(z_{0}\right) e^{-\int_{1}^{z} D_{1}\left(z^{\prime}\right) d z^{\prime}} \simeq N_{N_{1}}^{e q}\left(z_{0}\right)
$$

Then the asymmetry up to $z_{D}$ is roughly zero. At $z \simeq z_{D}$ the heavy neutrinos start to decay effectively and their abundance for $z \gtrsim z_{D}$ is exponentially damped:

$$
N_{N_{1}}(z) \simeq N_{N_{1}}^{e q}\left(z_{0}\right) e^{-\int_{z_{D}}^{z} D_{1}\left(z^{\prime}\right) d z^{\prime}}
$$

The asymmetry at $z>z_{D}$ then reads:

$$
\begin{aligned}
N_{\alpha \alpha}(z) & \simeq-\epsilon_{\alpha \alpha}^{(1)} \int_{z_{D}}^{z} \frac{d N_{N_{1}}}{d z^{\prime}} e^{-\int_{z^{\prime}}^{z} W_{1}\left(z^{\prime \prime}\right) p_{1 \alpha} d z^{\prime \prime}} d z^{\prime} \\
& \simeq-\epsilon_{\alpha \alpha}^{(1)} \int_{z_{D}}^{z} \frac{d N_{N_{1}}}{d z^{\prime}}\left(1-\int_{z^{\prime}}^{z} W_{1}\left(z^{\prime \prime}\right) p_{1 \alpha} d z^{\prime \prime}\right) d z^{\prime} \\
& \simeq \epsilon_{\alpha \alpha}^{(1)}\left(N_{N_{1}}^{e q}\left(z_{0}\right)-N_{N_{1}}(z)\right)-p_{1 \alpha} \epsilon_{\alpha \alpha}^{(1)} \int_{z_{D}}^{z} d z^{\prime} D_{1}\left(z^{\prime}\right) N_{N_{1}}\left(z^{\prime}\right) \int_{z^{\prime}}^{z} d z^{\prime \prime} W_{1}\left(z^{\prime \prime}\right) .
\end{aligned}
$$

The final asymmetry $B-L$ is then given by:

$$
N_{\alpha \alpha}(\infty)=\epsilon_{\alpha \alpha}^{(1)} N_{N_{1}}^{e q}\left(z_{0}\right)-\epsilon_{\alpha \alpha}^{(1)} p_{1 \alpha} \int_{z_{D}}^{z} d z^{\prime} D_{1}\left(z^{\prime}\right) N_{N_{1}}\left(z^{\prime}\right) \int_{z^{\prime}}^{z} d z^{\prime \prime} W_{1}\left(z^{\prime \prime}\right),
$$

which in the two-flavour approximation becomes

$$
N_{B-L}^{1 \mathrm{BE} 2 \mathrm{~F}}(\infty)=\epsilon^{(1)} N_{N_{1}}^{e q}\left(z_{0}\right)-\left(\epsilon_{\tau \tau}^{(1)} p_{1 \tau}+\epsilon_{\tau^{\perp} \tau^{\perp}}^{(1)} p_{1 \tau^{\perp}}\right) \mathcal{A}\left(\kappa_{1}\right)
$$

with

$$
\mathcal{A}\left(\kappa_{1}\right) \equiv \int_{z_{D}}^{\infty} d z^{\prime} D_{1}\left(z^{\prime}\right) N_{N_{1}}\left(z^{\prime}\right) \int_{z^{\prime}}^{z} d z^{\prime \prime} W_{1}\left(z^{\prime \prime}\right) d z^{\prime}
$$

Note that, if not for the second term in (B.20), when $\epsilon^{(1)}=0$ the final asymmetry would vanish.

\section{Approximate 1-to-2 flavour transitional mass scale}

In this appendix we present an analytical approximation for the mass scale of the 1-to- 2 flavour transition, $M_{10}$. In the following discussion we are not going to consider the effects of the $\mu$-Yukawas interactions, thus we set $\Lambda_{\mu}=\Gamma_{\mu} / H z=0$. We notice that what controls the scale of the 1-to-2 flavour transition is the coefficient that multiplies the integrated wash-out term in eq. (3.23), that is

$$
\mathcal{T}\left(M_{1}, p_{1 \tau}\right) \equiv \Lambda_{\tau} p_{1 \tau} p_{1 \tau^{\perp}}
$$

In the "standard" picture, we find that typically $p_{1 \tau} p_{1 \tau^{\perp}} \approx 0.25$ and the transition happens at a mass scale $M_{10} \approx 10^{12} \mathrm{GeV}$. We then define $\mathcal{T}_{12} \equiv \mathcal{T}\left(M_{1}=10^{12} \mathrm{GeV}, p_{1 \tau}=0.5\right)$. Our ansatz is that the condition

$$
\mathcal{T}\left(M_{10}, p_{1 \tau}\right) \approx \mathcal{T}_{12}
$$


gives approximately the mass scale of the 1-to-2 flavour transition. In terms of $M_{10}$ the above condition reads:

$$
\frac{M_{10}}{10^{12} \mathrm{GeV}} \approx 4 p_{1 \tau} p_{1 \tau^{\perp}} \text {. }
$$

For the values of the parameters used to obtain the bottom-left (right) panel of figure 6 , for example, we get $4 p_{1 \tau} p_{1 \tau^{\perp}} \cong 0.1\left(6 \times 10^{-3}\right)$, and correspondingly the mass scale of the transition is found at $M_{10} \cong 10^{11} \mathrm{GeV}\left(6 \times 10^{9} \mathrm{GeV}\right)$, in agreement with the figure. We find, however, that the above approximation can underestimate $M_{10}$ by a factor $\mathcal{O}(1.5-2.5)$ when $M_{10} \approx 10^{12} \mathrm{GeV}$. The approximation is more accurate when $M_{10} \ll 10^{12} \mathrm{GeV}$, as in the "non-standard" scenarios discussed in the present paper.

Open Access. This article is distributed under the terms of the Creative Commons Attribution License (CC-BY 4.0), which permits any use, distribution and reproduction in any medium, provided the original author(s) and source are credited.

\section{References}

[1] M. Fukugita and T. Yanagida, Baryogenesis Without Grand Unification, Phys. Lett. B 174 (1986) 45 [INSPIRE].

[2] V.A. Kuzmin, V.A. Rubakov and M.E. Shaposhnikov, On the Anomalous Electroweak Baryon Number Nonconservation in the Early Universe, Phys. Lett. B 155 (1985) 36 [INSPIRE].

[3] D. Bödeker and W. Buchmüller, Baryogenesis from the weak scale to the grand unification scale, Rev. Mod. Phys. 93 (2021) 035004 [arXiv:2009.07294] [INSPIRE].

[4] P. Minkowski, $\mu \rightarrow$ er at a Rate of One Out of $10^{9}$ Muon Decays?, Phys. Lett. B 67 (1977) 421 [INSPIRE].

[5] T. Yanagida, Horizontal gauge symmetry and masses of neutrinos, Conf. Proc. C $\mathbf{7 9 0 2 1 3 1}$ (1979) 95 [INSPIRE].

[6] M. Gell-Mann, P. Ramond and R. Slansky, Complex Spinors and Unified Theories, Conf. Proc. C 790927 (1979) 315 [arXiv: 1306.4669] [InSPIRE].

[7] S.L. Glashow, The Future of Elementary Particle Physics, NATO Sci. Ser. B 61 (1980) 687 [INSPIRE].

[8] R.N. Mohapatra and G. Senjanović, Neutrino Mass and Spontaneous Parity Nonconservation, Phys. Rev. Lett. 44 (1980) 912 [INSPIRE].

[9] A.D. Sakharov, Violation of CP Invariance, $C$ asymmetry, and baryon asymmetry of the universe, Pisma Zh. Eksp. Teor. Fiz. 5 (1967) 32.

[10] S. Davidson and A. Ibarra, A Lower bound on the right-handed neutrino mass from leptogenesis, Phys. Lett. B 535 (2002) 25 [hep-ph/0202239] [INSPIRE].

[11] E. Nardi, Y. Nir, E. Roulet and J. Racker, The Importance of flavor in leptogenesis, JHEP 01 (2006) 164 [hep-ph/0601084] [INSPIRE].

[12] A. Abada, S. Davidson, F.-X. Josse-Michaux, M. Losada and A. Riotto, Flavor issues in leptogenesis, JCAP 04 (2006) 004 [hep-ph/0601083] [INSPIRE].

[13] A. Abada, S. Davidson, A. Ibarra, F.X. Josse-Michaux, M. Losada and A. Riotto, Flavour Matters in Leptogenesis, JHEP 09 (2006) 010 [hep-ph/0605281] [INSPIRE]. 
[14] R. Barbieri, P. Creminelli, A. Strumia and N. Tetradis, Baryogenesis through leptogenesis, Nucl. Phys. B 575 (2000) 61 [hep-ph/9911315] [INSPIRE].

[15] H.B. Nielsen and Y. Takanishi, Baryogenesis via lepton number violation and family replicated gauge group, Nucl. Phys. B 636 (2002) 305 [hep-ph/0204027] [InSPIRE].

[16] T. Endoh, T. Morozumi and Z.-h. Xiong, Primordial lepton family asymmetries in seesaw model, Prog. Theor. Phys. 111 (2004) 123 [hep-ph/0308276] [INSPIRE].

[17] S. Pascoli, S.T. Petcov and A. Riotto, Connecting low energy leptonic CP-violation to leptogenesis, Phys. Rev. D 75 (2007) 083511 [hep-ph/0609125] [INSPIRE].

[18] S. Pascoli, S.T. Petcov and A. Riotto, Leptogenesis and Low Energy CP-violation in Neutrino Physics, Nucl. Phys. B 774 (2007) 1 [hep-ph/0611338] [INSPIRE].

[19] S. Blanchet and P. Di Bari, Flavor effects on leptogenesis predictions, JCAP 03 (2007) 018 [hep-ph/0607330] [INSPIRE].

[20] G.C. Branco, R. Gonzalez Felipe and F.R. Joaquim, A New bridge between leptonic CP-violation and leptogenesis, Phys. Lett. B 645 (2007) 432 [hep-ph/0609297] [INSPIRE].

[21] A. Anisimov, S. Blanchet and P. Di Bari, Viability of Dirac phase leptogenesis, JCAP 04 (2008) 033 [arXiv:0707.3024] [INSPIRE].

[22] E. Molinaro and S.T. Petcov, The Interplay Between the 'Low' and 'High' Energy CP-Violation in Leptogenesis, Eur. Phys. J. C 61 (2009) 93 [arXiv:0803.4120] [InSPIRE].

[23] E. Molinaro and S.T. Petcov, A Case of Subdominant/Suppressed 'High Energy' Contribution to the Baryon Asymmetry of the Universe in Flavoured Leptogenesis, Phys. Lett. $B 671$ (2009) 60 [arXiv:0808.3534] [INSPIRE].

[24] M.J. Dolan, T.P. Dutka and R.R. Volkas, Dirac-Phase Thermal Leptogenesis in the extended Type-I Seesaw Model, JCAP 06 (2018) 012 [arXiv: 1802.08373] [INSPIRE].

[25] K. Moffat, S. Pascoli, S.T. Petcov, H. Schulz and J. Turner, Three-flavored nonresonant leptogenesis at intermediate scales, Phys. Rev. D 98 (2018) 015036 [arXiv:1804.05066] [INSPIRE].

[26] K. Moffat, S. Pascoli, S.T. Petcov and J. Turner, Leptogenesis from Low Energy CP Violation, JHEP 03 (2019) 034 [arXiv: 1809.08251] [INSPIRE].

[27] I. Brivio, K. Moffat, S. Pascoli, S.T. Petcov and J. Turner, Leptogenesis in the Neutrino Option, JHEP 10 (2019) 059 [Erratum ibid. 02 (2020) 148] [arXiv:1905.12642] [INSPIRE].

[28] I. Brivio and M. Trott, Radiatively Generating the Higgs Potential and Electroweak Scale via the Seesaw Mechanism, Phys. Rev. Lett. 119 (2017) 141801 [arXiv:1703.10924] [InSPIRE].

[29] A. De Simone and A. Riotto, On the impact of flavour oscillations in leptogenesis, JCAP 02 (2007) 005 [hep-ph/0611357] [INSPIRE].

[30] S. Blanchet, P. Di Bari and G.G. Raffelt, Quantum Zeno effect and the impact of flavor in leptogenesis, JCAP 03 (2007) 012 [hep-ph/0611337] [INSPIRE].

[31] S. Blanchet, P. Di Bari, D.A. Jones and L. Marzola, Leptogenesis with heavy neutrino flavours: from density matrix to Boltzmann equations, JCAP 01 (2013) 041 [arXiv: 1112.4528] [INSPIRE].

[32] Particle Data Group collaboration, Review of Particle Physics, Phys. Rev. D 98 (2018) 030001 [INSPIRE]. 
[33] S.M. Bilenky, J. Hosek and S.T. Petcov, On Oscillations of Neutrinos with Dirac and Majorana Masses, Phys. Lett. B 94 (1980) 495 [INSPIRE].

[34] Particle Data Group collaboration, Review of Particle Physics, PTEP 2020 (2020) 083C01 [inSPIRE].

[35] F. Capozzi, E. Di Valentino, E. Lisi, A. Marrone, A. Melchiorri and A. Palazzo, Global constraints on absolute neutrino masses and their ordering, Phys. Rev. D 95 (2017) 096014 [Addendum ibid. 101 (2020) 116013] [arXiv:2003.08511] [InSPIRE].

[36] I. Esteban, M.C. Gonzalez-Garcia, M. Maltoni, T. Schwetz and A. Zhou, The fate of hints: updated global analysis of three-flavor neutrino oscillations, JHEP 09 (2020) 178 [arXiv: 2007.14792] [INSPIRE].

[37] T2K collaboration, Constraint on the matter-antimatter symmetry-violating phase in neutrino oscillations, Nature $\mathbf{5 8 0}$ (2020) 339 [Erratum ibid. $\mathbf{5 8 3}$ (2020) E16] [arXiv: 1910.03887] [INSPIRE].

[38] A. Himmel, New oscillation results from the NOvA experiment, talk given at the XXIX International Conference on Neutrino Physics and Astrophysics, June 22-July 2, Chicago, U.S.A. (2020), available on Zenodo.

[39] N. Cabibbo, Time Reversal Violation in Neutrino Oscillation, Phys. Lett. B 72 (1978) 333 [INSPIRE].

[40] V.D. Barger, K. Whisnant and R.J.N. Phillips, CP Violation in Three Neutrino Oscillations, Phys. Rev. Lett. 45 (1980) 2084 [INSPIRE].

[41] P.I. Krastev and S.T. Petcov, Resonance Amplification and $t$ Violation Effects in Three Neutrino Oscillations in the Earth, Phys. Lett. B 205 (1988) 84 [INSPIRE].

[42] C. Jarlskog, A Basis Independent Formulation of the Connection Between Quark Mass Matrices, CP-violation and Experiment, Z. Phys. C 29 (1985) 491 [InSPIRE].

[43] P. Langacker, S.T. Petcov, G. Steigman and S. Toshev, On the Mikheev-Smirnov-Wolfenstein (MSW) Mechanism of Amplification of Neutrino Oscillations in Matter, Nucl. Phys. B 282 (1987) 589 [INSPIRE].

[44] S.M. Bilenky and S.T. Petcov, Massive Neutrinos and Neutrino Oscillations, Rev. Mod. Phys. 59 (1987) 671 [Erratum ibid. 61 (1989) 169] [Erratum ibid. 60 (1988) 575] [INSPIRE].

[45] S.M. Bilenky, S. Pascoli and S.T. Petcov, Majorana neutrinos, neutrino mass spectrum, CP-violation and neutrinoless double beta decay. 1. The Three neutrino mixing case, Phys. Rev. D 64 (2001) 053010 [hep-ph/0102265] [INSPIRE].

[46] S.T. Petcov, Neutrinoless double beta decay searches: theory and motivation, talk given at the XXIX International Conference on Neutrino Physics and Astrophysics, June 22-July 2, Chicago, U.S.A. (2020), available on Zenodo.

[47] KamLAND-Zen collaboration, Search for Majorana Neutrinos near the Inverted Mass Hierarchy Region with KamLAND-Zen, Phys. Rev. Lett. 117 (2016) 082503 [Addendum ibid. 117 (2016) 109903] [arXiv: 1605.02889] [INSPIRE].

[48] GERDA collaboration, Final Results of GERDA on the Search for Neutrinoless Double- $\beta$ Decay, Phys. Rev. Lett. 125 (2020) 252502 [arXiv:2009. 06079] [INSPIRE].

[49] J.T. Penedo and S.T. Petcov, The $10^{-3} \mathrm{eV}$ frontier in neutrinoless double beta decay, Phys. Lett. B $\mathbf{7 8 6}$ (2018) 410 [arXiv:1806.03203] [INSPIRE]. 
[50] KATRIN collaboration, Improved Upper Limit on the Neutrino Mass from a Direct Kinematic Method by KATRIN, Phys. Rev. Lett. 123 (2019) 221802 [arXiv:1909.06048] [INSPIRE].

[51] M. Aker et al., First direct neutrino-mass measurement with sub-eV sensitivity, arXiv:2105.08533 [INSPIRE].

[52] Particle Data Group collaboration, Review of Particle Physics, PTEP 2020 (2020) 083C01 [INSPIRE].

[53] J. Lopez-Pavon, E. Molinaro and S.T. Petcov, Radiative Corrections to Light Neutrino Masses in Low Scale Type I Seesaw Scenarios and Neutrinoless Double Beta Decay, JHEP 11 (2015) 030 [arXiv: 1506.05296] [inSPIRE].

[54] A. Pilaftsis, Radiatively induced neutrino masses and large Higgs neutrino couplings in the standard model with Majorana fields, Z. Phys. C 55 (1992) 275 [hep-ph/9901206] [InSPIRE].

[55] D. Aristizabal Sierra and C.E. Yaguna, On the importance of the 1-loop finite corrections to seesaw neutrino masses, JHEP 08 (2011) 013 [arXiv:1106.3587] [INSPIRE].

[56] J. Lopez-Pavon, S. Pascoli and C.-f. Wong, Can heavy neutrinos dominate neutrinoless double beta decay?, Phys. Rev. D 87 (2013) 093007 [arXiv:1209.5342] [INSPIRE].

[57] W. Grimus and L. Lavoura, One-loop corrections to the seesaw mechanism in the multi-Higgs-doublet standard model, Phys. Lett. B 546 (2002) 86 [hep-ph/0207229] [INSPIRE].

[58] J.A. Casas and A. Ibarra, Oscillating neutrinos and $\mu \rightarrow e, \gamma$, Nucl. Phys. B 618 (2001) 171 [hep-ph/0103065] [INSPIRE].

[59] A.S. Adam, Y. Kawamura and T. Morozumi, A model with light and heavy scalars in view of the effective theory, arXiv:2108.03639 [INSPIRE].

[60] R.J. Cooke, M. Pettini and C.C. Steidel, One Percent Determination of the Primordial Deuterium Abundance, Astrophys. J. 855 (2018) 102 [arXiv:1710.11129] [INSPIRE].

[61] PLANCK collaboration, Planck 2018 results. VI. Cosmological parameters, Astron. Astrophys. 641 (2020) A6 [Erratum ibid. 652 (2021) C4] [arXiv: 1807. 06209] [INSPIRE].

[62] P.S.B. Dev, P. Di Bari, B. Garbrecht, S. Lavignac, P. Millington and D. Teresi, Flavor effects in leptogenesis, Int. J. Mod. Phys. A 33 (2018) 1842001 [arXiv: 1711.02861] [InSPIRE].

[63] S. Davidson, E. Nardi and Y. Nir, Leptogenesis, Phys. Rept. 466 (2008) 105.

[64] W. Buchmüller, P. Di Bari and M. Plümacher, Leptogenesis for pedestrians, Annals Phys. 315 (2005) 305 [hep-ph/0401240] [INSPIRE].

[65] L. Covi, E. Roulet and F. Vissani, CP violating decays in leptogenesis scenarios, Phys. Lett. B 384 (1996) 169 [hep-ph/9605319] [InSPIRE].

[66] L. Covi and E. Roulet, Baryogenesis from mixed particle decays, Phys. Lett. B 399 (1997) 113 [hep-ph/9611425] [INSPIRE].

[67] W. Buchmüller and M. Plümacher, CP asymmetry in Majorana neutrino decays, Phys. Lett. B 431 (1998) 354 [hep-ph/9710460] [INSPIRE].

[68] S. Biondini et al., Status of rates and rate equations for thermal leptogenesis, Int. J. Mod. Phys. A 33 (2018) 1842004 [arXiv: 1711.02864] [INSPIRE]. 
[69] A. Granelli, K. Moffat, Y.F. Perez-Gonzalez, H. Schulz and J. Turner, ULYSSES: Universal LeptogeneSiS Equation Solver, Comput. Phys. Commun. 262 (2021) 107813 [arXiv: 2007.09150] [INSPIRE].

[70] C.S. Fong and J. Racker, On fast CP-violating interactions in leptogenesis, JCAP 07 (2010) 001 [arXiv: 1004.2546] [INSPIRE].

[71] Z.-z. Xing and D. Zhang, A direct link between unflavored leptogenesis and low-energy CP-violation via the one-loop quantum corrections, JHEP 04 (2020) 179 [arXiv: 2003.00480] [INSPIRE].

[72] C.S. Fong, E. Nardi and A. Riotto, Leptogenesis in the Universe, Adv. High Energy Phys. $2012(2012) 158303$ [arXiv:1301.3062] [INSPIRE]. 